\title{
ScRNA-seq reveals tumor microenvironment remodeling induced by local intervention-based immunotherapy
}

Ashley R. Hoover ${ }^{1,2}$, Kaili Liu ${ }^{1 \ddagger}$, Christa I. DeVette ${ }^{3}$, Jason R. Krawic ${ }^{3}$, Connor L. West ${ }^{1}$, Delaney Medcalf ${ }^{2}$, Alana L. Welm ${ }^{4}$, Xiao-Hong Sun ${ }^{2}$, William H. Hildebrand ${ }^{3}$, and Wei R. Chen $^{1 *}$

${ }^{1}$ Stephenson School of Biomedical Engineering, University of Oklahoma, Norman, OK, USA

${ }^{2}$ Arthritis \& Clinical Immunology Research Program, Oklahoma Medical Research Foundation, Oklahoma City, OK, USA

${ }^{3}$ Department of Microbiology and Immunology, University of Oklahoma Health Sciences Center, Oklahoma City, OK, USA

${ }^{4}$ Huntsman Cancer Institute, University of Utah, Salt Lake City, UT, USA

‡ Authors contributed equally

* Corresponding author:Wei-R-Chen@ou.edu 


\section{ABSTRACT}

Laser immunotherapy (LIT) combines local photothermal therapy (PTT), to disrupt tumor homeostasis and release tumor antigens, and an intratumorally administered immunostimulant, N-dihydrogalactochitosan (GC), to induce antitumor immune responses. We performed single-cell RNA sequencing on tumor-infiltrating leukocytes of MMTV-PyMT mouse mammary tumors to characterize LIT-induced myeloid and lymphoid compartment remodeling. Analysis of 49,380 single cell transcriptomes from different treatment groups revealed that proinflammatory IFN $\alpha$, IFN $\gamma$, and TNF $\alpha$ cytokine signaling pathways were enriched in both lymphoid and myeloid cells isolated from LIT-treated tumors. The $\mathrm{CD} 4^{+}$and $\mathrm{CD}^{+} \mathrm{T}$ cells in LIT treated tumors resided in an activated state while immune cells in untreated and PTT-treated tumors remained in a neutral/resting state. Additionally, monocytes recruited into the LIT-treated tumors were driven towards proinflammatory M1-like macrophage phenotypes or monocyte-derived dendritic cells. Our results reveal that LIT prompts immunological remodeling of the tumor microenvironment by initiating broad proinflammatory responses to drive antitumor immunity.

\section{STATEMENT OF SIGNIFICANCE}

Transcriptome profiling of tumor infiltrating leukocytes revealed that localized laser immunotherapy (LIT) greatly enhanced antitumor $\mathrm{T}$ cell activity by promoting proinflammatory myeloid cell responses within the tumor microenvironment. This manuscript demonstrates that LIT broadly stimulates antitumor immunity and has great potential to synergize with current immunotherapies to increase their efficacy. 


\section{INTRODUCTION}

A major challenge in cancer treatment is the failure of the host immune system to detect and destroy cancer cells (1), particularly metastasis, which causes $90 \%$ of cancerrelated deaths. Effective therapies for metastatic cancers remain elusive (2). Cancer immunotherapy, which boosts the immune response to cancer, holds high promise for treating metastatic cancers (3). Various immunotherapies, such as cytokine and monoclonal antibody therapy $(4,5)$ adoptive cell therapy (6), chimeric antigen receptor T-cell (CAR-T) therapy (7), tumor vaccines (8), and immune checkpoint therapy (ICT) (9), represent potent anti-cancer treatments. Unfortunately, most of these treatments rely on existing antitumor immune responses or prerequisite knowledge of target tumor antigens. Since most cancer patients have poor antitumor immunity, efficacy and response rates of current immunotherapies are low across various tumors in clinical studies $(10,11)$.

A local intervention-based approach that induces systemic, long-term antitumor immunity represents an ideal therapy for metastatic cancers. Laser immunotherapy (LIT) was developed to achieve this objective. LIT has two components: 1) local photothermal therapy (PTT) for disrupting target tumor homeostasis and releasing tumor antigens $(12,13)$, and 2) local administration of an immunostimulant, such as $\mathrm{N}$ dihydrogalactochitosan (GC) $(14,15)$. In preliminary clinical studies for patients with metastatic, treatment-recalcitrant breast cancer and melanoma, LIT successfully reduced and/or eliminated the treated primary tumors and untreated metastases in the lungs (16-18). Because of these promising outcomes in our pre-clinical and preliminary clinical studies, we are characterizing the underlying mechanism whereby LIT achieves powerful antitumor effects. Here, we hypothesized that LIT activates both innate and adaptive immune responses to foster sustained antitumor immunity.

To test this hypothesis, we evaluated the effects of LIT on breast tumors arising from the mouse mammary tumor virus-polyoma middle $T$ (MMTV-PyMT) transgenic mice. MMTV-PyMT tumors have high penetrance and emulate the histological stages of human luminal type $B$ breast cancer, providing an excellent immune competent model for studying the tumor microenvironment (TME) $(19,20)$. Single-cell RNA sequencing 
(scRNA-seq) on human breast tumors revealed that tumor associated macrophages (TAMs), monocytes, and other myeloid and lymphoid cells are diverse in the TME (21). Therefore, we performed scRNA-seq on MMTV-PyMT tumors, to capture the complexity and heterogeneity of the TME following LIT treatment.

Using a combination of flow cytometry, T cell depletion, and scRNA-seq analysis, we revealed that LIT drives a global shift in immune cells towards various inflammatory states, fostering prolonged antitumor immunity. To evaluate LIT activation on the tumorinfiltrating leukocytes (TILs) in the TME, we analyzed scRNA-seq data on TILs from untreated (CTRL), PTT, GC, and LIT (PTT+GC) treated MMTV-PyMT tumors. Our results revealed that changes in the myeloid compartment potentiated adaptive immunity by increasing the activation of tumor-resident naïve, effector, and memory $\mathrm{CD}^{+}$and $\mathrm{CD} 8^{+} \mathrm{T}$ cells. LIT also induced a proinflammatory response in all the innate immune cells within the TME, increasing M1-like macrophages and prompting proinflammatory signals in M2-like macrophages. Together, our results demonstrate that LIT initiates broad changes within the TME, especially antitumor immune responses in both myeloid and lymphoid compartments, ultimately leading to systemic antitumor immunity. 


\section{RESULTS}

\section{LIT extends the survival of mice bearing MMTV-PyMT breast tumors.}

The therapeutic effects of LIT on breast tumors were determined using the MMTVPyMT transgenic tumor model. Spontaneous MMTV-PyMT tumor cells were isolated from MMTV-PyMT transgenic mice as previously described (22) and were injected into the mammary fat pad of wild type female FVB mice (Figure 1A). When tumors reached a size of $0.5 \mathrm{~cm}^{3}$, mice were divided into four treatment groups: Untreated controls (CTRL), PTT alone, GC alone, and LIT (PTT+GC). Treated animals were monitored up to 60 days following treatment, and euthanized once tumors reached ethical endpoints. LIT significantly reduced tumor growth and increased the survival rate compared to other groups (Figure 1B-C).

Since cytotoxic T cells are crucial to antitumor immune responses, we evaluated the extent of cytotoxic T cell induction by LIT in the TME 10 days after treatment by flow cytometry. Thermal effects of laser irradiation reduce tumor size, resulting in significantly smaller tumors in the PTT and LIT groups than that in CTRL and GC groups (Figure S1A). For comparison, TIL cellularity was divided by tumor volume. The total normalized $\mathrm{CD}_{4} 5^{+}$cellularity $/ \mathrm{mm}^{3}$ (Figure $\mathrm{S} 1 \mathrm{~A}$ ), following the gating strategies shown in Figure S1B, showed no significant differences among different treatment groups. The effector cells $\left(\mathrm{CD} 45^{+} \mathrm{CD} 3^{+} \mathrm{CD} 8^{+} \mathrm{CD} 44^{+}\right)$and memory $\mathrm{T}$ cells $\left(\mathrm{CD} 45^{+} \mathrm{CD}^{+} \mathrm{CD}^{+} \mathrm{CD} 44^{\mathrm{High}} \mathrm{CD} 2 \mathrm{~L}^{+}\right)$in $\mathrm{LIT}$ and $\mathrm{GC}$ groups had a trending increase, while the $\mathrm{CD}_{4}{ }^{+} \mathrm{CD} 3^{+} \mathrm{CD} 8{ }^{+} \mathrm{CD} 44^{\mathrm{Low}} \mathrm{CD} 6 \mathrm{~L}^{+}$and $\mathrm{CD} 45^{+} \mathrm{CD}^{+} \mathrm{CD}^{+} \mathrm{CD} 2 \mathrm{~L}^{+}$naïve $\mathrm{T}$ cells in LIT and GC groups were similar to that of CTRL and PTT groups (Figure S1A).

To further evaluate $\mathrm{CD}^{+} \mathrm{T}$ cell populations, using the gating strategy in Figure S1B, t-distributed Stochastic Neighbor Embedding (t-SNE) plots were generated from 3 concatenated samples from each group using FlowJo (Figure S1C). These plots revealed more diversity within the $\mathrm{CD}^{+} \mathrm{T}$ cells from LIT-treated tumors based on their distribution and number of distinct clusters (Figure 1D). For example, the $\mathrm{CD}^{2} \mathrm{~L}^{+}$cells in the LIT-treated tumors separated into two distinct groups (blue clusters, red arrows). The effector cells (red clusters, black arrows) from the LIT-treated tumors also separated into distinct clusters, with a much greater distance between them on the 
SNE plot, again suggesting more $\mathrm{T}$ cell diversity. There was also a large reduction in the proportion of $\mathrm{CD}^{2} 8^{+} \mathrm{CD} 69^{-}$cells (Figure 1D, black clusters, red arrows) and an increase in the $\mathrm{CD}_{2}{ }^{+} \mathrm{CD}^{+} 9^{+}$cells in the LIT-treated tumors, suggesting the differentiation and activation of $\mathrm{CD} 8^{+} \mathrm{T}$ cells following LIT. The intensity and distribution of $\mathrm{CD} 9^{+}, \mathrm{CD}^{2} 8^{+}$, and $\mathrm{CD} 103^{+}$expression on $\mathrm{CD}^{+} \mathrm{T}$ cells were all increased in the LIT-treated tumors (Figure S1C). The mean expression levels of CD69, as determined by mean fluorescence intensity (MFI), on the $\mathrm{CD} 3^{+} \mathrm{CD} 8^{+} \mathrm{CD} 44^{+}$and

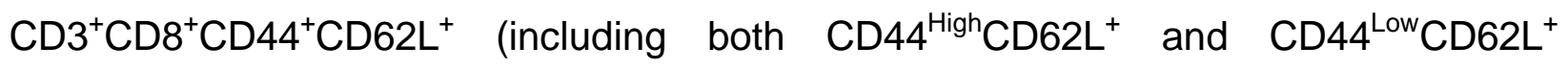
populations) $T$ cell subsets, were statistically elevated in the LIT-treated tumors compared to GC alone, and trending up compared to CTRL and PTT (Figure 1E). CD28 was also significantly increased on the $\mathrm{CD} 3^{+} \mathrm{CD} 8^{+} \mathrm{CD} 44^{+}$cells and trending up on the $\mathrm{CD}^{+} \mathrm{CD} 8{ }^{+} \mathrm{CD} 44^{+} \mathrm{CD} 62 \mathrm{~L}^{+} \mathrm{T}$ cell subsets (Figure $1 \mathrm{E}$ ). These results indicate that the $\mathrm{CD}^{+} \mathrm{T}$ cells are activated in response to LIT, but this analysis did not fully explain the unique effects of LIT on tumor growth inhibition.

We next investigated the contributions of $\mathrm{CD}^{+}$and/or $\mathrm{CD}^{+} \mathrm{T}$ cells to the therapeutic effect of LIT, using depletion antibodies (Figure S1D), with $>95 \%$ depletion confirmed by flow cytometry. Loss of $\mathrm{CD}^{+} \mathrm{T}$ cells after LIT treatment abrogated the therapeutic benefits of LIT, suggesting that cytotoxic T lymphocytes (CTLs) are critical for downstream responses following LIT (Figure 1F-G). Surprisingly, loss of CD4 $4^{+} \mathrm{T}$ cells following LIT treatment delayed tumor growth and prolonged survival in a manner superior to LIT alone (Figure $1 \mathrm{G}$ ). These findings suggest that $\mathrm{CD}^{+} \mathrm{T}$ cells are imperative to mounting an anti-tumor response, but $\mathrm{CD}^{+} \mathrm{T}$ cells may serve as an avenue for immune inhibition post-LIT exposure in this model. We therefore proceeded with scRNA-seq to provide a transcriptomic explanation for the immunological changes driven by LIT.

\section{ScRNA-seq reveals diversity of tumor-infiltrating leukocytes induced by LIT}

To investigate a broad spectrum of immune cell alterations in the TME, scRNA-seq was performed, following the workflow depicted in Figure S1E. Mice bearing MMTV-PyMT tumors were divided into four treatment groups ( $n=4$ per group). Nine days after treatment, tumor-infiltrating leukocytes (TILs) were pooled from mice in each group and 
enriched. First, all 49,380 TILs (11,584 CTRL; 14,071 PTT; 12,501 GC; 11,224 LIT) were integrated for scRNA-seq. Unsupervised clustering analysis was performed using Seurat $R$ package (23). Using the default shared-nearest neighbor (SNN) resolution (0.5), t-SNE and Uniform Manifold Approximation and Projection (UMAP) plots unveiled 20 (0-19) distinct cell clusters, representative of both myeloid and lymphoid cell lineages (Figure 2A-B). The unsupervised analysis of the top 10 differentially expressed genes (DEGs) from each cell confirmed that the TILs were successfully delineated into 20 distinct clusters as visualized by the heatmap (Figure $2 \mathrm{C}$ ).

To accurately annotate the immune cell clusters, we used a combination of the cluster identity predictor (CIPR) (https://aekiz.shinyapps.io/CIPR/) which is linked to the Immunological Genome Project (ImmGen) and a publicly available cell specific gene list (24). With the top 5 scores generated by CIPR, we identified the following TIL populations: T cells, Yס-T cells, Tregs, B cells, pre-B cells, granulocytes, monocytes, macrophages $(\mathrm{M} \varphi)$, dendritic cells (DCs), and natural killer (NK) cells (Figure S2A). Stromal cell and B cell clusters (15 and 19) were excluded from the rest of the analysis. With CIPR, cells in cluster 6 cannot be clearly distinguished as either T or B cells by the top 5 scores. To overcome this limitation, we relied on a series of commonly used immune cell genes to distinguish and confirm the cell type in each cluster (Figure 2D). The dot plots show the average expression levels and percentages of specific genes commonly used to identify immune cell subtypes (Figure 2D). The specificity of these selected genes is illustrated with the correlation plot for the entire cell-derived gene expression patterns (Figure S2B), indicating that their expressions correlate with and represent specific cell clusters.

By combining the gene expression and cluster analysis we annotated all cell clusters (Figure 2A-D). For example, cells in cluster 6 were activated, proliferating CD8 ${ }^{+}$ $\mathrm{T}$ cells, with high expression of Cd8a, Cd8b1, Gzmb, IFNg, and cell cycling marker Mki67. The combination of unsupervised clustering using high throughput signature annotation and common traditional immune cell genes for validation were mutually supportive and complementary (Figures 2C-D, Figures S2A-B).

Separating the UMAP of integrated cells (Figure 2B) into four individual plots (Figure 2E), we discovered that several clusters were significantly different in the LIT- 
treated tumors compared to that of CTRL and PTT groups (Figure 2E, red arrows). We also calculated the immune cell proportions in individual compartments (Figure 2F-G) and the proportions of immune cells in individual clusters against total TILs (Figure S2C). Specifically, LIT increased the proportions of activated CD8 ${ }^{+} \mathrm{T}$ cells (cluster 1) and memory CD4 ${ }^{+} \mathrm{T}$ cells (cluster 0 ), and decreased cytotoxic $\mathrm{\gamma} \delta-\mathrm{T}$ cells (cluster 3 ) (Figure 2F). As shown in Figure 2G, LIT increased the proportions of granulocytes (cluster 10) and decreased $\mathrm{M} \varphi$ (cluster 4). These findings support our hypothesis that LIT induces broad changes to TILs in the TME.

\section{LIT alters lymphoid cell composition and gene expression in the TME}

We next explored transcriptomic changes within specific $\mathrm{T}$ cell subsets, since diversity of $T$ cell subtypes in the TME have profound effects on antitumor immunity. Our scRNAseq data demonstrated that activated $\mathrm{CD}^{+} \mathrm{T}$ cells and memory $\mathrm{CD}^{+} \mathrm{T}$ cells were significantly increased in LIT-treated tumors (Figure 2F). To further analyze these cell types, we performed higher resolution re-clustering of the lymphoid cells, excluding $B$ cells, and obtained 21 clusters (clusters 0-20), as shown in Figure 3A. Expressions of common lymphoid cell markers, including Cd3e, Cd4, Cd8b1, Foxp3, Mki67, and Ncr1, were examined and mapped to cell clusters using UMAP plots (Figure S3A), and annotation was performed (Figure S3B). The increased resolution revealed the subtypes of lymphoid cells that experienced significant changes (Figure S3C, red arrows) with the activated $\mathrm{CD}^{+} \mathrm{T}$ cells from cluster 1 in Figure $2 \mathrm{~F}$ becoming clusters 0 and 3 in Figure 3B. LIT significantly upregulated naïve $\mathrm{CD}^{+} \mathrm{T}$ cells (cluster 0 ) and central-memory CD4 ${ }^{+} \mathrm{T}$ cells (cluster 9 ), as shown in Figure 3B. Clusters 5 and 6 both contained naïve CD4 ${ }^{+}$T cells, with cluster 5 resembling stimulated T cells and cluster 6 unstimulated (Figure 3B). Additionally, LIT decreased the cell ratio of cluster 1 (cytotoxic Yס-T cells) and induced a small proportion of $\mathrm{IL}-17^{+} \mathrm{IFNy}^{+} \mathrm{CD}^{+} \mathrm{T}$ cells (cluster 19; Figure 3B). The appearance of naïve T cells suggests that LIT can actively recruit these cells to the TME and potentially generate de novo $\mathrm{T}$ cell responses, not just enhance existing $\mathrm{T}$ cells responses. This is significant as LIT has the potential to generate better, more diverse $\mathrm{T}$ cell pool that limits exhaustion and immune evasion. 
To determine how the lymphoid cells are activated, we performed gene set enrichment analysis (GSEA). Using differentially expressed genes (DEGs), we focused on the comparison between LIT and CTRL groups. As shown in Figures 3C and S3D-E, LIT significantly upregulated IFN $\alpha$ and IFNy signaling pathways of activated $ү \bar{\delta}-\mathrm{T}$ cells (cluster 2), naïve CD8 ${ }^{+}$T cells (cluster 0 ), NK cells (cluster 4 ), the combined naïve CD4 ${ }^{+}$ T cells (cluster 5 and 6), CD4 ${ }^{+}$Tregs (cluster 11), and central memory CD4 ${ }^{+} \mathrm{T}$ cells (cluster 9). mTORC1, G2M checkpoint, and Myc targets pathways were downregulated by LIT in these cell clusters (Figure 3C and S3D-E). The signaling pathway analysis demonstrates that LIT promotes the antitumor type I/II IFNs and TNF pathways across different lymphoid cell types.

To find the common DEGs in five separate lymphoid cell clusters from different comparison groups, we generated Venn diagrams (Figure 3D). In these selected lymphoid subtypes, 65, 16, and 5 commonly shared DEGs were found in LIT vs CTRL, LIT vs PTT, and LIT vs GC, respectively (Figure 3D, Table S1). We also generated violin plots for a series of conserved DEGs for LIT vs CTRL in these selected T cell types and found that LIT significantly promoted the expression of several genes involved in T cell activation, function, and survival (Figure 3E and S3F and Table S1). For example, LIT promoted the expression of Gimap proteins, Dusp1 (MKP-1), Isg15, and Ptprc, which are involved in T cell survival, activation/function, and death (Figure S3F) (25-28). LIT also induced the expression of Fos, Jun, Ptprcap, and Lcp1 (Figure 3E), which are associated with T cell receptor (TCR) signaling (29-31), indicating that $T$ cells in LIT-treated tumors are responding to their cognate antigens. LIT also initiated changes in several other genes with less known functions in T cells, as listed in Table S1. In summary, our data reveal that LIT induces TCR signaling within T cells.

\section{LIT promotes naïve and memory $T$ cell activation in the TME}

To confirm that T cells in LIT-treated tumors were activated, we performed cell trajectory inference (CTI) analysis, using $R$ package monocle2 (32), on $\mathrm{CD}^{+}$(Figure 4A-D, S4A) and $\mathrm{CD}^{+} \mathrm{T}$ cells (Figure 4E-H and S4D). The $\mathrm{CD}^{+} \mathrm{T}$ cells were divided into 9 states (Figure S4A) with the naïve $\mathrm{CD}^{+} \mathrm{T}$ cells distributed on the right branches, including states 4, 5, and 6 (Figure 4A and S4A). Non-naïve subtypes of $\mathrm{CD}^{+} \mathrm{T}$ cells were 
distributed on the left branches, including states 1, 2, 3, 7, 8, and 9 (Figure 4A and S4A). When separated based on different treatments, the cell trajectory patterns were shifted by GC and LIT from upper states $(1,2,3,5,9)$ to lower states $(6,7,8)$, as shown in Figure 4B. To determine if the shift was indeed due to $\mathrm{T}$ cell activation, we generated volcano plots of the top DEGs of $\mathrm{CD}^{+} \mathrm{T}$ cells between LIT-treated and CTRL tumors (Figure S4B). The genes highly regulated in $\mathrm{CD}^{+} \mathrm{T}$ cells from LIT-treated tumors were associated with $\mathrm{T}$ cell activation (Figure S4B). Thus, we infer that cells in the upper branches are in a resting/neutral state (blue circle) and the cells in lower branches are in an activated state (red circles), as shown in Figure 4C-D. By examining individual $\mathrm{CD}^{+} \mathrm{T}$ cell clusters, we observed a shift in the CD8 ${ }^{+}$T cells in clusters $0,3,13$, and 16 towards the activated states in GC and LIT-treated tumors (Figure 4D). Overall, the proportion of $\mathrm{CD}^{+} \mathrm{T}$ cells in the activated state increased dramatically in the LITtreated tumors (Figure S4C).

We also performed CTI analysis on $\mathrm{CD}^{+} \mathrm{T}$ cells (Figure 4E and S4D). CD4 ${ }^{+} \mathrm{T}$ cells were divided into 11 states (Figure S4D). Naïve and central memory CD4 ${ }^{+} \mathrm{T}_{\text {cells }}$ were distributed on the right branches, which included states 6 through 11 (Figure 4E and S4D). Exhausted $\mathrm{CD}^{+} \mathrm{T}$ cells showed ubiquitous expression throughout the trajectory. The Tregs were localized at the left branches, in an opposite pattern to that of naïve $\mathrm{CD}^{+} \mathrm{T}$ cells (Figure 4E). When separated based on treatment groups, the cell trajectory patterns were shifted by GC and LIT from the upper states to lower states (Figure 4F), similar to that of $\mathrm{CD}^{+} \mathrm{T}$ cells (Figure 4B). To confirm this switch was also due to $\mathrm{T}$ cell activation, volcano plots from DEGs of $\mathrm{CD}^{+} \mathrm{T}$ cells between LIT-treated and CTRL tumors were generated (Figure S4E). The genes associated with $\mathrm{T}$ cell activation were upregulated in LIT-treated tumors (Figure S4E). Based on the gene expression data, we labeled the cells residing in the lower states (red circle) as activated and cells residing in the upper states (blue circles) as resting/neutral (Figure 4G-H). The proportion of cells residing in the resting/neutral state versus the activated state was significantly different in the GC and LIT-treated tumors compared to that in the CTRL and PTT-treated tumors (Figure S4F).

GC and LIT shifted CD4 ${ }^{+}$T cells towards the activated state in clusters 5, 6, 9, and 10 (Figure $4 \mathrm{H})$. Using our re-clustering analysis of the lymphoid cells, we noted that 
even though clusters 5 and 6 contained both naïve CD4 ${ }^{+}$T cells, the cells in cluster 5 were activated while cells in cluster 6 were resting/neutral. This likely explains the dramatic shift in these two populations by LIT (Figure 3B) as nearly all the T cells in cluster 5 reside in the activated state along with very few T cells from cluster 6 in the LIT-treated tumors (Figure 4H). Interestingly, only in the LIT-treated tumors we observed that all the central memory and exhausted T cells (cluster 9) resided in the activated state (Figure $4 \mathrm{H}$ ).

To determine how LIT is globally effecting $\mathrm{CD}^{+}$and $\mathrm{CD}^{+} \mathrm{T}$ cell activation, we performed GSEA on total CD8 ${ }^{+}$or $\mathrm{CD}^{+} \mathrm{T}$ cells from LIT-treated and CTRL tumors. LIT significantly upregulated proinflammatory signaling pathways IFNa, IFNy, and TNFa and downregulated Myc targets and G2M checkpoint regulators in both CD8 $^{+}$(Figure 4I) and $\mathrm{CD}^{+}$(Figure 4J) T cells.

Thus far our results demonstrate that LIT significantly enriches T cell activation in the TME. However, GC alone does not directly activate T cells (data not shown). To uncover how changes within the innate immune system allow for global $\mathrm{T}$ cell activation, we further analyzed modifications of the myeloid cells following LIT.

\section{LIT induces a proinflammatory phenotype in myeloid cells}

Within the immunosuppressive TME of an established tumor, TAMs generally resemble M2-like M $\varphi$ and inhibit migration and function of T cells $(33,34)$. TAMs play an essential role in the progression and metastasis of breast cancer $(35,36)$. With the dynamic role of TAMs, it is not surprising that TAMs residing in the TME have diverse functions $(21,37)$. To explore the heterogeneity of the myeloid cells, we used a higher SNN resolution (0.7) to re-cluster these immune cells. Using principal component analysis (PCA), we obtained 19 clusters (clusters 0-18), annotated using a series of commonly used immune cell genes (Figure 5A and S5A). We filtered out 4 non-myeloid clusters and kept 15 myeloid clusters for further analysis (Figure 5A). Comparing UMAPs of different treatment groups, we observed notable differences in the composition of granulocytic myeloid derived suppressor cells (G-MDSCs), M $\varphi$, and DCs in the LITtreated tumors (Figure S5B, red arrows). The proportions of immune cells in the 15 clusters were calculated for each group (Figure 5B). LIT enhanced the proportions of G- 
MDSC (clusters 3 and 8) and M1-like M $\varphi$ (cluster 7), but significantly decreased M2-like $\mathrm{M} \varphi$ (clusters 0 and 1), as shown in Figure 5B. Phenotypically, M1-like M $\varphi$ and M2-like $\mathrm{M} \varphi$ may be distinguished by cell surface expression of Itgam and Adgre1 (Figure S5C).

In addition to the myeloid cell proportions, we also investigated the alterations in their activation states. For the $M \varphi$ clusters, we calculated the DEGs and performed GSEA. First, we compared $M \varphi$ in LIT-treated and CTRL tumors. LIT increased proinflammatory responses of $\mathrm{M} \varphi$ in clusters 0,6 , and 2, compared to that in untreated tumors (Figure 5C-E). Specifically, these pathways include IFNy, TNF $\alpha$, and inflammatory responses of $\mathrm{M} 2$-like $\mathrm{M} \varphi$ in cluster 0 (Figure $5 \mathrm{C}$ ), and IFNy, TNF $\alpha$, and allograft rejection pathways of $M 2$-like $M \varphi$ in cluster 6 (Figure 5D). In cluster 2, M $\varphi$ precursors, IFN $\alpha$, IFNy, TNF $\alpha$, and UV response pathways were upregulated by LIT (Figure 5E). Next, we combined all M2-like M $\varphi$ clusters for GSEA and discovered LIT significantly upregulated IFN $\alpha$ and IFNy signaling pathways, and decreased hypoxia, TNF $\alpha$, and UV response pathways (Figure S5D). These results demonstrate that even though LIT-treated tumors contain $\mathrm{M} 2$-like $\mathrm{M} \varphi$, they are responding to type I IFNs and exhibiting a more proinflammatory phenotype than $\mathrm{M} 2$-like $\mathrm{M} \varphi$ from CTRL tumors.

Next, we analyzed G-MDSC gene expression in LIT treated tumors using gene ontology (GO) analysis. DEGs comparison between LIT and other groups was not practical due to low cell numbers in CTRL group (Figure 5B). GO analysis of G-MDSCs in LIT-treated tumors revealed 6 highly enriched pathways involved in neutrophil degranulation and interferon signaling (Figure S5E). These cytokine pathways are associated with proinflammatory responses and can enhance immunity. Additionally, signaling pathways of IL-10 and IL-13, the cytokines traditionally associated with MDSC immunosuppression, were also enriched in the G-MDSCs (Figure S5E). Interestingly, when we compared G-MDSCs in cluster 3 to cluster 8 in LIT-treated tumors, LIT enriched IFN $\alpha$ response genes in cluster 3, but reduced the TNF $\alpha$, UV response, apoptosis, hypoxia, epithelial to mesenchymal transition, p53, mTORC1, and MYC target pathways (Figure S5F). This is of particular interest because mTOR, hypoxia, and glycolysis signaling pathways are interconnected and they are key to MDSC suppressive function $(38,39)$. The reduction in these signaling pathways suggests a diminished immunosuppressive function of G-MDSCs in cluster 3 compared to that in 
cluster 8. Despite the increase in G-MDSCs, other key cell types ( $T$ cells, DCs, and TAMs) are activated and functional in the TME. Our findings suggest that the immunesuppressive function of G-MDSCs within the LIT-treated tumors is reduced.

Finally, we evaluated genes that did not comply to specific pathway networks with Venn diagrams of common DEGs across clusters 0, 2, 12, 3, and 4 in different groups: LIT vs CTRL, LIT vs PTT, and LIT vs GC. We found 13 conserved DEGs in LIT vs CTRL, 7 in LIT vs PTT, and 1 in LIT vs GC (Figure 5F, Table S2). Violin plots of these genes demonstrated that LIT significantly increased the expression of Ifi27I2a and Irf7 (Figure 5G). LIT also increased the expression of il1b, Btg2, Fos, Junb, Zfp36, Trim30a, Oasl2, Slfn5, and Sp100, and inhibited expression of Ninj1 (Figure S6A). Particularly interesting is the high expression of Irf7 in monocytes (cluster 12) from GCand LIT-treated tumors (Figure 5G), since Irf7 drives monocyte differentiation into M $\varphi$ (40). Cells in cluster 2 , the $M \varphi$ precursors, showed significantly upregulated Stat1 and displayed trending increases in the proportion of cells expressing Irf1, Irf8, and Irf5, genes expressed in activated M1-like M $\varphi$ (Figure 5G), likely explaining why LIT-treated

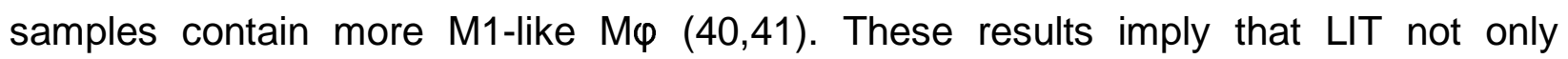
changes the behavior of existing $M 2$-like $M \varphi$, but also recruits and differentiates monocytes to become proinflammatory cells, enhancing antitumor immunity.

\section{LIT shifts the TME towards a proinflammatory phenotype, promoting monocyte differentiation into M1-like macrophages}

To determine the effect of LIT on the TME in driving monocytes towards M1-like M $\varphi$ and/or shifting M2-like $\mathrm{M} \varphi$ to $\mathrm{M} 1$-like, we conducted cell $\mathrm{CTI}$ analysis and discovered 7 major states. M2-like M $\varphi$ clusters were shown to reside within states 1, 2 and 7 (Figures 6A and S6B). M2-like $M \varphi$ in clusters 0, 1, and $M \varphi$ precursors in cluster 2 (Figure 6A), show a broader trajectory covering states 1, 2, 4, 6, and 7 (Figure S6B-C), indicating that these $\mathrm{M} \varphi$ were in a more plastic state and could be driven towards an $\mathrm{M} 1$ - or $\mathrm{M} 2$ like phenotype depending on the environmental signals they receive. Since the trajectories look identical between all four treatment groups, we conclude that cluster 0 and 1 are not the predominant source of $\mathrm{M} 1$-like $\mathrm{M} \varphi$ (Figure S6C). However, these M $\varphi$ 
clusters could be targets for M2 to M1 conversion therapeutics in combination with LIT or other immunotherapies.

The DCs (clusters 4, 14, and 16), G-MDSCs (clusters 3 and 8), and M1-like M $\varphi$ (cluster 7) were shown to reside in states 3, 4, 5, and 6 (Figure 6A and S6B). We

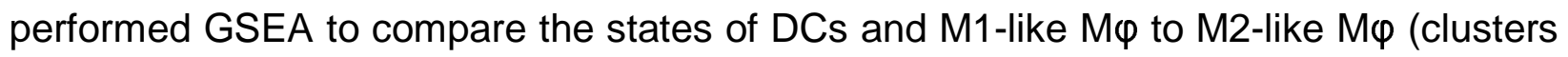

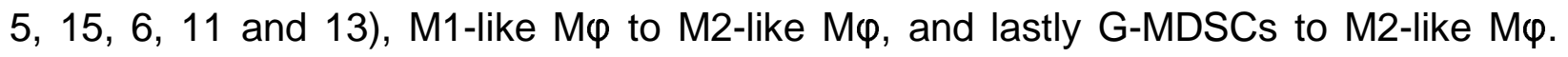
Although clusters 0 and 1 were also annotated as $M 2$-like $M \varphi$, they were not chosen due to their ubiquitous distributions along the trajectories. This comparison revealed significant enrichment of IFN $\alpha$, IFNy, TNF $\alpha$, and inflammatory responses in DCs and

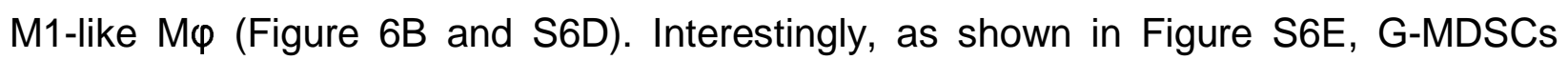
(clusters 3 and 8) displayed a significant upregulation of IFN $\alpha$, IFNy, and TNF $\alpha$ inflammatory responses, as well as immunosuppressive responses (apoptosis, hypoxia, and IL6/Jak/Stat3 signaling) compared to M2-like M $\varphi$. To make LIT treatment more successful in eliminating breast tumors, it is of future interest to combine this treatment with a G-MDSC inhibitor and/or anti-PD-L1 checkpoint therapy.

Based on the GSEA, we designated trajectory branches at states 1, 2, and 7 as anti-inflammatory, and states 4, 5, and 6 as proinflammatory (Figure 6C). Interestingly, when we separated the trajectory in Figure 6C into 4 treatment groups, we noticed that GC and LIT-treated tumors had a significantly larger proportion of cells residing in the proinflammatory state than in the anti-inflammatory state (Figure 6D-E). This demonstrates that LIT drives myeloid cells towards a proinflammatory phenotype.

To determine if the overall proinflammatory TME in GC and LIT-treated tumors contributes to the differentiation of the recruited monocytes towards $\mathrm{M} 1$-like $\mathrm{M} \varphi$ and/or monocyte-derived DCs (moDCs), we compared the cell trajectories of monocytes, M1like $\mathrm{M} \varphi$, and moDCs among the four treatment groups. Although LIT did not change the monocyte cell proportion (cluster 12 in Figure 5B), the monocyte cellular distribution was changed along the trajectory. The cells in the upper-left anti-inflammatory sides (states 1, 7, and 2) shifted towards the downward/lower-right proinflammatory sides (states 4, 5 and 6), in which the M1-like macrophages and moDCs reside (Figures 6F and S6B) suggesting that monocytes are the predominant source of $M 1$-like $M \varphi$. 
By using both Seurat and monocle2 analysis procedures, we conclude that LIT actively remodels the TME by shifting the cell composition and gene signatures from anti-inflammatory to proinflammatory. Additionally, we believe that the predominant source of the $M 1$-like $M \varphi$ is monocytes, not a result of $M 2$ to $M 1 M \varphi$ conversion. Lastly, the proinflammatory response generated in the myeloid compartment allows for the activation of T cells in the TME to potentiate antitumor immunity. 


\section{DISCUSSION}

In this study, scRNA-seq provided unbiased interrogation of immune cells residing within the TME of the MMTV-PyMT tumor model, to elucidate the immunological changes initiated by LIT from a cellular and transcriptional vantage point. We analyzed 49,380 TILs and characterized the cell types, cell states, and dynamic cellular transitions. LIT significantly changed the landscape of multiple immune cell types of both myeloid and lymphoid cells by increasing or decreasing several key immune cell populations, upregulating immune activation genes, and inducing a type I/II IFN cytokine signature in the TME. While GC also induced similar immune responses, GC alone, similar to PTT alone, could not slow or stop tumor growth. This indicates that increasing the inflammatory capacity of immune cells alone is not enough, and that it must be combined with disrupted tumor homeostasis to initiate antitumor functions.

Initial analysis of $\mathrm{T}$ cell activation and $\mathrm{T}$ cell depletion revealed that, in the MMTV-PyMT breast tumor model, $\mathrm{CD}^{+} \mathrm{T}$ cells are essential for the therapeutic effect of LIT (Figures 1F-G). This may not be the case for every tumor model, and our investigations on the necessity of $\mathrm{CD}^{+}{ }^{+} \mathrm{T}$ cells following LIT in other tumor models are ongoing. Further transcriptome analysis using scRNA-seq revealed that effector and memory T cells from LIT-treated tumors were responding to type I and II IFNs and transitioned to an activated state based on gene expression and CTI analysis (Figure 3E and Figures 4A-H). Interestingly, LIT dramatically increased the numbers of naïve $\mathrm{CD}^{+}$and $\mathrm{CD}^{+} \mathrm{T}$ cells in the TME, suggesting that LIT triggers active recruitment of naïve T cells (Figure 3B). That implies that LIT turns the TME into a tertiary lymphoid organ (TLO), which is a lymphoid-like structure where active antigen presentation and subsequent $B / T$ cell activation occur (42). This is of critical importance because TLOs are associated with higher TIL numbers, increased responses to ICT, and positive outcomes of cancer patients $(42,43)$. Supporting this notion, the naïve $T$ cells residing in the TME following LIT are responding to antigens as they have increased expression of genes involved in TCR signaling and activation, and have transitioned into an activated state (Figure 3E and Figure 4). Furthermore, TCR signaling and activation in naïve T 
cells portents that LIT promotes the generation of de novo antitumor T cell responses, not just enhances the existing anti-tumor $\mathrm{T}$ cell responses. This is important as immunologically "cold" tumors with low numbers of T cells could become "hot" following LIT, increasing the numbers of tumor specific T cells. Lastly, increasing the numbers and activity of tumor-infiltrating T cells makes LIT an encouraging treatment option to synergize with ICT, which relies on the number of T cells in the TME (44) and the activation state of these $\mathrm{T}$ cells (45). We speculate that the increased quality and improved quantity of T cells induced by LIT will synergize with ICT, allowing for reduced ICT doses, minimizing the potential for autoimmune side effects, and broadly increasing ICT efficacy across different cancers. Thus far, we have not noticed any autoimmune sequelae after LIT treatment, an observation that makes LIT with adjuvant ICT therapy particularly attractive.

A majority of the TAMs residing in the TME resemble M2-like M $\varphi$ and exhibit immunosuppressive functions (46). Our data revealed that in CTRL tumors the vast majority of myeloid cells are M2-like $\mathrm{M} \varphi$, with smaller populations of DCs and G-MDSCs (Figure 5B). LIT-treated tumors exhibited a dramatic increase in $M 1$-like $M \varphi$, and conversely a decrease in the two most prominent subtypes of M2-like M $\varphi$ (Figure 5B) that exhibit a broad trajectory by CTI analysis (Figure S6C). Although M2-like M $\varphi$ from clusters 0 and 1 hovered between both M1- and M2-like states, they do not appear to be the predominant source of $\mathrm{M} 1$-like $\mathrm{M} \varphi$ as all the treatment groups have cellular distribution that looks identical (Figure S6C). However, it is likely these $M \varphi$ retain plasticity and could be influenced by the local TME to become either M1- or M2-like M $\varphi$. Since clusters 0 and 1 are the predominant $M 2$-like $M \varphi$ populations within the TME, they make promising candidates for M2 to M1 conversion therapeutics (47), which could increase the clinical efficacy of LIT in breast cancer patients with high M2-like M $\varphi$ infiltration.

We next analyzed monocyte and $\mathrm{M} \varphi$ precursors in the TME to determine if these cells give rise to a majority of the $M 1$-like $M \varphi$. Gene expression and CTI analyses revealed that the monocytes recruited into the TME after LIT were poised to become $\mathrm{M} \varphi$ and moDCs (Figure 5B and 6F). Supporting this notion, monocytes from the LITtreated tumors have increased IRF7 expression (Figure 5G), which drives M $\varphi$ 
differentiation (40). In turn, the M $\varphi$ precursors (cluster 2) in LIT-treated tumors were poised to become $\mathrm{M} 1$-like $\mathrm{M} \varphi$ based on their increased expression of Stat1, Irf1, Irf5, Irf7, and Irf8 (Figure 5G), genes enriched in $\mathrm{M} 1 \mathrm{M} \varphi$ (41). CTI analysis also revealed that LIT significantly increased the numbers of myeloid cells residing in the proinflammatory state and decreased the numbers of cells residing in the anti-inflammatory state (Figure 6C-E).

We also observed a significant increase in granulocytic cells which were identified as G-MDSC (Figure 5B). Increased number of G-MDSC is another indicator that LIT drives a proinflammatory response, as MDSCs are normally recruited to high inflammation areas to promote immune suppression and wound healing. MDSCs are known immune suppressors and contribute to the establishment of the primary TME and a metastatic niche. Notably, G-MDSCs are less immunosuppressive than their monocytic-MDSC counterparts and are more similar to neutrophils (48). Gene ontology analysis revealed that the G-MDSCs from LIT-treated tumors responded to type I/II IFNs and also expressed the classic immunosuppressive signatures such as IL-10 and IL-13 signaling pathways (Figure S5E). Despite this immunosuppressive gene signature, LIT-treated tumors were enriched with activated $\mathrm{CD}^{+}$and $\mathrm{CD}^{+} \mathrm{T}$ cells (Figures 3 and 4), suggesting that the immunosuppressive function of the G-MDSCs may be reduced following LIT. However, we cannot discount the immunosuppressive ability of G-MDSCs as these cells express high levels of PD-L1, which may explain why a single application of LIT is not curative in this model (Figure 1B-C). Therefore, LIT combined with GMDSC inhibitors, or anti-PDL1 ICT, may greatly improve the efficacy of LIT in highly immunosuppressive tumors.

While the current study provided unequivocal support for the systemic antitumor effects of LIT, it also revealed some de novo mechanisms to be explored in order to further improve the efficacy of LIT. For example, ICT is a promising candidate to be synergized with T cells activated by LIT. Depending on the tumor type, M2-like M $\varphi$ and MDSC inhibitors make promising combinations to enhance the curative potential of LIT in highly immunosuppressive TMEs such as in triple negative breast cancer. By directly disrupting primary tumor homeostasis, we reversed the pro-tumor TME. Its ability to be 
bioRxiv preprint doi: https://doi.org/10.1101/2020.10.02.323006; this version posted October 3, 2020. The copyright holder for this preprint (which was not certified by peer review) is the author/funder. All rights reserved. No reuse allowed without permission.

customized with other appropriate therapies, dependent on the tumor immune profile, could make LIT an effective personalized immunotherapy for metastatic cancer patients. 


\section{ACKNOWLEDGMENTS}

This work was supported in part by the National Cancer Institute (R01CA205348 to WRC and 1R01CA223245 to ALW) and the Oklahoma Center for the Advancement of Science and Technology (HR16-085). We would like to thank the Flow Cytometry Core Facility and Genomics Core Facility at the Oklahoma Medical Research Foundation for providing flow cytometry services and assisting in scRNA-seq analysis. The authors (WRC, ARH, KL) also appreciate the generous support of Dr. and Mrs. Lee and Sherry Beasley, Dr. and Mrs. J. Michael and Kathy Steffen, and Dr. and Mrs. Damon and Heather Johnston. We thank Samuel Kelting for computational assistance. We also thank Dr. Samuel Siu Kit Lam from Immunophotonics for scientific input.

\section{AUTHOR CONTRIBUTIONS}

WRC, WHH, ARH conceived the project

$\mathrm{ARH}, \mathrm{CID}, \mathrm{KL}, \mathrm{JRK}, \mathrm{CLW}$, DM performed the experiments

$\mathrm{ARH}, \mathrm{KL}, \mathrm{CID}$ analyzed the data

$\mathrm{ARH}, \mathrm{KL}, \mathrm{WRC}$ completed the original draft of the manuscript

WHH, CID, XHS, ALW, JRK reviewed and edited the manuscript

ALW provided tumor samples

\section{DECLARATION OF INTERESTS}

Wei R. Chen is co-founder and an unpaid member of the Board of Directors of Immunophotonics, Inc.

All other authors declare no competing interests.

\section{KEY RESOURCES TABLE}

\begin{tabular}{|l|l|l|}
\hline REAGENT or RESOURCE & SOURCE & IDENTIFIER \\
\hline Antibody & Biolegend & cat\# 103134 \\
\hline CD45 (clone 30-F11) & Biolegend & cat\# 103112 \\
\hline CD45 (clone 30-F11) & Tonbo Bioscience & cat\# 35-0081-U500 \\
\hline CD8 (clone 53-6.7) &
\end{tabular}




\begin{tabular}{|c|c|c|}
\hline CD44 (clone IM7) & Tonbo Bioscience & cat\# 65-0441-U100 \\
\hline CD4 (clone GK1.5) & Biolegend & cat\# 100456 \\
\hline CD28 (clone 37.51) & Biolegend & cat\# 102104 \\
\hline CD62L (clone MEL-14) & Tonbo Bioscience & cat\# 60-0621-U100 \\
\hline CD3 (clone 17A2) & Tonbo Bioscience & cat\# 80-0032-U100 \\
\hline CD69 (clone H1.2F3) & Biolegend & cat\# 104530 \\
\hline CCR7 (CD197) (clone 4B12) & Biolegend & cat\# 120124 \\
\hline CD103 (clone 2E7) & Biolegend & cat\# 121414 \\
\hline Foxp3 (clone FJK-16s) & Invitrogen & cat\# 12-5773-82 \\
\hline CD25 (clone PC61) & Biolegend & cat\# 102012 \\
\hline CD103 (clone 2E7) & Biolegend & cat\# 121426 \\
\hline $\begin{array}{l}\text { CD326 (EpCAM) (clone } \\
\text { G8.8) }\end{array}$ & $\begin{array}{l}\text { Thermo Fisher } \\
\text { Scientific }\end{array}$ & cat\# 13-5791-82 \\
\hline $\begin{array}{l}\text { InVivoMAb anti-mouse } \\
\text { CD8a }\end{array}$ & BioXcell & cat\# BE0061 \\
\hline InVivoMAb anti-mouse CD4 & BioXcell & cat\# BP0003-1 \\
\hline $\begin{array}{l}\text { InVivoMAb rat lgG2b } \\
\text { isotype control, anti-keyhole } \\
\text { limpet hemocyanin }\end{array}$ & BioXcell & cat\# BE0090 \\
\hline \multicolumn{3}{|l|}{ Chemicals } \\
\hline Ghost dye & Tonbo Bioscience & cat\# 13-0870-T500 \\
\hline Strept-Avidin BV785 & Biolegend & cat\# 405249 \\
\hline $\begin{array}{l}\text { Collagenase, Type IV, } \\
\text { powder }\end{array}$ & $\begin{array}{l}\text { Thermo Fisher } \\
\text { Scientific }\end{array}$ & cat\# 17104019 \\
\hline DNase I recombinant & Sigma & cat\# 4536282001 \\
\hline $\begin{array}{l}\text { Corning RPMI } 1640 \text { Medium } \\
\text { (Mod.) }\end{array}$ & Fisher Scientific & cat\# MT50020PB \\
\hline $\begin{array}{l}\text { Corning }{ }^{\mathrm{TM}} \text { Lymphocyte } \\
\text { Separation Medium }\end{array}$ & VWR & cat\# 45000-726 \\
\hline \multicolumn{3}{|l|}{ Commercial Assay Kits } \\
\hline $\begin{array}{l}\text { Foxp3 / Transcription Factor } \\
\text { Staining Buffer Set }\end{array}$ & $\begin{array}{l}\text { Thermo Fisher } \\
\text { Scientific }\end{array}$ & Cat\# 00-5523-00 \\
\hline $\begin{array}{l}\text { EasySep }^{\mathrm{TM}} \text { Mouse } \\
\text { Streptavidin RapidSpheres }{ }^{\mathrm{TM}}\end{array}$ & Stem Cell & Cat\# 19860 \\
\hline
\end{tabular}




\begin{tabular}{|l|l|l|}
$\mid \begin{array}{l}\text { Isolation Kit } \\
\text { Experimental Models: Organisms/Strains }\end{array}$ \\
\hline FVB/NJ & Jackson Laboratory & Stock No: 001800 \\
\hline $\begin{array}{l}\text { FVB/N-Tg(MMTV- } \\
\text { PyVT)634Mul/J }\end{array}$ & Jackson Laboratory & Stock No: 002374 \\
\hline Software and Algorithms & FlowJo & $\begin{array}{l}\text { https://www.flowjo.com/solutions/flowjo/download } \\
\text { s/previous-versions }\end{array}$ \\
\hline FlowJo 10.6.1 & GraphPad Software & $\begin{array}{l}\text { https://www.graphpad.com/scientific- } \\
\text { software/prism/ }\end{array}$ \\
\hline GraphPad PRISM 7.0 & Butler et al., 2018 & https://satijalab.org/seurat/ \\
\hline Seurat 3.0 & Trapnell et al., 2014 & http://monocle-bio.sourceforge.net/ \\
\hline Monocle 2.0 &
\end{tabular}

\section{CONTACT FOR REAGENTS AND RESOURCE SHARING}

Further information and requests for resources and reagents should be directed to and will be fulfilled by the lead contact, Wei R. Chen (Wei-R-Chen@ou.edu).

\section{STUDY APPROVAL}

Experimental procedures and handling of mice were performed in accordance with the University of Oklahoma Health Sciences Center (OUHSC) and Oklahoma Medical Research Foundation (OMRF) Institutional Animal Care and Use Committee (IACUC) regulations and approved protocols.

\section{METHODS}

\section{MICE}

Female FVB/NJ wild-type (stock \#001800) and FVB/N transgenic MMTV-PyMT (stock \#002374) mice were purchased from Jackson Laboratories and housed according to institutional guidelines at the University of Oklahoma Health Sciences Center. All studies were approved and adhered to OUHSC Institutional Animal Care and Use Committee protocols (\#17-032-HCL). 


\section{SYNGENEIC TUMOR CELL TRANSPLANTATION}

PyMT murine breast tumor organoids were isolated from FVB/N-Tg (MMTVPyVT)634Mul/J mice as previously described (22). Briefly, cells were incubated overnight in mammary epithelial cell media (DMEM/F12 supplemented with $10 \%$ fetal bovine serum, $100 \mathrm{U} / \mathrm{mL}$ penicillin-streptomycin, $5 \mathrm{ug} / \mathrm{mL}$ insulin-transferrin-selenium, $1 \mathrm{ug} / \mathrm{mL}$ hydrocortisone, $10 \mathrm{ng} / \mathrm{mL}$ mouse epidermal growth factor (EGF), and $50 \mathrm{ug} / \mathrm{mL}$ gentamicin (Sigma-Aldrich, St. Louis, MO)). Cells were washed twice with Hepes Buffered Saline (HBSS), trypsinized, and resuspended to a concentration of $1 \times 10^{5}$ cell per 20uL. Cells were injected into the mammary fat pad of wild type FVB mice without clearing. The incision was closed with Vetbond tissue adhesive (3M).

\section{TREATMENT OF MOUSE TUMORS}

When tumors reached $0.5 \mathrm{~cm}^{3}$, the tumor site was shaved and mice received one of four treatments: CTRL (without treatment), GC alone, PTT alone, and LIT (PTT+GC). The mice in GC group received an intratumoral injection of $1 \%$ GC in $0.1 \mathrm{ml}$ solution (Immunophotonics, Inc.) For mice in PTT group, the tumor was treated by an 805-nm laser (AngioDynamics, Latham, NY) with a power density of $1 \mathrm{~W} / \mathrm{cm}^{2}$ for 10 minutes, using an optical fiber with a diffusion lens (Pioneer Optics, Bloomfield, CT) to delivery uniform light distribution on the treatment surface. For the mice in LIT group, the tumor was treated by the laser $\left(1 \mathrm{~W} / \mathrm{cm}^{2}\right.$ for 10 minutes), followed by an intratumoral injection of $\mathrm{GC}(0.1 \mathrm{ml}$ at $1 \%)$. Ten days after treatment, tumors were collected from selected mice in each group. The remaining mice were observed for up to 60 days or when the tumors reached a size of $2.5 \mathrm{~cm}^{3}$, or reached ethical endpoints.

\section{IN VIVO DEPLETION OF T CELLS}

Once tumors reached $0.4 \mathrm{~cm}^{3}, 400 \mu \mathrm{g}$ of anti-CD4, -CD8, or isotype antibody (BioXcell) was injected i.p. Twenty-four hours after antibody injection, tumors were treated with LIT or left untreated. Three days after LIT treatment, mice were injected with $200 \mu \mathrm{g}$ antibody, i.p. and maintained on this dose biweekly until tumors reached a size of $2.5 \mathrm{~cm}^{3}$ or reached ethical endpoints.

\section{TUMOR HARVEST}

Tumor tissues were isolated, minced, and digested with Collagenase IV and DNase I at $37^{\circ} \mathrm{C}$ for 20-30 minutes in RPMI. After enzymatic digestion, immune cells were enriched 
using lymphocyte separation medium (Corning). The enriched cells were then used for flow cytometry analysis.

\section{FACS AND CONVENTIONAL FLOW CYTOMETRY}

All samples were run on the LSR II and data was analyzed using FlowJo v10.6.1 (BD Biosciences, San Jose, CA). The cells used for scRNA-seq were sorted using the MoFlo sorting system. The antibodies used for flow cytometry are listed in the key resources.

\section{SINGLE-CELL RNA SEQUENCING}

\section{Sample Preparation}

In each of the four treatment groups, immune cells from four mice were pooled together for single cell sequencing. Tumor tissues were isolated, minced with scalpels, and they digested with Collagenase IV and Dnase I at $37^{\circ} \mathrm{C}$ for $20-30$ minutes. After enzymatic digestion, immune cells were enriched using lymphocyte separation medium. The enriched cells were then subjected to magnetic bead separation to remove the EpCAM ${ }^{+}$ cells. The EpCAM-depleted cells were stained with antibodies against CD45 and a viability dye. Live CD45+ cells were sorted using MoFlo and then processed for SCRNAseq via a 10x Genomics platform according to the manufacturer's instructions (10x Genomics). Paired-end RNA-seq was performed via an Illumina NovaSeq 6000 sequencing system. Sequencing reads were processed using the 10x Genomics CellRanger pipeline and further analyzed with the Seurat R package. The effect of mitochondrial gene representation and the variance of unique molecular identifier (UMI) counts were regressed out from the data set prior to analysis. Gene expression signatures defining cell clusters were analyzed after aggregating samples from all 4 treatment groups (CTRL, PTT, GC, LIT). The raw data from scRNA-seq experiments in this manuscript can be found in the NCBI's Gene Expression Omnibus database (GSE150675).

\section{ScRNA-seq Library Generation}

Droplet-based 3' end scRNA-seq was performed by encapsulating sorted live CD45 tumor-infiltrating immune cells into droplets using the 10x Genomics platform. cDNA libraries were prepared using Chromium Single Cell 3' Reagent Kits v3 according to the 
manufacturer's protocol (10x Genomics). The generated scRNA-seq libraries were sequenced using an Illumina NovaSeq 6000.

\section{Alignment, Barcode Assignment, UMI Counting}

The Cell Ranger (https://support.10xgenomics.com/single-cell-geneexpression/software/pipelines/latest/what-is-cell-ranger) was used to conduct sample demultiplexing, barcode processing, and single-cell 3' counting. The Linux command cellranger mkfastq was applied to demultiplex raw base call (BCL) files from the illumine NovaSeq6000 sequencer, into sample-specific fastq files. Then, fastq files for each sample were processed with cellranger count, which was used to align samples to mm10 genome, filter and quantify.

\section{Data Preprocessing with Seurat R Package}

Seurat-guided analyses (https://satijalab.org/seurat/vignettes.html) were used to preprocess and integrate datasets from different treatment groups (Butler et al., 2018). Genes that were expressed in less than 5 cells or cells that expressed less than 8000 and more than 6000 genes were excluded. Also, cells that expressed less than 512 and more than 92600 counts or with a mitochondria percentage over $10 \%$ were excluded. Most variable genes were identified using the FindVariableFeatures function by setting feature numbers as 2000. Principal component analysis (PCA) was performed on the scaled matrix (with most variable genes only) using the first 30 principal components (PCs). Both tSNE and UMAP dimensional reductions were carried out using the first 20 PCs to obtain two-dimensional representations of the cell states. For clustering, we used the function FindClusters that implements a shared nearest neighbor (SNN) modularity optimization-based clustering algorithm on $30 \mathrm{PCs}$ with resolution 0.5 for default analysis. For individual myeloid and lymphoid cell cluster analysis, 40 PCs and resolution 0.7 were used to gain more granularity and separate more clusters for immune cell subtypes.

\section{Identification of Cluster-specific Genes and Marker-based Classification}

Cells clusters were obtained by using the FindClusters function of the Seurat R package, which identifies clusters through an SNN modularity optimization-based algorithm. The biological cell type identities of each cluster were annotated with the assistance of an immune-cell scoring algorithm comparing the differentially expressed gene (DEG) 
signatures obtained from Seurat with the Immunological Genome Project Database (ImmGen) (24). This in silico cell type annotation was verified by using known canonical immune cell genes.

\section{Individual Myeloid and Lymphoid Population Analysis}

To increase the granularity, we manually used 40 PCs and 0.7 of SNN to separate myeloid and lymphoid clusters. This higher resolution approach allowed us to differentiate subsets of granulocytes, macrophages, dendritic cells, and T cell clusters.

\section{SINGLE CELL TRAJECTORY ANALYSIS}

Monocle2 was used to construct the single cell trajectory landscape of tumor immune infiltrates including myeloid cells, $\mathrm{CD}^{+} \mathrm{T}$ and $\mathrm{CD}^{+} \mathrm{T}$ cells (32). Seurat object was imported into Monocle2. Although monocle2 can provide cell type identification, we used Seurat to obtain cell type annotation information and generate trajectory using the differentialGeneTest function with the argument fullModelFormulaStr setting Seurat derived clusters. The data dimension reduction for trajectory was performed using the DDRTree algorithm in the Monocle package. Further information about Monocle can be found in Monocle's manual and vignette on Bioconductor.

\section{GSEA}

Differential Gene Expression was obtained by using the FindMarkers function in Seurat. MAST was used as the methodology. Ranked gene lists were then analyzed for gene set enrichment by using the clusterProfiler R package (49). Hallmark gene sets $(\mathrm{H})$ from the Molecular Signature Database (MSigDB, https://www.gsea-msigdb.org/gsea/msigdb) were used in these analyses (50). Curated gene sets (C2) and immunologic gene sets (C7) were also examined but not shown.

\section{STATISTICAL ANALYSES}

Evaluations for tumor growth and FACS data were conducted using one-way Anova. MAST test was used for analyzing differential gene expression in selected cell clusters. $P$ values less than or equal to 0.05 were considered statistically significant throughout $\left(^{*}\right.$, $\left.p \leq 0.05 ;{ }^{* *}, p \leq 0.01\right)$. 


\section{DATA AND SOFTWARE AVAILABILITY}

The accession number for the scRNAseq data reported in this paper is GEO:

GSE150675 (https://www.ncbi.nlm.nih.gov/geo/query/acc.cgi?acc=GSE150675).

Analysis of such data can also be available upon request. 


\section{REFERENCES}

1. Finn OJ. Cancer immunology. N Engl J Med 2008;358(25):2704-15 doi 10.1056/NEJMra072739.

2. Lambert AW, Pattabiraman DR, Weinberg RA. Emerging Biological Principles of Metastasis. Cell 2017;168(4):670-91 doi https://doi.org/10.1016/j.cell.2016.11.037.

3. Dougan M, Dranoff G, Dougan SK. Cancer Immunotherapy: Beyond Checkpoint Blockade. Annual Review of Cancer Biology 2019;3(1):55-75 doi 10.1146/annurev-cancerbio-030518-055552.

4. Berraondo P, Sanmamed MF, Ochoa MC, Etxeberria I, Aznar MA, Pérez-Gracia $\mathrm{JL}$, et al. Cytokines in clinical cancer immunotherapy. British journal of cancer 2019;120(1):6-15 doi 10.1038/s41416-018-0328-y.

5. Cruz E, Kayser V. Monoclonal antibody therapy of solid tumors: clinical limitations and novel strategies to enhance treatment efficacy. Biologics : targets \& therapy 2019;13:33-51 doi 10.2147/btt.s166310.

6. Cohen JE, Merims S, Frank S, Engelstein R, Peretz T, Lotem M. Adoptive cell therapy: past, present and future. Immunotherapy 2017;9(2):183-96 doi 10.2217/imt-2016-0112.

7. Rafiq S, Hackett CS, Brentjens RJ. Engineering strategies to overcome the current roadblocks in CAR T cell therapy. Nature reviews Clinical oncology 2020;17(3):147-67 doi 10.1038/s41571-019-0297-y.

8. DeMaria PJ, Bilusic M. Cancer Vaccines. Hematology/oncology clinics of North America 2019;33(2):199-214 doi 10.1016/j.hoc.2018.12.001.

9. Couzin-Frankel J. Breakthrough of the year 2013. Cancer immunotherapy. Science 2013;342(6165):1432-3 doi 10.1126/science.342.6165.1432.

10. Sambi M, Bagheri L, Szewczuk MR. Current Challenges in Cancer Immunotherapy: Multimodal Approaches to Improve Efficacy and Patient Response Rates. Journal of oncology 2019;2019:4508794 doi $10.1155 / 2019 / 4508794$.

11. Hegde PS, Chen DS. Top 10 Challenges in Cancer Immunotherapy. Immunity 2020;52(1):17-35 doi 10.1016/j.immuni.2019.12.011.

12. Chen WR, Carubelli R, Liu H, Nordquist RE. Laser immunotherapy. Molecular Biotechnology 2003;25(1):37-43 doi 10.1385/MB:25:1:37.

13. Zhou F, Li X, Naylor MF, Hode T, Nordquist RE, Alleruzzo L, et al. InCVAX--a novel strategy for treatment of late-stage, metastatic cancers through photoimmunotherapy induced tumor-specific immunity. Cancer letters 2015;359(2):169-77 doi 10.1016/j.canlet.2015.01.029.

14. Chen WR, Jeong SW, Lucroy MD, Wolf RF, Howard EW, Liu H, et al. Induced antitumor immunity against DMBA-4 metastatic mammary tumors in rats using laser immunotherapy. International journal of cancer 2003;107(6):1053-7 doi 10.1002/ijc. 11501.

15. Chen WR, Zhou F, Yang J, Zhang Y, Liu M, Lang ML, et al. Local phototherapy synergizes with immunoadjuvant for treatment of pancreatic cancer through induced immunogenic tumor vaccine. Clinical Cancer Research 2018 doi 10.1158/1078-0432.ccr-18-1126. 
16. Li X, Ferrel GL, Guerra MC, Hode T, Lunn JA, Adalsteinsson O, et al. Preliminary safety and efficacy results of laser immunotherapy for the treatment of metastatic breast cancer patients. Photochemical \& Photobiological Sciences 2011;10(5):817-21 doi 10.1039/COPP00306A.

17. Li X, Naylor MF, Le H, Nordquist RE, Teague TK, Howard CA, et al. Clinical effects of in situ photoimmunotherapy on late-stage melanoma patients: a preliminary study. Cancer Biol Ther 2010;10(11):1081-7 doi 10.4161/cbt.10.11.13434.

18. Naylor MF, Chen WR, Teague TK, Perry LA, Nordquist RE. In situ photoimmunotherapy: a tumour-directed treatment for melanoma. The British journal of dermatology 2006;155(6):1287-92 doi 10.1111/j.13652133.2006.07514.x.

19. Guy CT, Cardiff RD, Muller WJ. Induction of mammary tumors by expression of polyomavirus middle $T$ oncogene: a transgenic mouse model for metastatic disease. Mol Cell Biol 1992;12(3):954-61 doi 10.1128/mcb.12.3.954.

20. Rennhack JP, To B, Swiatnicki M, Dulak C, Ogrodzinski MP, Zhang Y, et al. Integrated analyses of murine breast cancer models reveal critical parallels with human disease. Nature Communications 2019;10(1):3261 doi 10.1038/s41467019-11236-3.

21. Azizi E, Carr AJ, Plitas G, Cornish AE, Konopacki C, Prabhakaran S, et al. Single-Cell Map of Diverse Immune Phenotypes in the Breast Tumor Microenvironment. Cell 2018;174(5):1293-308.e36 doi 10.1016/j.cell.2018.05.060.

22. Smith BA, Shelton DN, Kieffer C, Milash B, Usary J, Perou CM, et al. Targeting the PyMT Oncogene to Diverse Mammary Cell Populations Enhances Tumor Heterogeneity and Generates Rare Breast Cancer Subtypes. Genes Cancer 2012;3(9-10):550-63 doi 10.1177/1947601913475359.

23. Butler A, Hoffman P, Smibert P, Papalexi E, Satija R. Integrating single-cell transcriptomic data across different conditions, technologies, and species. Nature biotechnology 2018;36(5):411-20 doi 10.1038/nbt.4096.

24. Ekiz HA, Huffaker TB, Grossmann AH, Stephens WZ, Williams MA, Round JL, et al. MicroRNA-155 coordinates the immunological landscape within murine melanoma and correlates with immunity in human cancers. JCl insight 2019;4(6) doi 10.1172/jci.insight.126543.

25. Desai DM, Sap J, Silvennoinen O, Schlessinger J, Weiss A. The catalytic activity of the CD45 membrane-proximal phosphatase domain is required for TCR signaling and regulation. The EMBO journal 1994;13(17):4002-10.

26. Filén S, Lahesmaa R. GIMAP Proteins in T-Lymphocytes. Journal of signal transduction 2010;2010:268589 doi 10.1155/2010/268589.

27. Yeung T-L, Tsai CC, Leung CS, Au Yeung C-L, Thompson MS, Lu KH, et al. ISG15 Promotes ERK1 ISGylation, CD8+ T Cell Activation and Suppresses Ovarian Cancer Progression. Cancers (Basel) 2018;10(12):464 doi 10.3390/cancers10120464.

28. Zhang Y, Reynolds JM, Chang SH, Martin-Orozco N, Chung Y, Nurieva RI, et al. MKP-1 is necessary for T cell activation and function. The Journal of biological chemistry 2009;284(45):30815-24 doi 10.1074/jbc.M109.052472. 
29. Rincón M, Flavell RA. AP-1 transcriptional activity requires both T-cell receptormediated and co-stimulatory signals in primary $\mathrm{T}$ lymphocytes. The EMBO journal 1994;13(18):4370-81.

30. Wabnitz GH, Köcher T, Lohneis P, Stober C, Konstandin MH, Funk B, et al. Costimulation induced phosphorylation of L-plastin facilitates surface transport of the T cell activation molecules CD69 and CD25. Eur J Immunol 2007;37(3):64962 doi 10.1002/eji.200636320.

31. Kruglova NA, Meshkova TD, Kopylov AT, Mazurov DV, Filatov AV. Constitutive and activation-dependent phosphorylation of lymphocyte phosphataseassociated phosphoprotein (LPAP). PLoS One 2017;12(8):e0182468 doi 10.1371/journal.pone.0182468.

32. Qiu X, Mao Q, Tang Y, Wang L, Chawla R, Pliner HA, et al. Reversed graph embedding resolves complex single-cell trajectories. Nature methods 2017;14(10):979-82 doi 10.1038/nmeth.4402.

33. Peranzoni E, Lemoine J, Vimeux L, Feuillet V, Barrin S, Kantari-Mimoun C, et al. Macrophages impede CD8 T cells from reaching tumor cells and limit the efficacy of anti-PD-1 treatment. Proceedings of the National Academy of Sciences of the United States of America 2018;115(17):E4041-E50 doi 10.1073/pnas.1720948115.

34. Li X, Liu R, Su X, Pan Y, Han X, Shao C, et al. Harnessing tumor-associated macrophages as aids for cancer immunotherapy. Molecular Cancer 2019;18(1):177 doi 10.1186/s12943-019-1102-3.

35. Kitamura T, Doughty-Shenton D, Cassetta L, Fragkogianni S, Brownlie D, Kato Y, et al. Monocytes Differentiate to Immune Suppressive Precursors of MetastasisAssociated Macrophages in Mouse Models of Metastatic Breast Cancer. Frontiers in immunology 2018;8:2004- doi 10.3389/fimmu.2017.02004.

36. Lin EY, Nguyen AV, Russell RG, Pollard JW. Colony-stimulating factor 1 promotes progression of mammary tumors to malignancy. The Journal of experimental medicine 2001;193(6):727-40 doi 10.1084/jem.193.6.727.

37. Qian B-Z, Pollard JW. Macrophage diversity enhances tumor progression and metastasis. Cell 2010;141(1):39-51 doi 10.1016/j.cell.2010.03.014.

38. Deng Y, Yang J, Luo F, Qian J, Liu R, Zhang D, et al. mTOR-mediated glycolysis contributes to the enhanced suppressive function of murine tumor-infiltrating monocytic myeloid-derived suppressor cells. Cancer Immunol Immunother 2018;67(9):1355-64 doi 10.1007/s00262-018-2177-1.

39. Li W, Tanikawa T, Kryczek I, Xia H, Li G, Wu K, et al. Aerobic Glycolysis Controls Myeloid-Derived Suppressor Cells and Tumor Immunity via a Specific CEBPB Isoform in Triple-Negative Breast Cancer. Cell Metab 2018;28(1):87-103.e6 doi 10.1016/j.cmet.2018.04.022.

40. Günthner R, Anders H-J. Interferon-regulatory factors determine macrophage phenotype polarization. Mediators Inflamm 2013;2013:731023- doi 10.1155/2013/731023.

41. Tugal D, Liao X, Jain MK. Transcriptional control of macrophage polarization. Arterioscler Thromb Vasc Biol 2013;33(6):1135-44 doi 10.1161/ATVBAHA.113.301453. 
42. de Chaisemartin L, Goc J, Damotte D, Validire $P$, Magdeleinat $P$, Alifano $M$, et al. Characterization of Chemokines and Adhesion Molecules Associated with T cell Presence in Tertiary Lymphoid Structures in Human Lung Cancer. Cancer Research 2011;71(20):6391-9 doi 10.1158/0008-5472.can-11-0952.

43. Lin L, Hu X, Zhang H, Hu H. Tertiary Lymphoid Organs in Cancer Immunology: Mechanisms and the New Strategy for Immunotherapy. Frontiers in Immunology 2019;10(1398) doi 10.3389/fimmu.2019.01398.

44. Ayers M, Lunceford J, Nebozhyn M, Murphy E, Loboda A, Kaufman DR, et al. IFN- $y$-related mRNA profile predicts clinical response to PD-1 blockade. J Clin Invest 2017;127(8):2930-40 doi 10.1172/jci91190.

45. Prat A, Navarro A, Paré L, Reguart N, Galván P, Pascual T, et al. ImmuneRelated Gene Expression Profiling After PD-1 Blockade in Non-Small Cell Lung Carcinoma, Head and Neck Squamous Cell Carcinoma, and Melanoma. Cancer Res 2017;77(13):3540-50 doi 10.1158/0008-5472.can-16-3556.

46. Zheng X, Turkowski K, Mora J, Brüne B, Seeger W, Weigert A, et al. Redirecting tumor-associated macrophages to become tumoricidal effectors as a novel strategy for cancer therapy. Oncotarget 2017;8(29):48436-52 doi 10.18632/oncotarget.17061.

47. Lin Y, Xu J, Lan H. Tumor-associated macrophages in tumor metastasis: biological roles and clinical therapeutic applications. Journal of Hematology \& Oncology 2019;12(1):76 doi 10.1186/s13045-019-0760-3.

48. Ugel S, De Sanctis F, Mandruzzato S, Bronte V. Tumor-induced myeloid deviation: when myeloid-derived suppressor cells meet tumor-associated macrophages. J Clin Invest 2015;125(9):3365-76 doi 10.1172/JCI80006.

49. Yu G, Wang LG, Han Y, He QY. clusterProfiler: an R package for comparing biological themes among gene clusters. Omics : a journal of integrative biology 2012;16(5):284-7 doi 10.1089/omi.2011.0118.

50. Liberzon A. A description of the Molecular Signatures Database (MSigDB) Web site. Methods in molecular biology (Clifton, NJ) 2014;1150:153-60 doi 10.1007/978-1-4939-0512-6_9. 


\section{Figure Legends}

Figure 1. LIT treatment slows tumor growth by activating tumor-infiltrating $\mathrm{CD}^{+} \mathrm{T}$ cells.

A: Schematic of MMTV-PyMT tumor implantation, treatment, and analysis.

B: Individual and mean tumor size of mice after different treatments.

C: Survival rates of tumor-bearing mice. Log-rank (Mantel-Cox) test was used for statistical analysis.

D: T-SNE plots generated from 3 individual concatenated files in Flowjo of tumorinfiltrating $\mathrm{CD}^{+} \mathrm{T}$ cells from different treatment groups. Manual gates for CD44 and CD62L were overlayed onto the T-SNE plots. The colors indicate the expression of different cell surface marker combinations. T cells were analyzed 10 days after treatment.

$\mathrm{E}$ : The MFI of CD69 and CD28 on CD8 ${ }^{+}$T cells, normalized to the CTRL, 10 days after treatment. One-way ANOVA was used for statistical analysis.

F. Individual tumor size of untreated or LIT-treated mice that were injected with isotype antibodies or $\mathrm{CD}^{+}$or $\mathrm{CD} 8^{+}$depletion antibodies.

G. Survival rates of untreated or LIT-treated tumor-bearing mice injected with isotype antibodies or depleted of $\mathrm{CD}^{+}$or $\mathrm{CD}^{+} \mathrm{T}$ cells. Log-rank (Mantel-Cox) test was used for statistical analysis.

Figure 2. ScRNA-seq of CTRL, GC, PTT, and LIT treated tumors creates a tumorinfiltrating immune cell atlas.

A: Two-dimensional visualization of single cell clusters using method of t-distributed Stochastic Neighbor Embedding (t-SNE) from integrated immune cells of different treatment groups. 
B: Two-dimensional visualization of single cell clusters using method of Uniform Manifold Approximation and Projection (UMAP) from integrated immune cells of different treatment groups.

C: Heatmap of gene expression for the top 10 DEGs in each cluster (yellow bars).

D: Validation of unsupervised clustering using traditional immune cell genes.

E: UMAP plots of the immune cell atlas from individual treatment groups.

F. Proportion of lymphoid cells in different clusters compared to total lymphocytes in different treatment groups. Statistical analysis was performed using proportion test function (prop.test) in R.

G: Proportion of myeloid cells in different clusters compared to total myeloid cells in individual treatment groups. Statistical analysis was performed using proportion test function (prop.test) in R.

Figure 3. LIT treatment enriches proinflammatory signaling pathways in TILs.

A: UMAP of re-clustered lymphoid cells with an increased SNN resolution (from 0.5 to 0.7). The insert is the clusters of lymphoid cells with the lower SNN (0.5) in Figure 2B.

B: Proportions of re-clustered lymphoid cells in different treatment groups.

C: Heatmap of pathway enrichment for lymphoid cell clusters induced by LIT using GSEA.

D: Venn diagrams showing the number of DEGs and commonly expressed genes in clusters $0,3,5 / 6,1$, and 9 .

E: Expression levels of 4 representative DEGs identified in the Venn diagrams from cluster $0,3,5 / 6$, and 9 .

Figure 4. T cells from LIT-treated tumors reside in activated states. 
A: Branched trajectory of $\mathrm{CD}^{+} \mathrm{T}$ cells in different clusters separated according to each annotated cell type.

B: Branched trajectory of $\mathrm{CD}^{+} \mathrm{T}$ cells separated according to treatment group.

C: LIT induced transitioning (purple arrow) pattern for $\mathrm{CD8}^{+} \mathrm{T}$ cells from a resting state (blue circle) to an activated state (red circle).

D: Branched trajectories of $\mathrm{CD}^{+} \mathrm{T}$ cell subtypes in different treatment groups. Cells in blue circles are in a resting state and cells in red circles are in an activated state.

E: Branched trajectory of $\mathrm{CD}^{+} \mathrm{T}$ cells in different clusters separated according to each annotated cell type.

F: Branched trajectory of $\mathrm{CD}^{+} \mathrm{T}$ cells separated according to treatment group.

G: LIT/GC induced transitioning (purple arrow) pattern for $\mathrm{CD}^{+} \mathrm{T}$ cells from a resting state (blue circle) to an activated state (red circle).

$\mathrm{H}$ : Branched trajectories of $\mathrm{CD}^{+} \mathrm{T}$ cell subtypes in different treatment groups. Cells blue circle are in a resting state and cells in red circles are in an activated state.

I: Pathway enrichment for all CD8 ${ }^{+}$T cell clusters induced by LIT using GSEA.

J: Pathway enrichment for all CD4 ${ }^{+}$T cell clusters induced by LIT using GSEA.

Figure 5. LIT primes tumor-resident innate immune cells to proinflammatory states.

A: UMAP of re-clustered myeloid cells with an increased SNN resolution (from 0.5 to 0.7). The insert is the clusters of myeloid cells with the lower SNN (0.5) in Figure 2B.

B: The proportions of re-clustered myeloid cells from individual treatment groups. Statistical analysis was performed using proportion test function (prop.test) in R.

C-E: Pathway enrichment for representative myeloid cells (clusters 0, 6, and 2) induced by LIT using GSEA. 
F: Venn diagrams showing the number of DEGs and commonly expressed genes identified in each myeloid cell cluster.

G: Expression levels of select genes upregulated in cluster 2 and 12.

Figure 6. LIT promotes tumor-infiltrating monocytes differentiation to become proinflammatory cell types.

A: Single cell trajectory inference of re-clustered myeloid cells in different treatment groups.

B: Pathway enrichment for DEGs by comparing proinflammatory trajectories to antiinflammatory using GSEA. Clusters 7, 14, and 16 (proinflammatory) were compared to clusters $5,6,11,13$, and 15 (anti-inflammatory).

C: LIT/GC induced transitioning (purple arrow) pattern for myeloid cells from an antiinflammatory state (blue circle) to a proinflammatory state (red circle).

D: Branched trajectories of selected myeloid cells in different treatment groups. Blue circles indicate anti-inflammatory (pro-tumor) trajectories while red circles indicate proinflammatory (anti-tumor) trajectories

E: Cell proportions from anti-inflammatory and proinflammatory myeloid trajectories in different treatment groups. Statistical analysis was performed using proportion test function (prop.test) in $\mathrm{R} .{ }^{* *}$ means $p<0.01$.

F: Branched trajectories of selected myeloid cells (M1 macrophages of cluster 7, Monocytes of cluster 12, and Monocyte-derived DCs of cluster 14) in different treatment groups. 


\section{Figure Legends}

Figure 1. LIT treatment slows tumor growth by activating tumor-infiltrating $\mathrm{CD}^{+} \mathrm{T}$ cells.

A: Schematic of MMTV-PyMT tumor implantation, treatment, and analysis.

B: Individual and mean tumor size of mice after different treatments.

C: Survival rates of tumor-bearing mice. Log-rank (Mantel-Cox) test was used for statistical analysis.

D: T-SNE plots generated from 3 individual concatenated files in Flowjo of tumorinfiltrating $\mathrm{CD}^{+} \mathrm{T}$ cells from different treatment groups. Manual gates for CD44 and CD62L were overlayed onto the T-SNE plots. The colors indicate the expression of different cell surface marker combinations. T cells were analyzed 10 days after treatment.

$\mathrm{E}$ : The MFI of CD69 and CD28 on CD8 ${ }^{+}$T cells, normalized to the CTRL, 10 days after treatment. One-way ANOVA was used for statistical analysis.

F. Individual tumor size of untreated or LIT-treated mice that were injected with isotype antibodies or $\mathrm{CD}^{+}$or $\mathrm{CD} 8^{+}$depletion antibodies.

G. Survival rates of untreated or LIT-treated tumor-bearing mice injected with isotype antibodies or depleted of $\mathrm{CD}^{+}$or $\mathrm{CD}^{+} \mathrm{T}$ cells. Log-rank (Mantel-Cox) test was used for statistical analysis.

Figure 2. ScRNA-seq of CTRL, GC, PTT, and LIT treated tumors creates a tumorinfiltrating immune cell atlas.

A: Two-dimensional visualization of single cell clusters using method of t-distributed Stochastic Neighbor Embedding ( $t-S N E$ ) from integrated immune cells of different treatment groups. 
B: Two-dimensional visualization of single cell clusters using method of Uniform Manifold Approximation and Projection (UMAP) from integrated immune cells of different treatment groups.

C: Heatmap of gene expression for the top 10 DEGs in each cluster (yellow bars).

D: Validation of unsupervised clustering using traditional immune cell genes.

E: UMAP plots of the immune cell atlas from individual treatment groups.

F. Proportion of lymphoid cells in different clusters compared to total lymphocytes in different treatment groups. Statistical analysis was performed using proportion test function (prop.test) in R.

G: Proportion of myeloid cells in different clusters compared to total myeloid cells in individual treatment groups. Statistical analysis was performed using proportion test function (prop.test) in R.

Figure 3. LIT treatment enriches proinflammatory signaling pathways in TILs.

A: UMAP of re-clustered lymphoid cells with an increased SNN resolution (from 0.5 to 0.7). The insert is the clusters of lymphoid cells with the lower SNN (0.5) in Figure 2B.

B: Proportions of re-clustered lymphoid cells in different treatment groups.

C: Heatmap of pathway enrichment for lymphoid cell clusters induced by LIT using GSEA.

D: Venn diagrams showing the number of DEGs and commonly expressed genes in clusters $0,3,5 / 6,1$, and 9 .

E: Expression levels of 4 representative DEGs identified in the Venn diagrams from cluster $0,3,5 / 6$, and 9 .

Figure 4. T cells from LIT-treated tumors reside in activated states.

A: Branched trajectory of $\mathrm{CD}^{+} \mathrm{T}$ cells in different clusters separated according to each annotated cell type.

B: Branched trajectory of $\mathrm{CD} 8^{+} \mathrm{T}$ cells separated according to treatment group. 
C: LIT induced transitioning (purple arrow) pattern for $\mathrm{CD}^{+} \mathrm{T}$ cells from a resting state (blue circle) to an activated state (red circle).

D: Branched trajectories of $\mathrm{CD}^{+} \mathrm{T}$ cell subtypes in different treatment groups. Cells in blue circles are in a resting state and cells in red circles are in an activated state.

$\mathrm{E}$ : Branched trajectory of $\mathrm{CD} 4^{+} \mathrm{T}$ cells in different clusters separated according to each annotated cell type.

F: Branched trajectory of $C D 4^{+} \mathrm{T}$ cells separated according to treatment group.

G: LIT/GC induced transitioning (purple arrow) pattern for $\mathrm{CD}^{+} \mathrm{T}$ cells from a resting state (blue circle) to an activated state (red circle).

$\mathrm{H}$ : Branched trajectories of $\mathrm{CD}^{+} \mathrm{T}$ cell subtypes in different treatment groups. Cells blue circle are in a resting state and cells in red circles are in an activated state.

I: Pathway enrichment for all CD8 ${ }^{+} \mathrm{T}$ cell clusters induced by LIT using GSEA.

J: Pathway enrichment for all CD4 ${ }^{+} \mathrm{T}$ cell clusters induced by LIT using GSEA.

Figure 5. LIT primes tumor-resident innate immune cells to proinflammatory states.

A: UMAP of re-clustered myeloid cells with an increased SNN resolution (from 0.5 to $0.7)$. The insert is the clusters of myeloid cells with the lower SNN (0.5) in Figure 2B.

B: The proportions of re-clustered myeloid cells from individual treatment groups. Statistical analysis was performed using proportion test function (prop.test) in R.

C-E: Pathway enrichment for representative myeloid cells (clusters 0,6 , and 2) induced by LIT using GSEA.

F: Venn diagrams showing the number of DEGs and commonly expressed genes identified in each myeloid cell cluster.

G: Expression levels of select genes upregulated in cluster 2 and 12.

Figure 6. LIT promotes tumor-infiltrating monocytes differentiation to become proinflammatory cell types. 
A: Single cell trajectory inference of re-clustered myeloid cells in different treatment groups.

B: Pathway enrichment for DEGs by comparing proinflammatory trajectories to antiinflammatory using GSEA. Clusters 7, 14, and 16 (proinflammatory) were compared to clusters $5,6,11,13$, and 15 (anti-inflammatory).

C: LIT/GC induced transitioning (purple arrow) pattern for myeloid cells from an antiinflammatory state (blue circle) to a proinflammatory state (red circle).

D: Branched trajectories of selected myeloid cells in different treatment groups. Blue circles indicate anti-inflammatory (pro-tumor) trajectories while red circles indicate proinflammatory (anti-tumor) trajectories

E: Cell proportions from anti-inflammatory and proinflammatory myeloid trajectories in different treatment groups. Statistical analysis was performed using proportion test function (prop.test) in R. ${ }^{* *}$ means $p<0.01$.

F: Branched trajectories of selected myeloid cells (M1 macrophages of cluster 7, Monocytes of cluster 12, and Monocyte-derived DCs of cluster 14) in different treatment groups. 
PyMT spontaneous tumor implantation into wild type FVB Mice

Tumor treatment at $0.5 \mathrm{~cm}^{3}$ at Day 0
Collection of tumor at Day 10
Day 60 or once tumor volume reaches $2.5 \mathrm{~cm}^{3}$
B
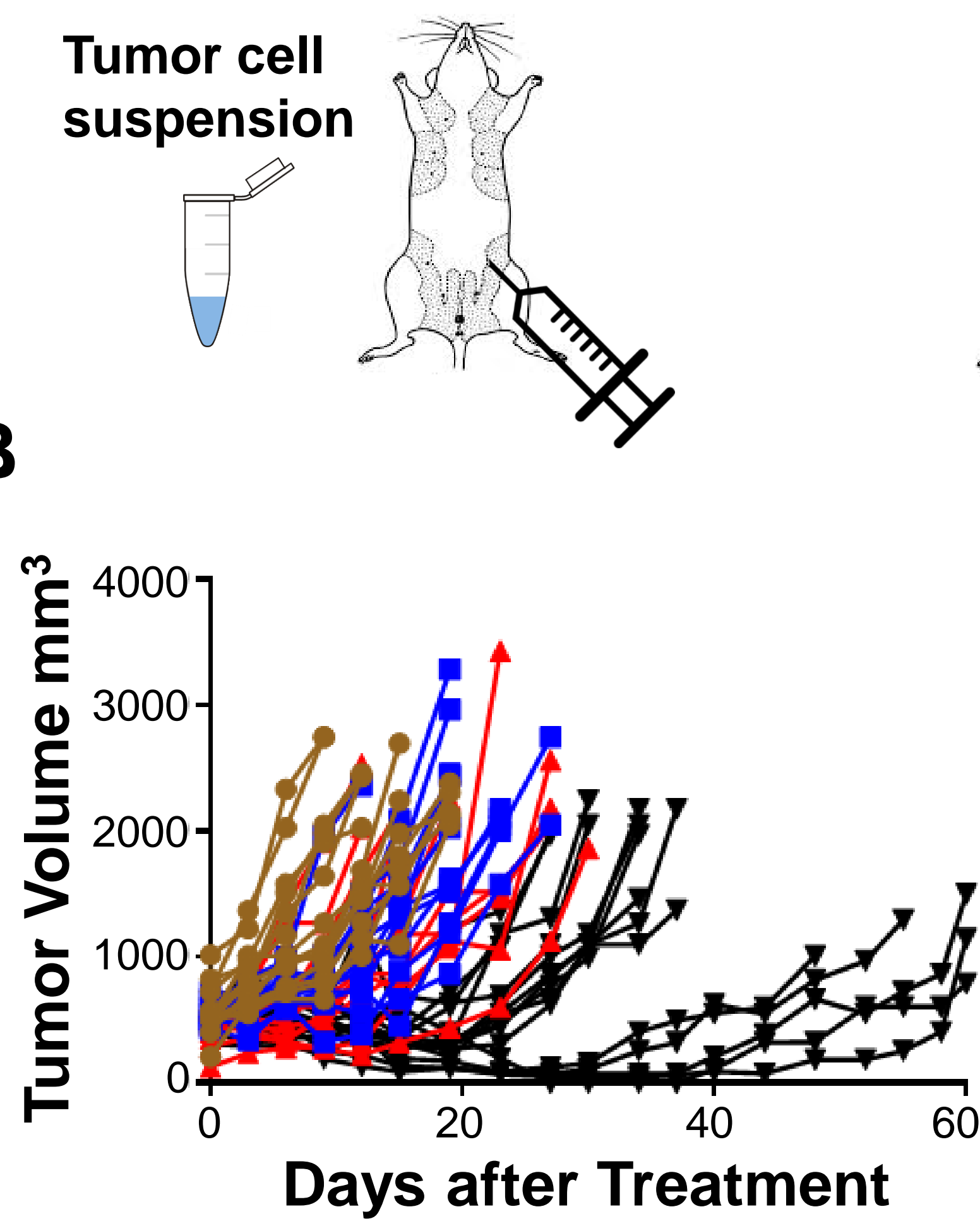

D

D

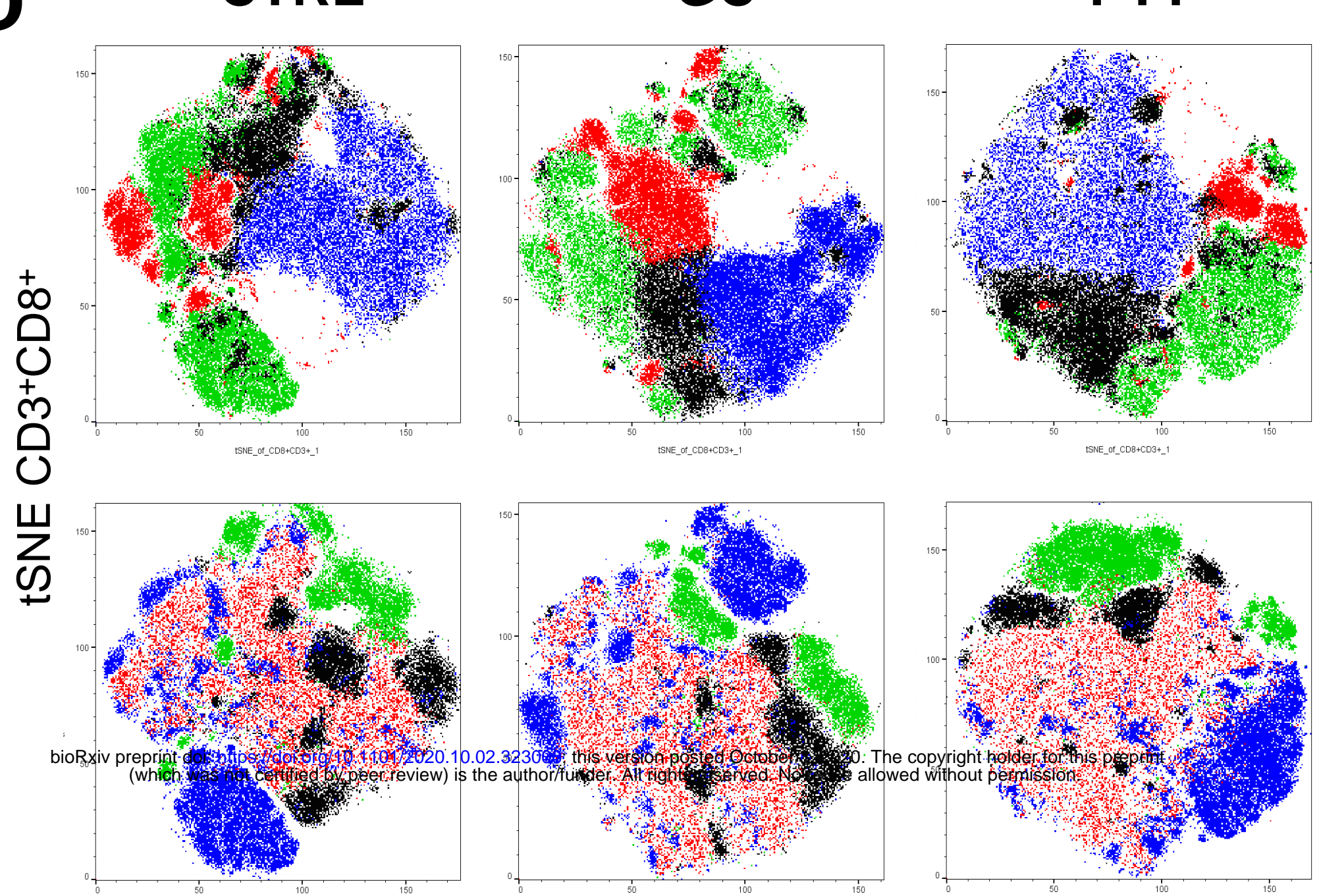

tSNE CD3+CD8+

E

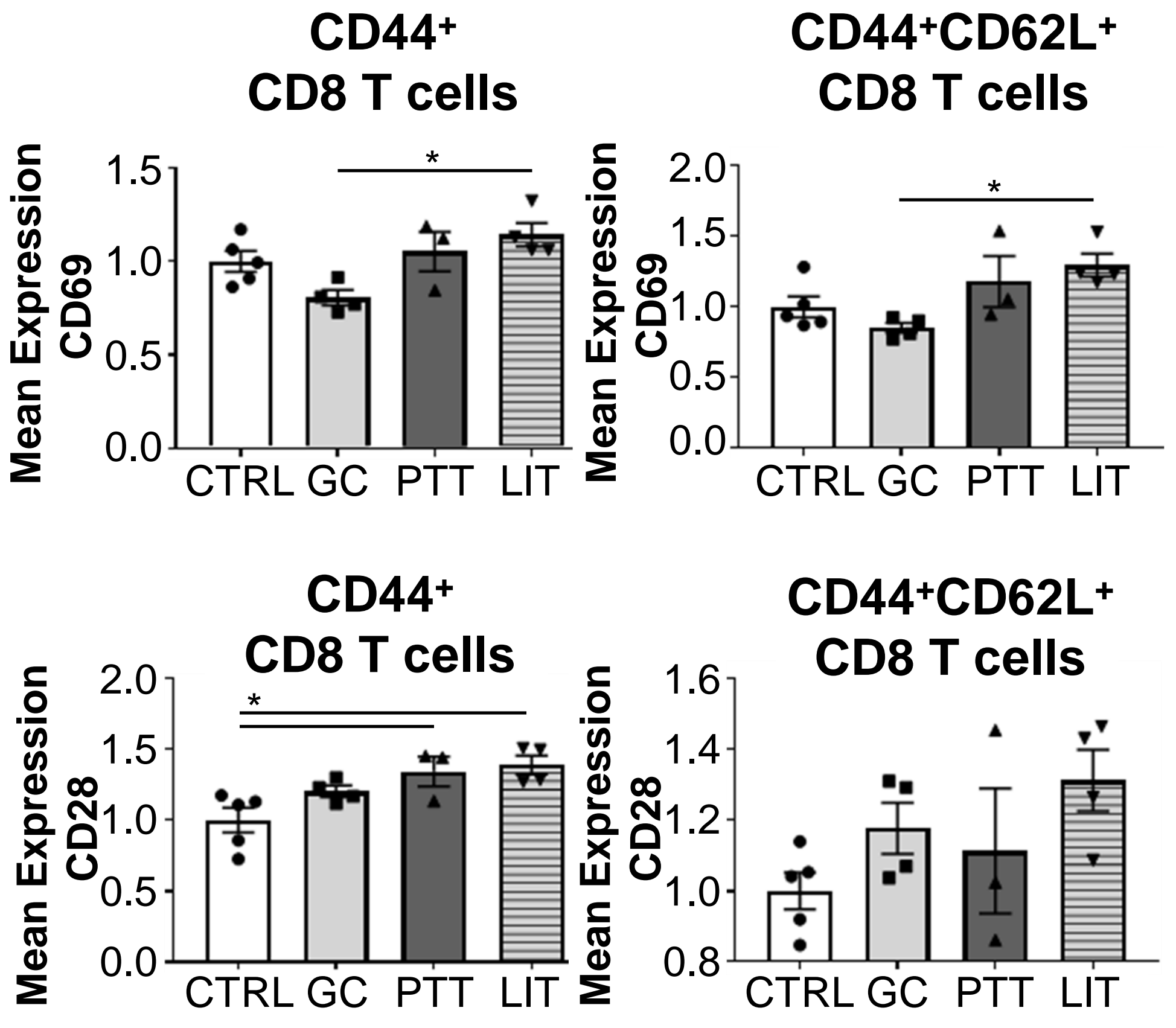

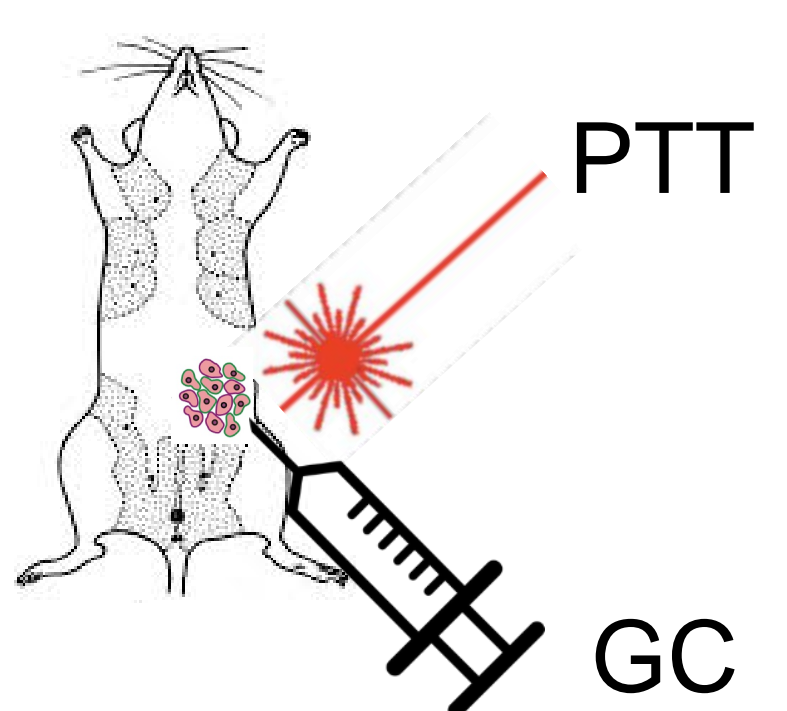
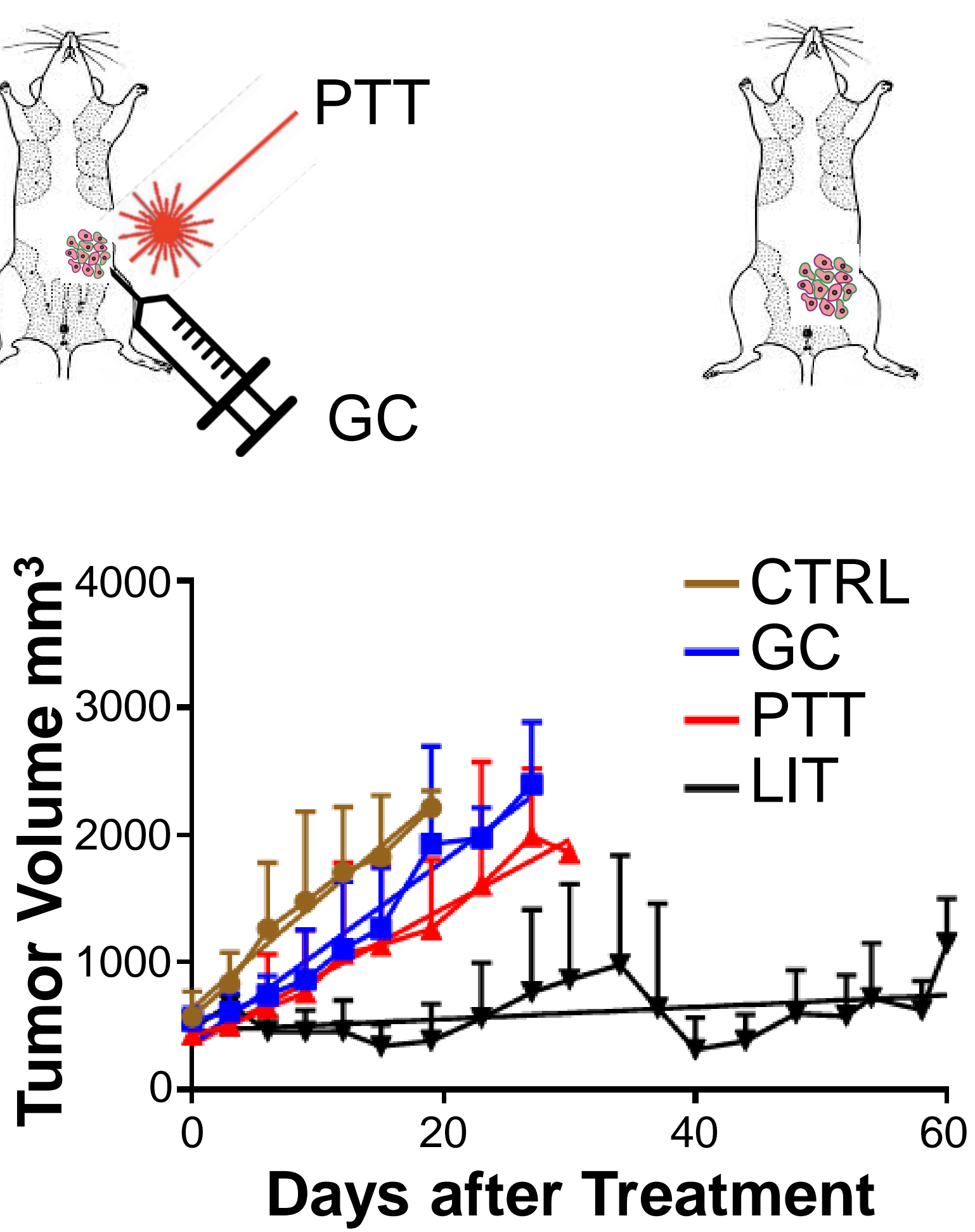

LIT

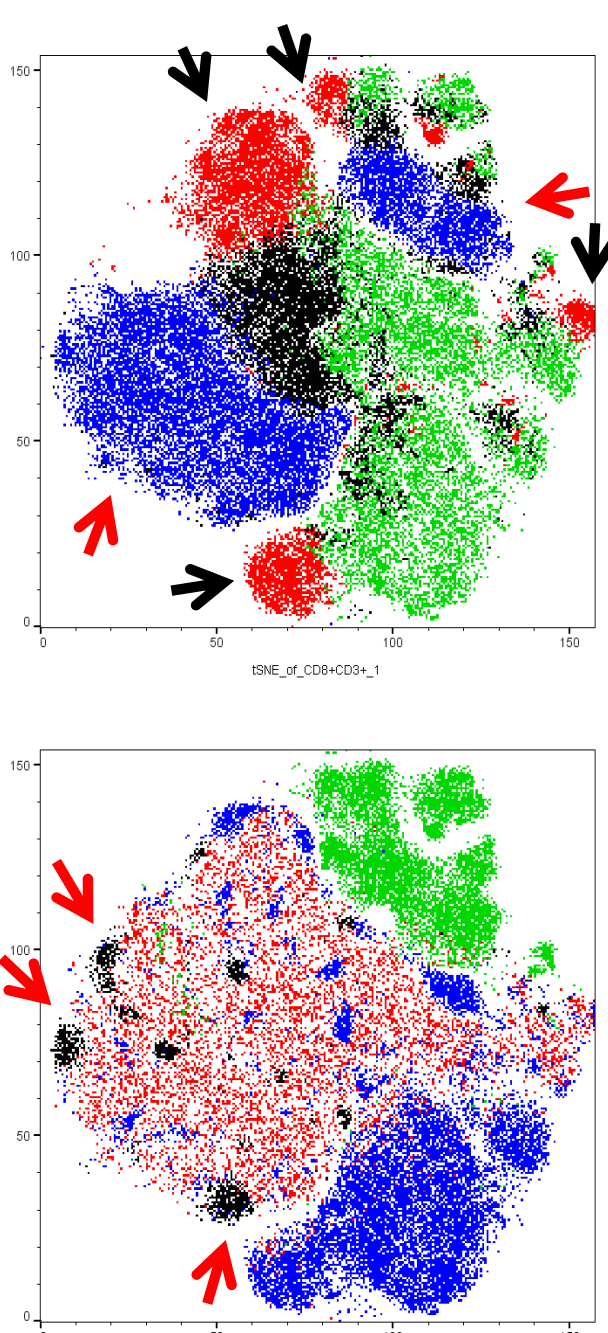

ICD28+CD103+

ICD28+CD69-

ICD28+CD69+

CD28-CD69+

CD44HighCD62L+

CD62L+

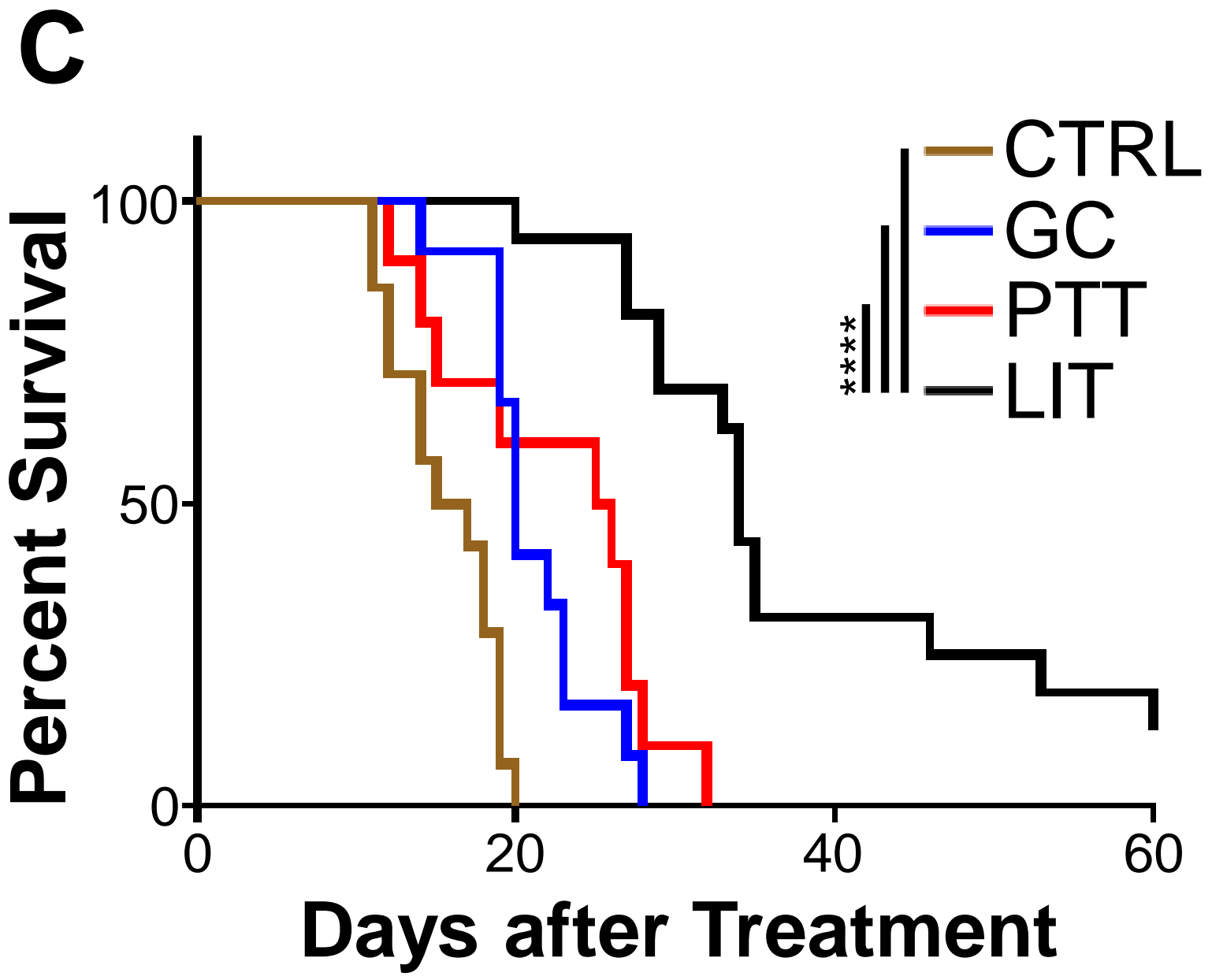

F

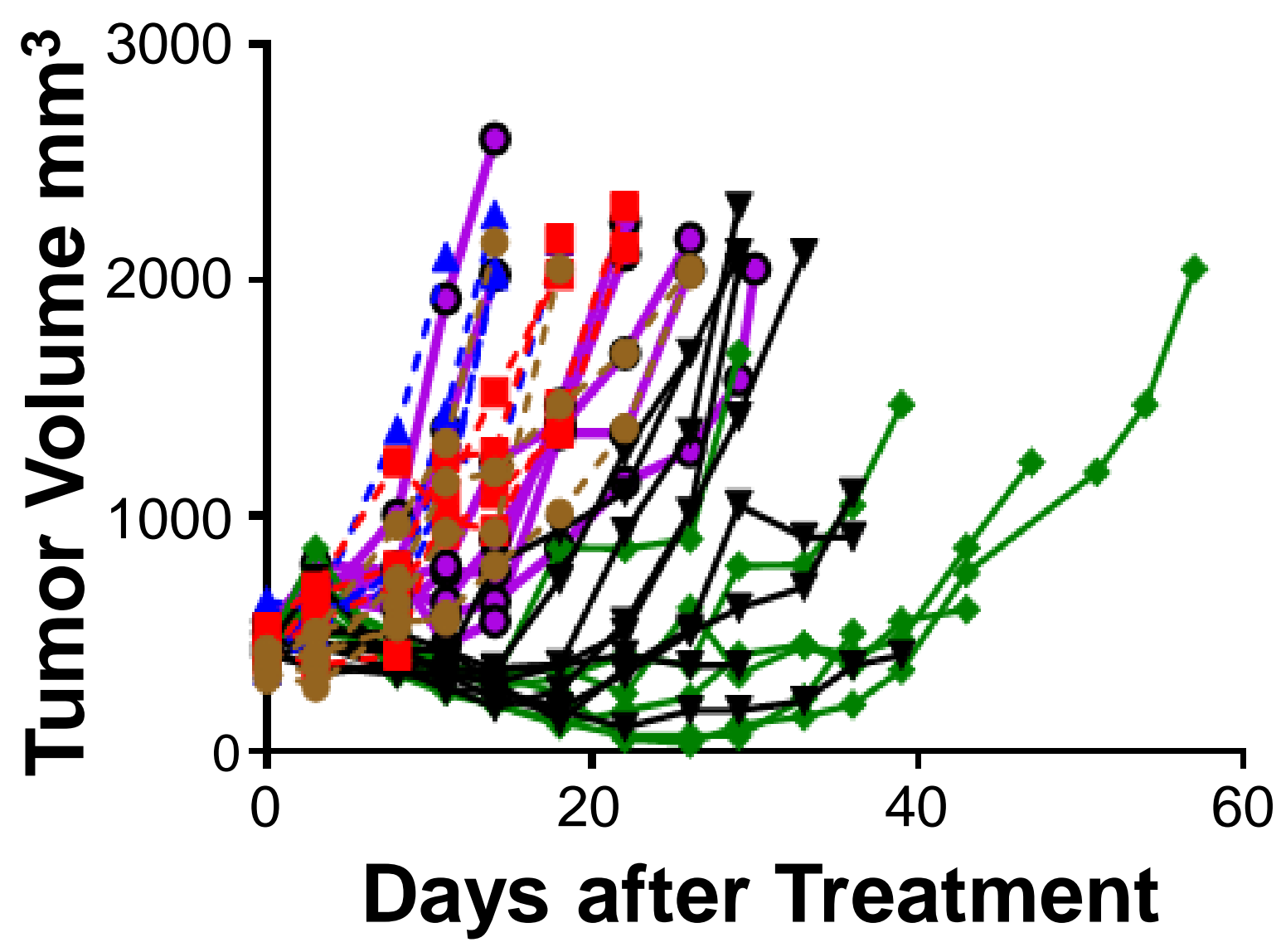

G

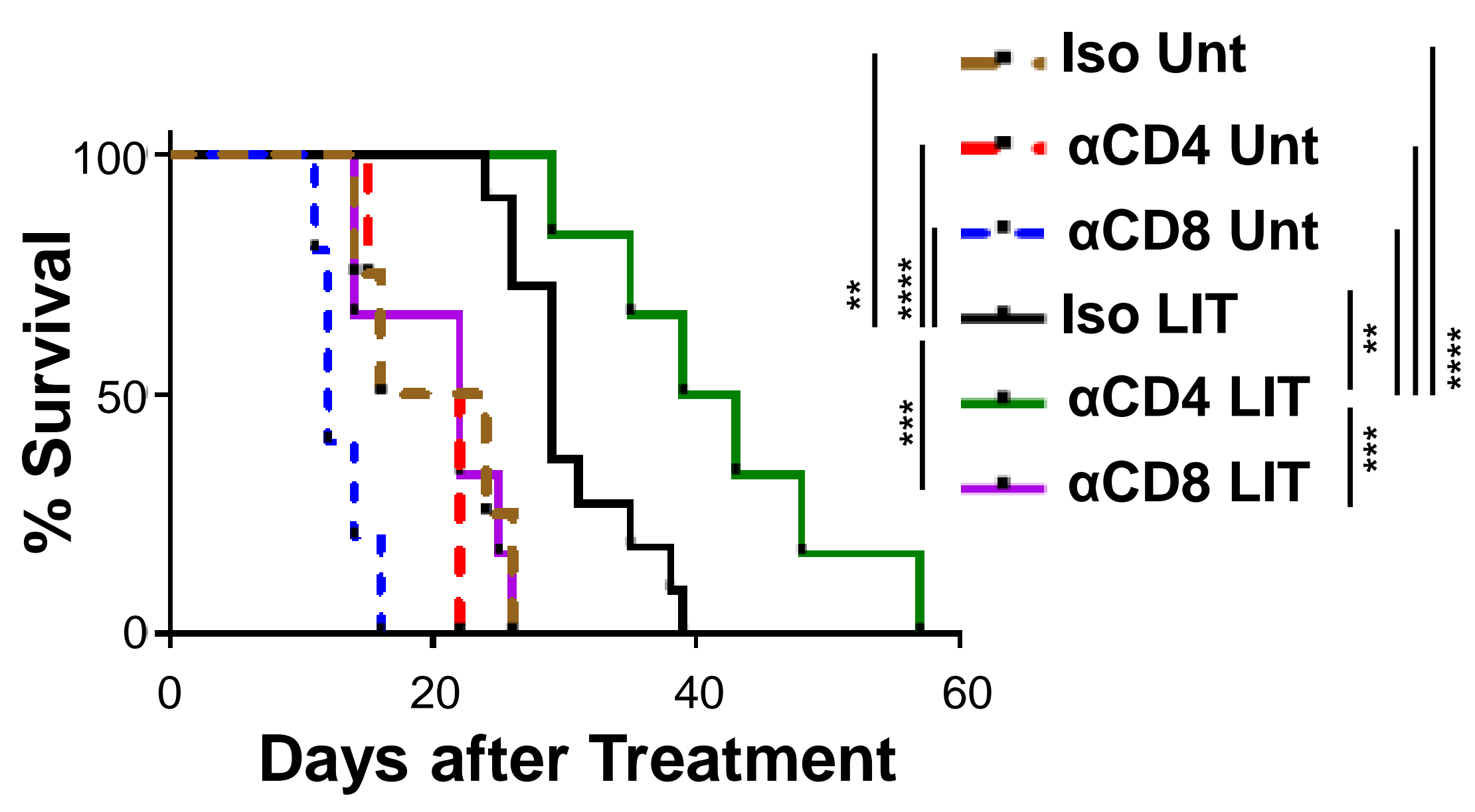

Figure 1. 
A

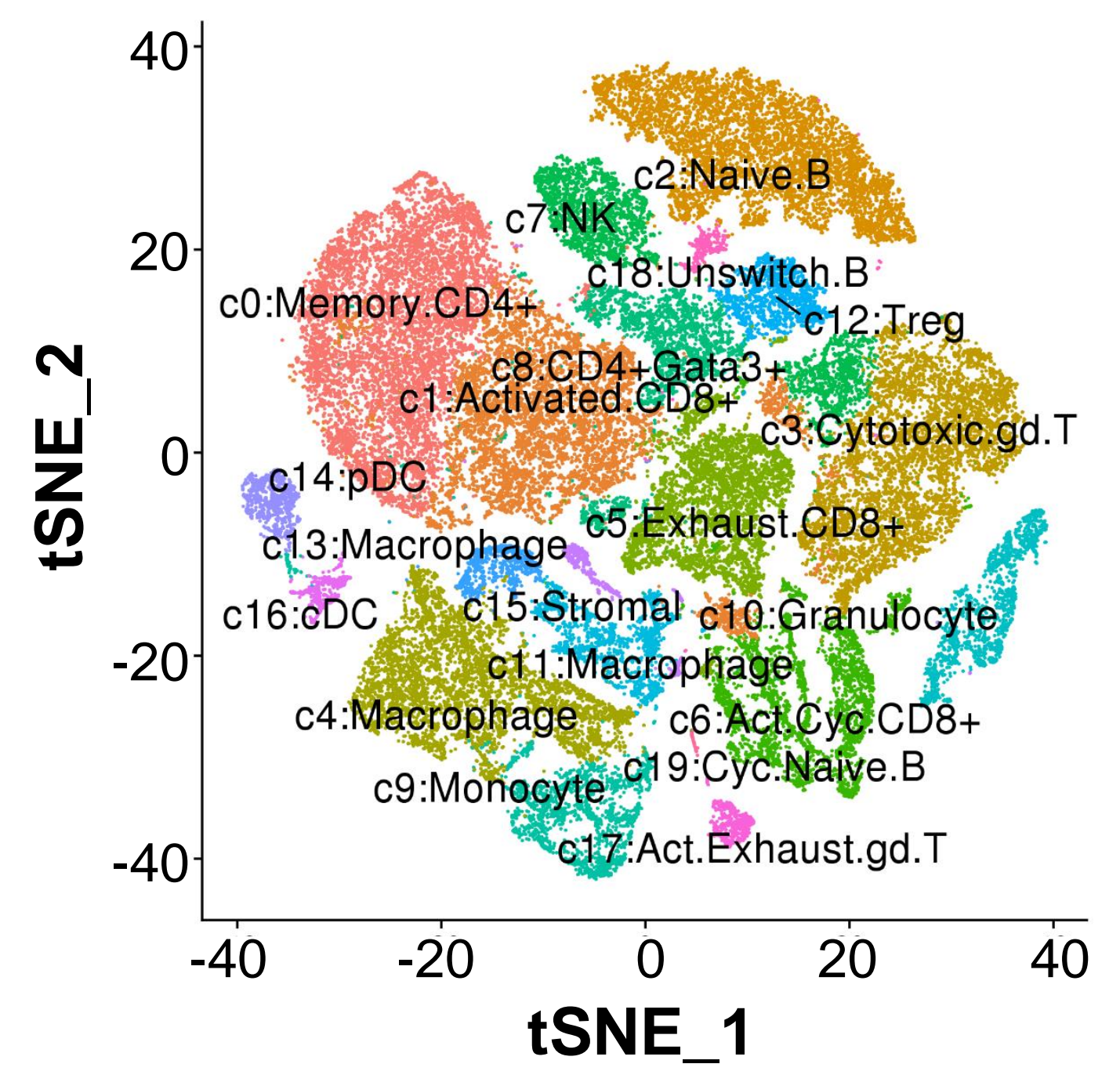

B

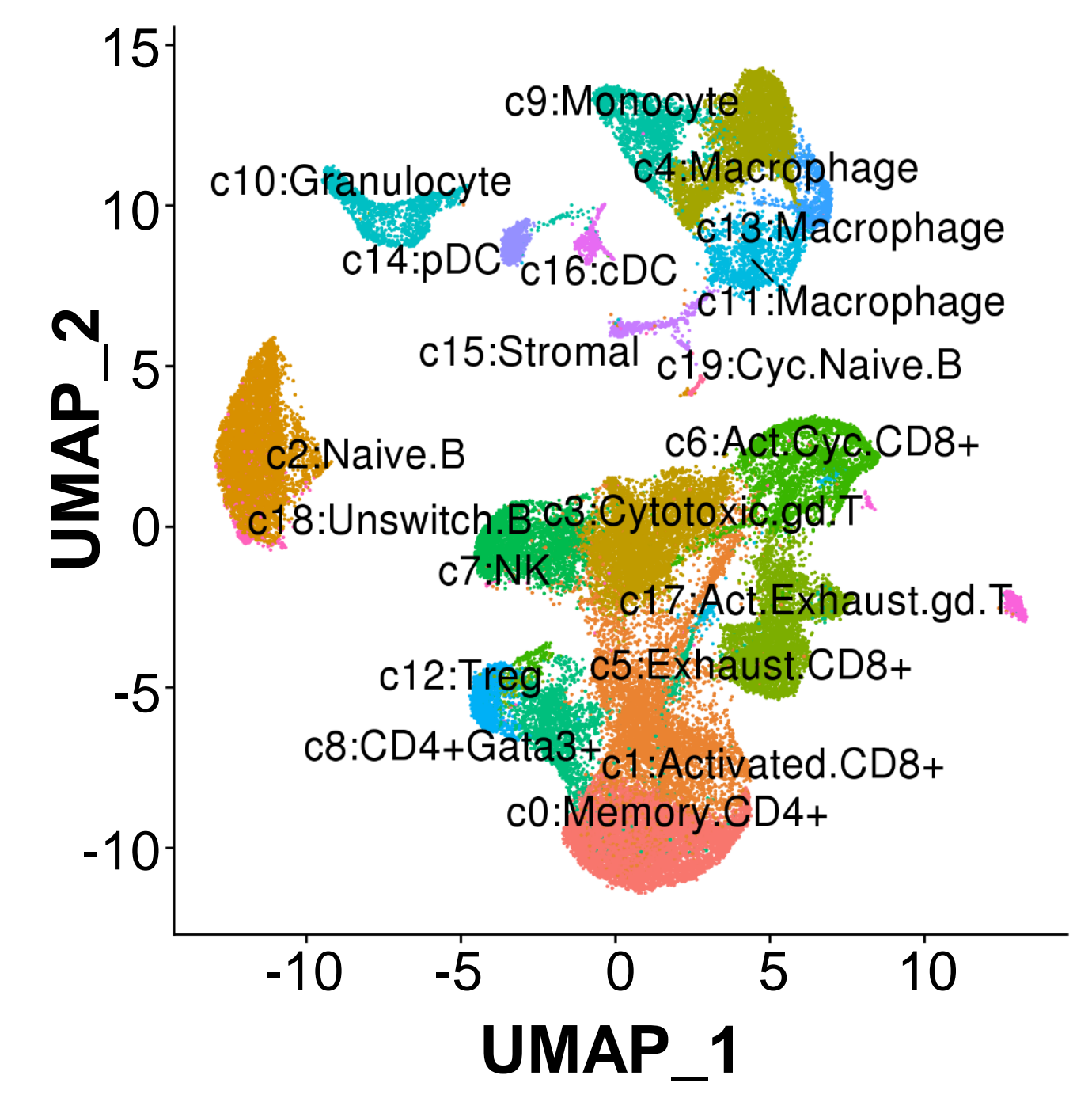

C

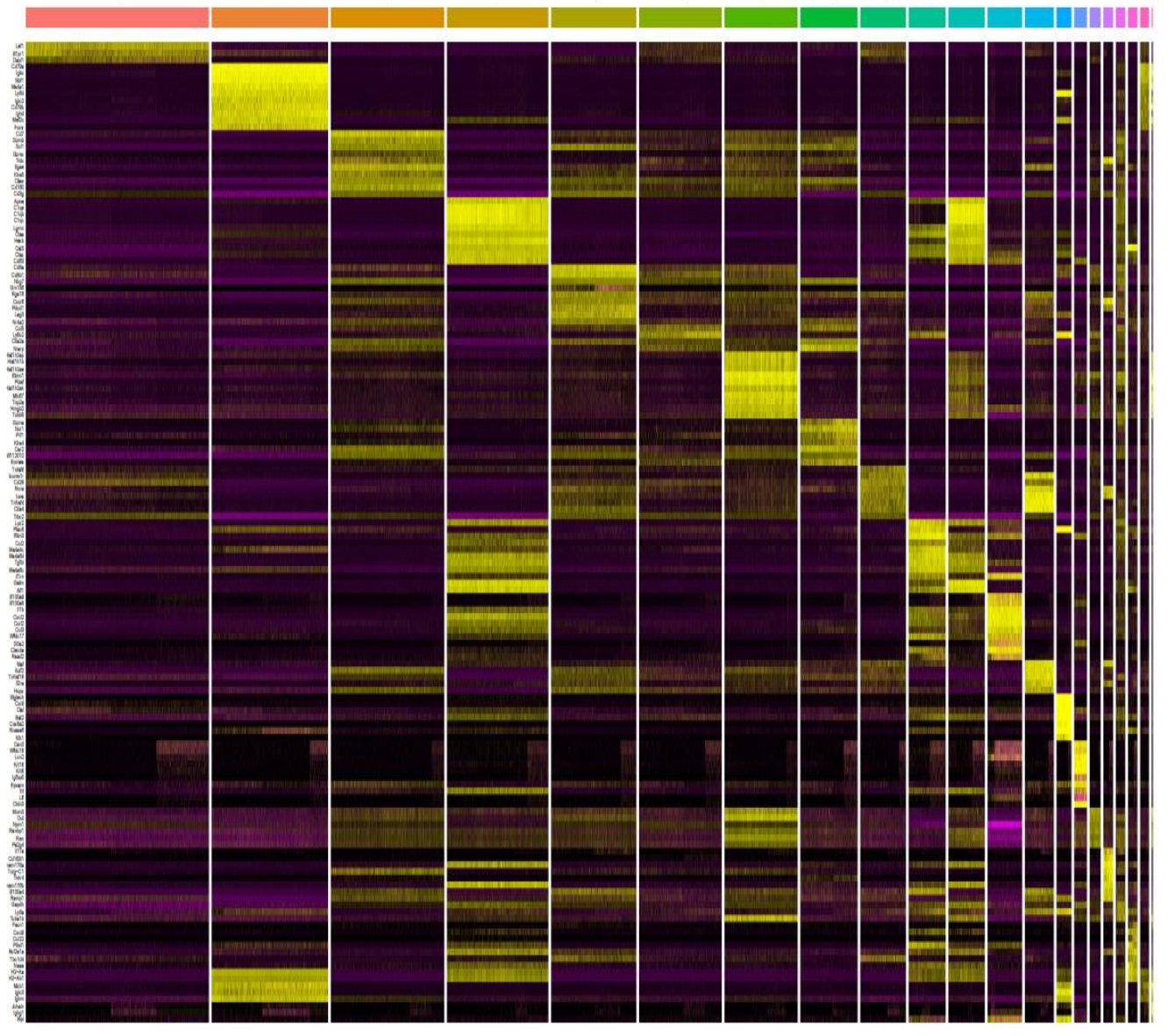

D

C1: Activated CD8
C6: Act. Cyc. CD8

C5: Exhausted CD8

$\mathrm{CO}$ : Memory $\mathrm{CD}^{+}$

C8: CD4+Gata3+

$$
\text { C12: Treg. }
$$
C7: NK.
C10: Granulocyte-
C9: Monocyte

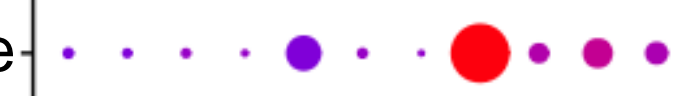

C13: Macrophage . . . . . . .

C11: Macrophage $\ldots$

C16: $\mathrm{CDC}$ -

C2: Naïve $B$ cell-

C18: Unswitched B cell

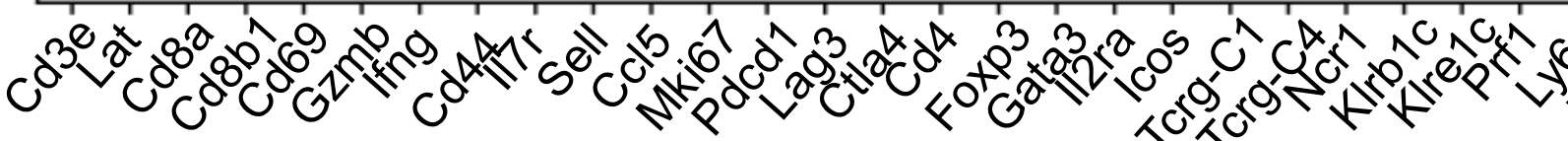

B cell

Average Expression

2
1
0
-1
- Percent Expressed 0
$: \quad 25$
$: 50$
875
100

\section{E}

\section{Genes}
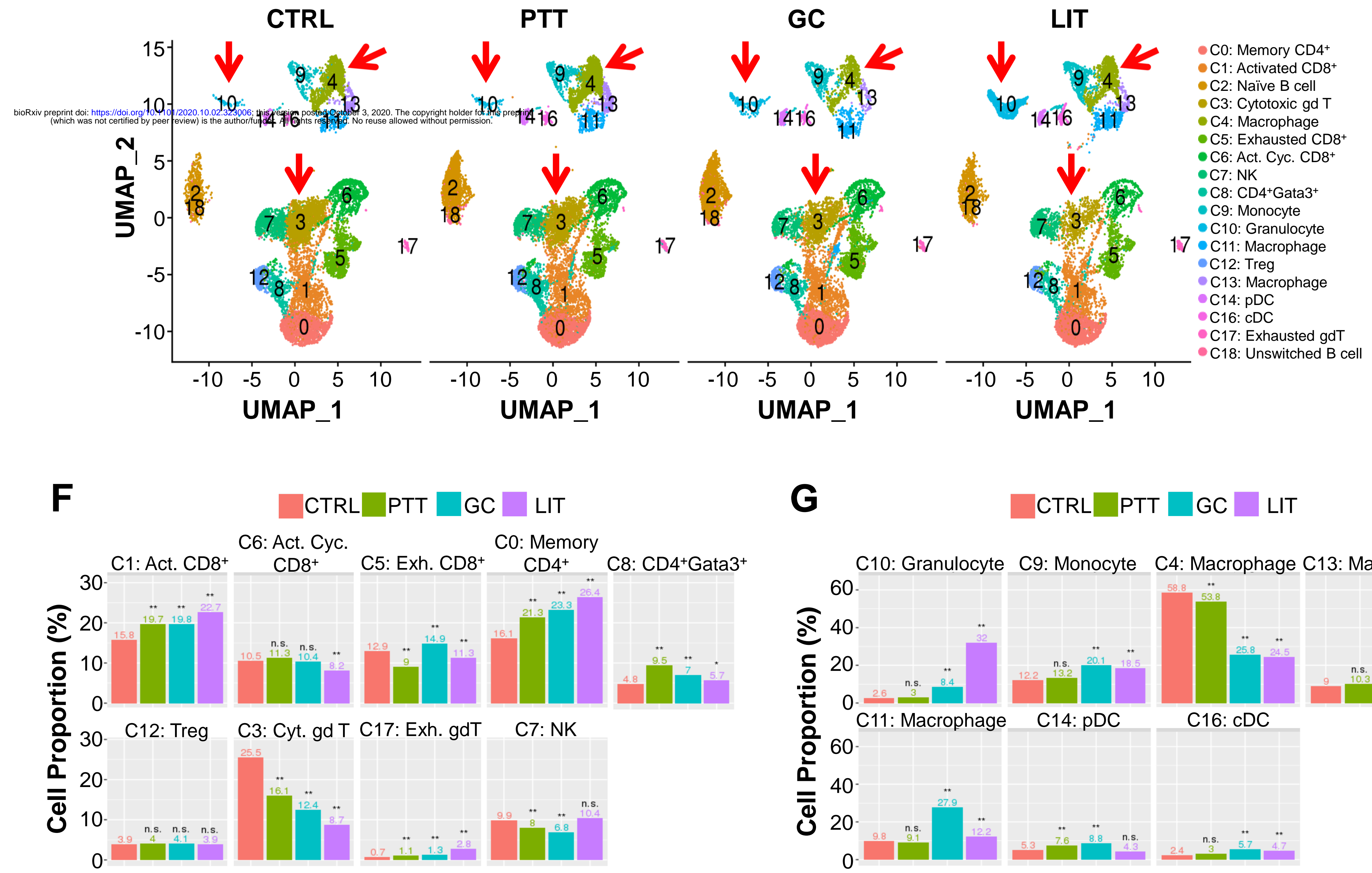

G

CTRL $\square$ PTT —GC — LIT

Figure 2.

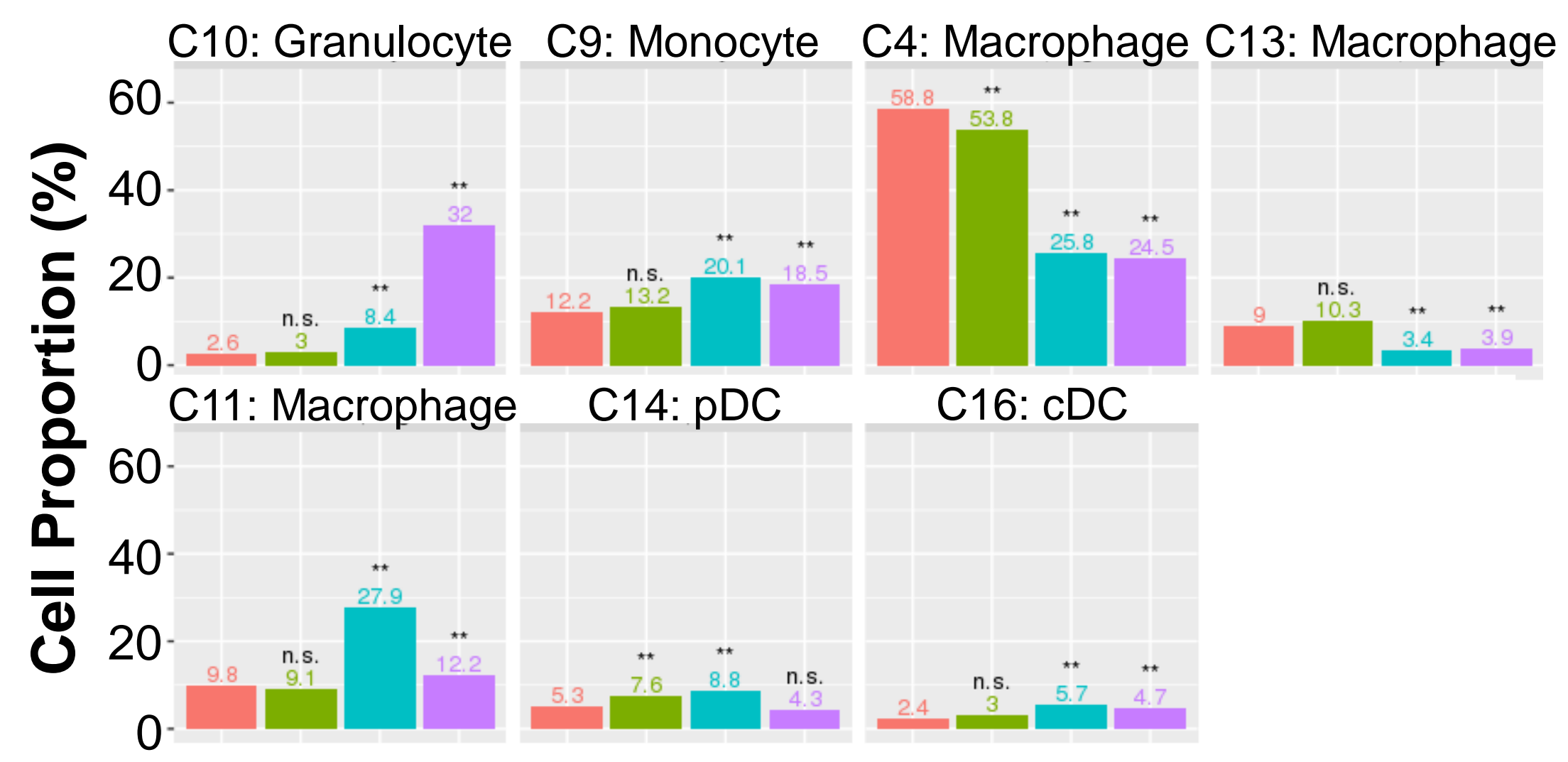


A

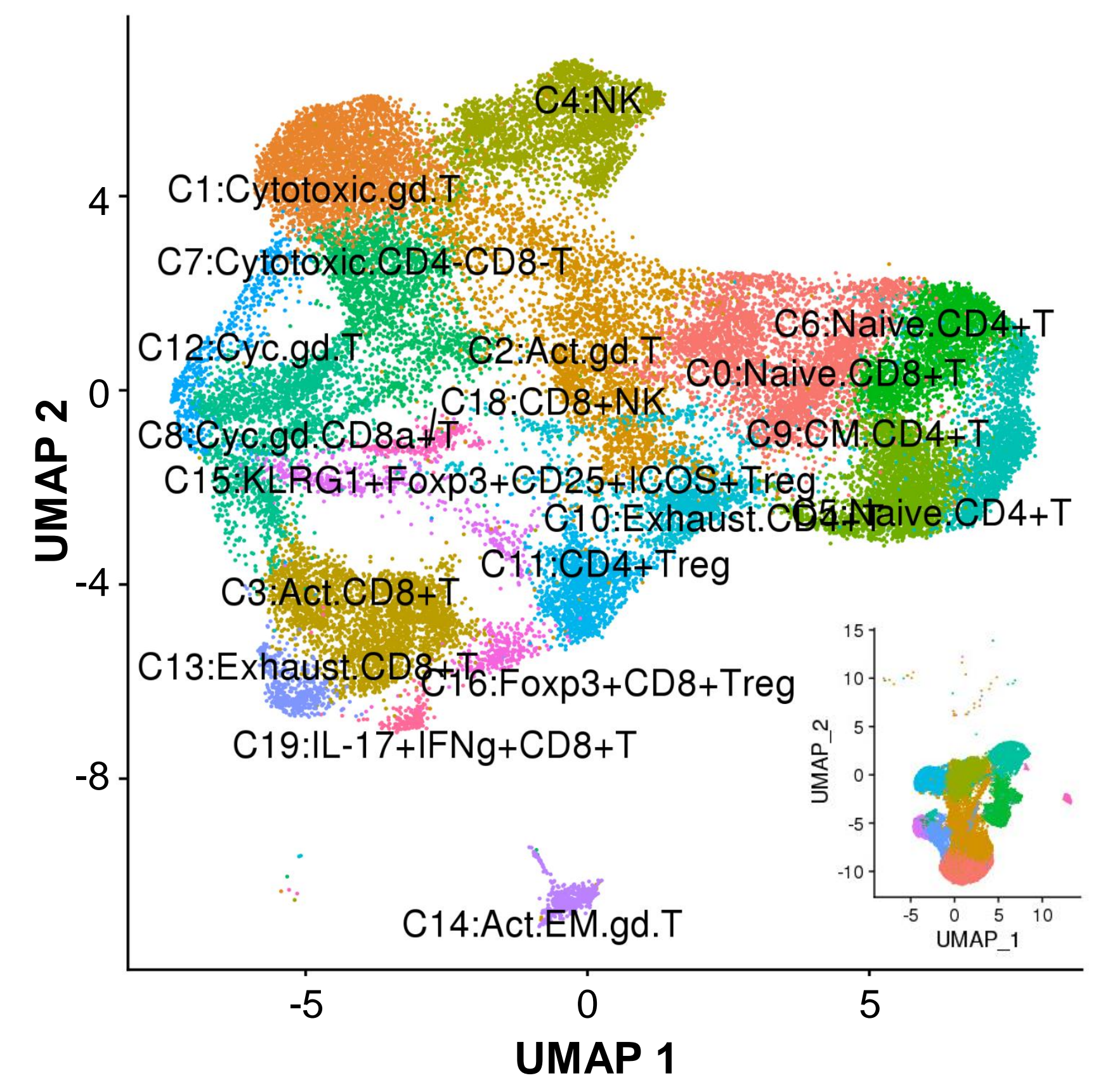

C

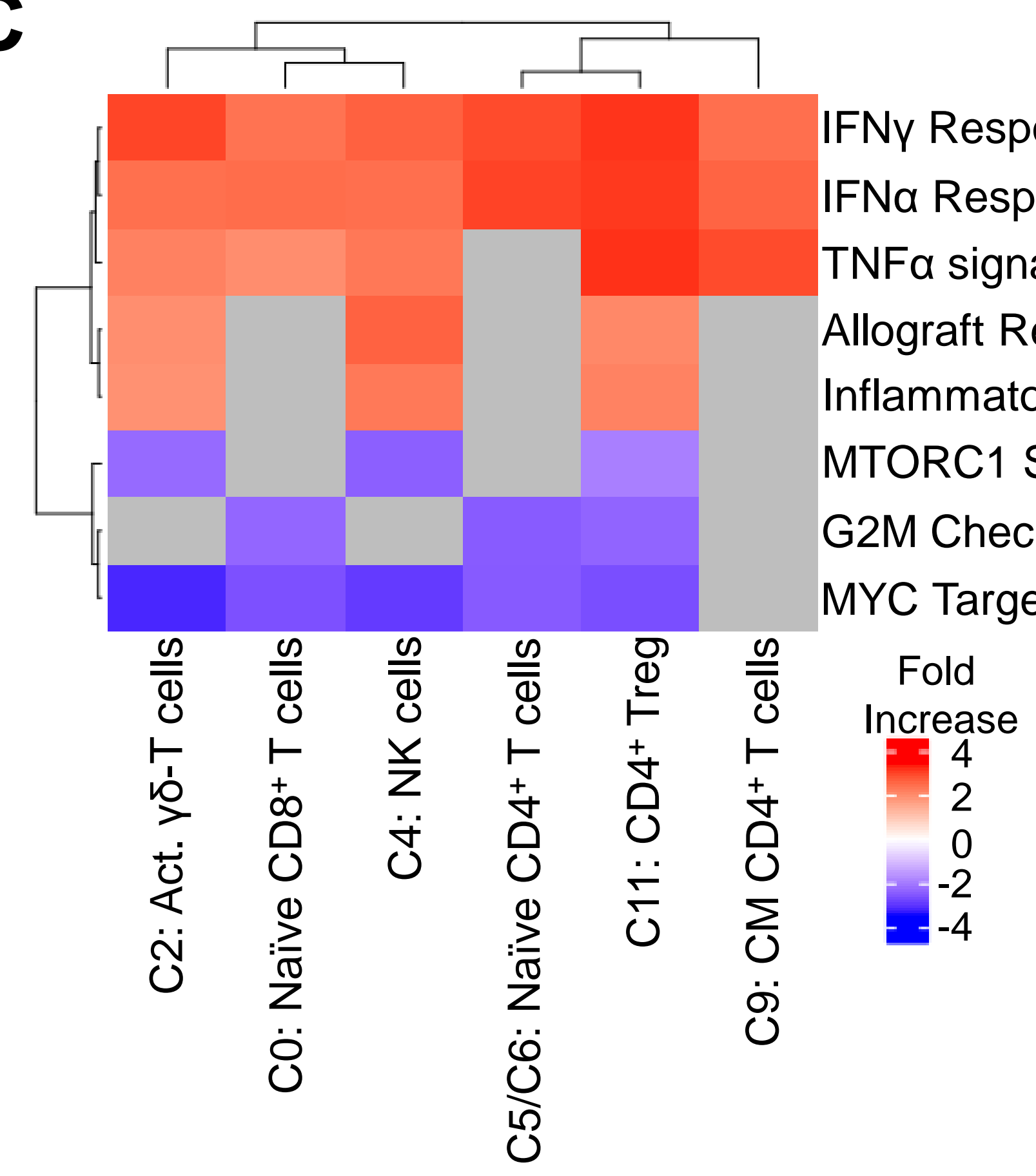

D

\section{LIT vs CTRL}

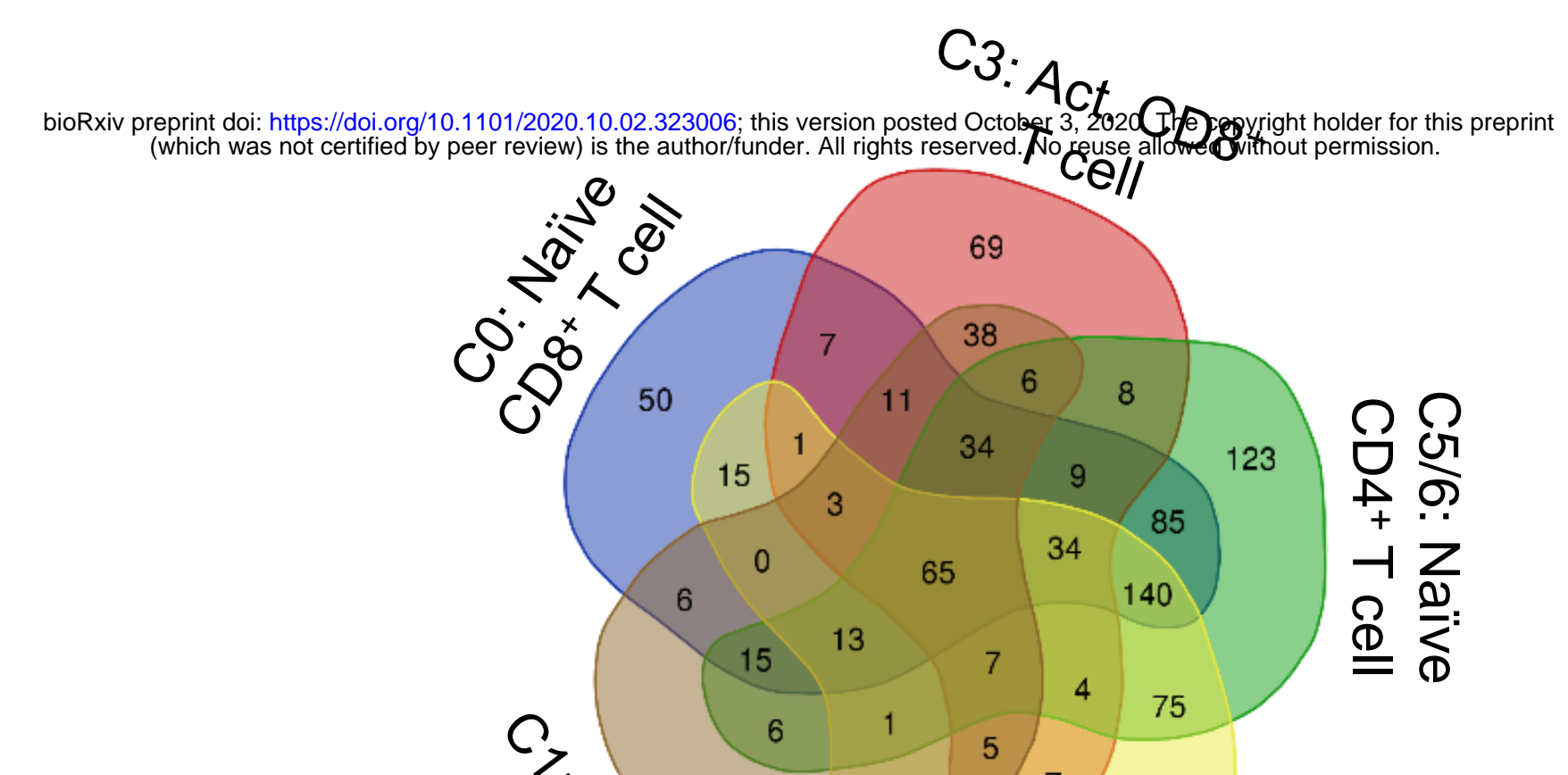

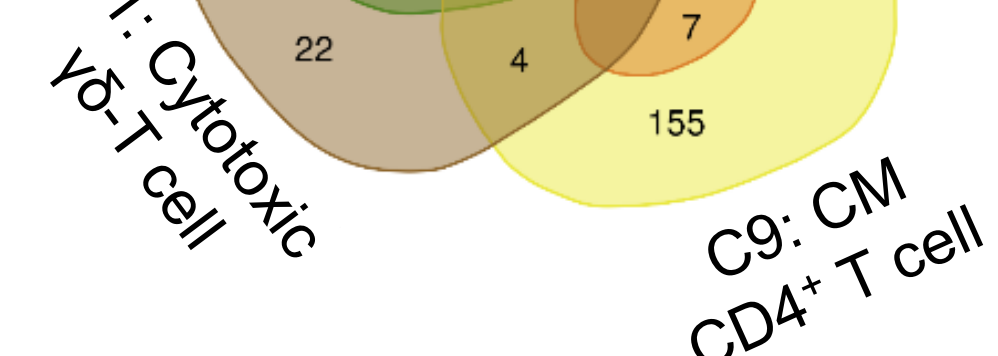

LIT vs PTT

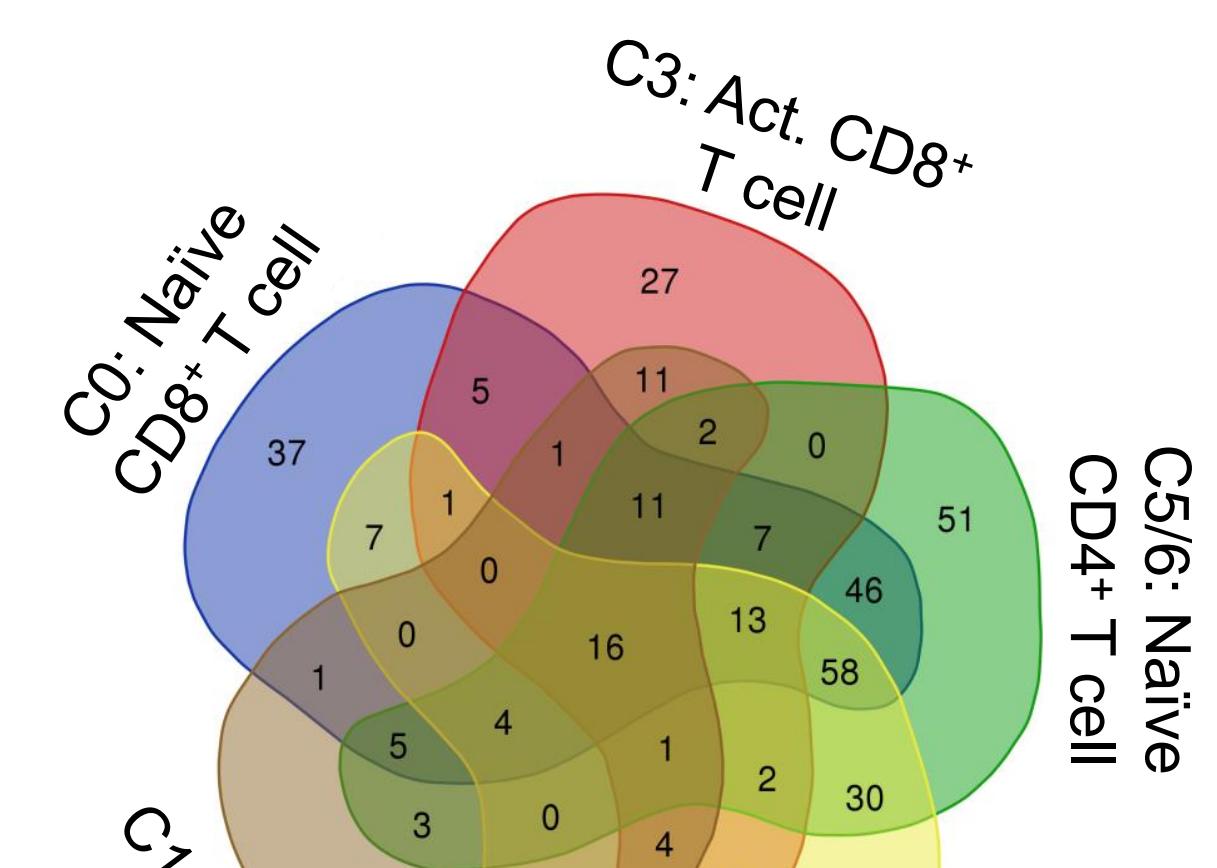

27
B

C0: Naive C3: Act.

CTRL PTT $\triangle$ GC $₫$ LIT

C13: Exh. C16: C19: IL17/IFNy C5: Naive C6:Naive CD8 ${ }^{+}$cell $\mathrm{CD}^{+} \mathrm{T}$ cells $\mathrm{CD}^{+}$Treg $\mathrm{CD}^{+} \mathrm{T}$ cell $\mathrm{CD}^{+} \mathrm{T}$ cell $\mathrm{CD}^{+} \mathrm{T}$ cell

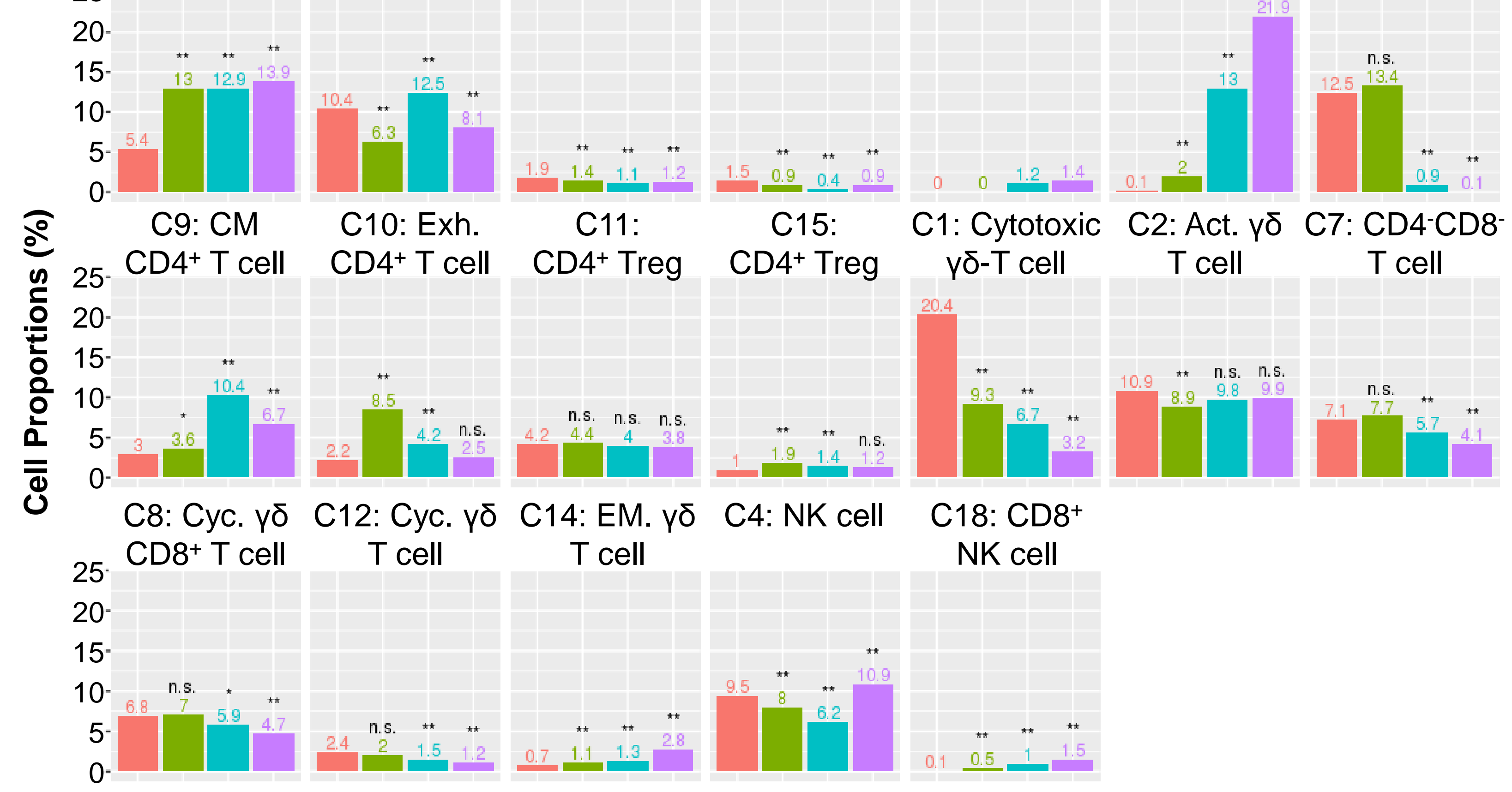

E

CTRL PTT GC LIT

Fos

C0:

Naive.CD8+T
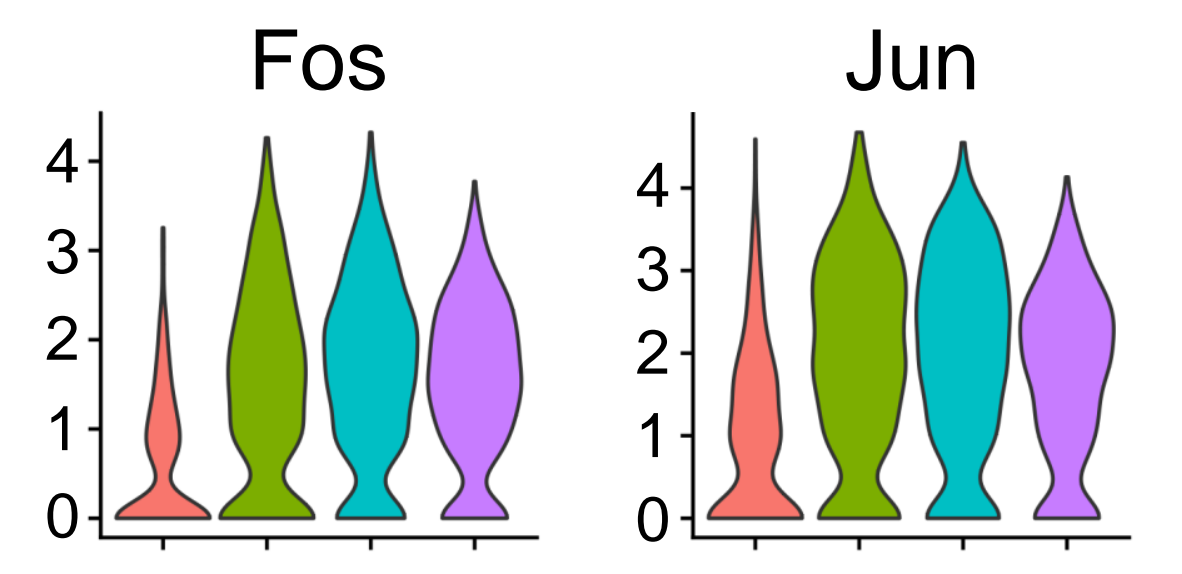

Ptprcap
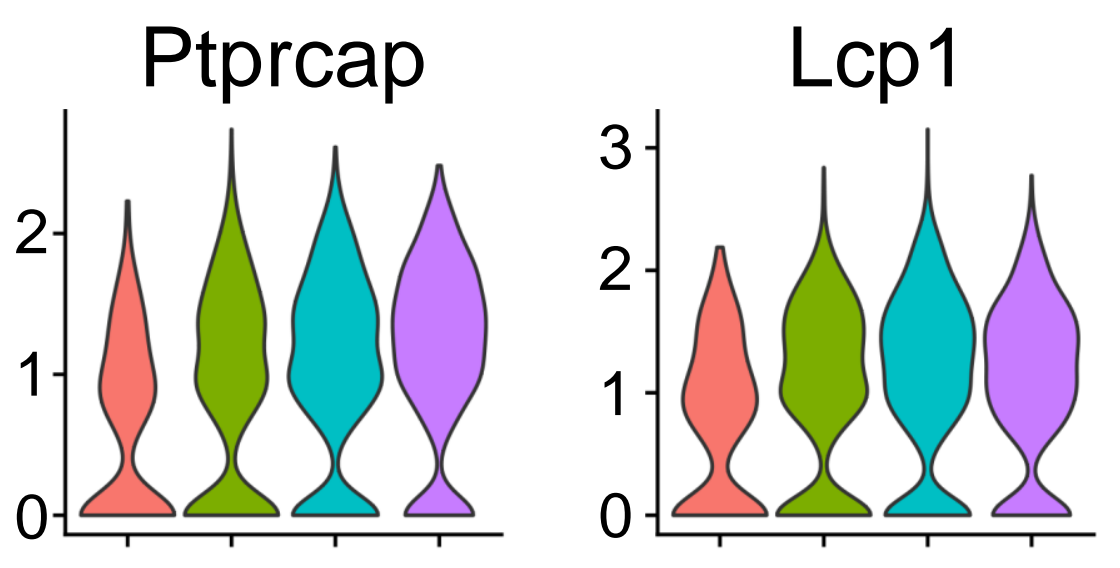

C3: Act.CD8+T
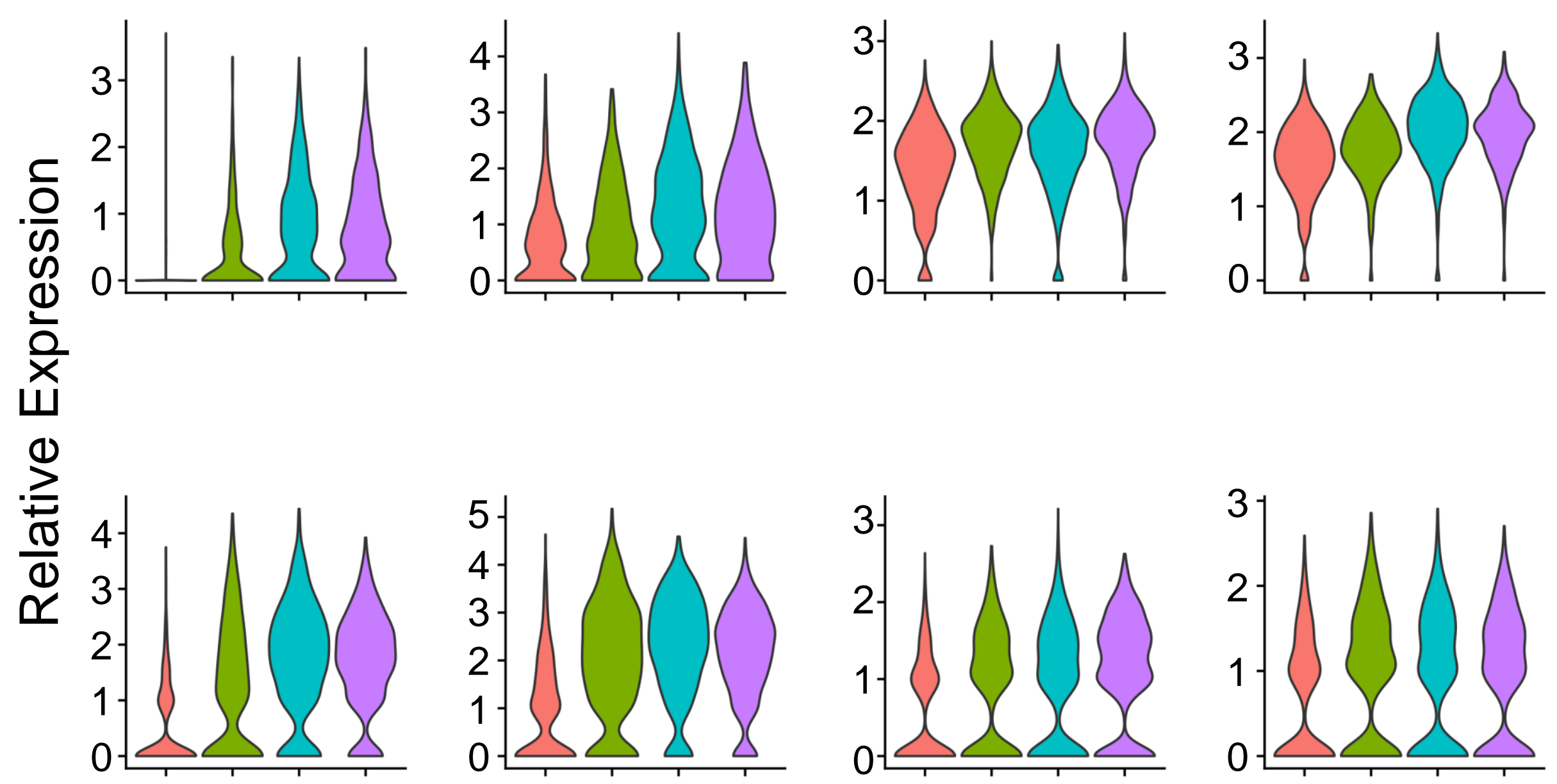

C5\&6:
Naive.CD4+T
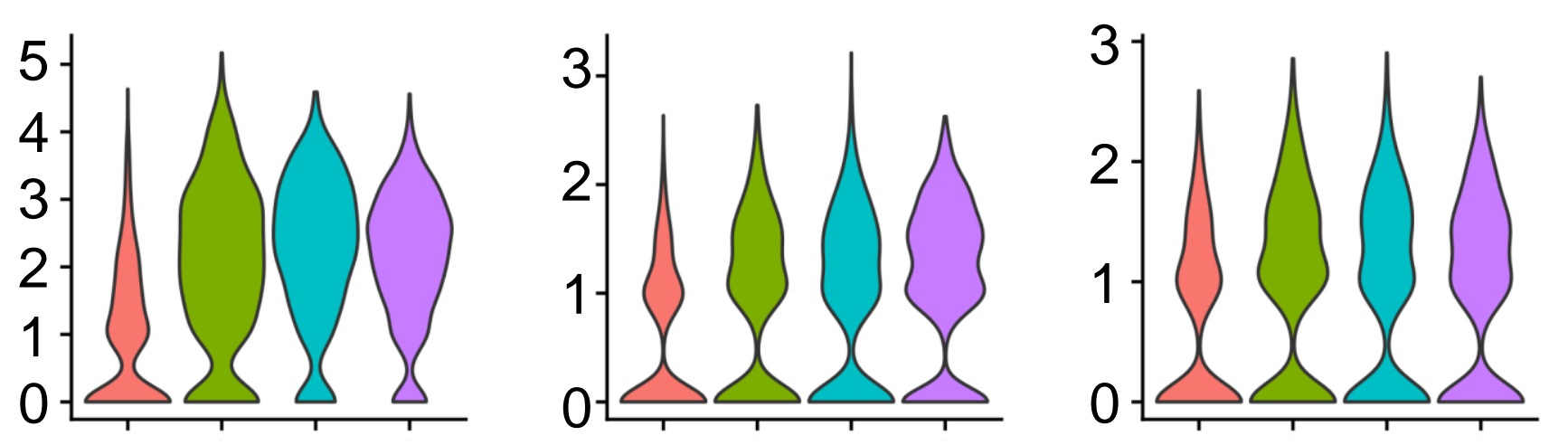

C9:

CM.CD4+T
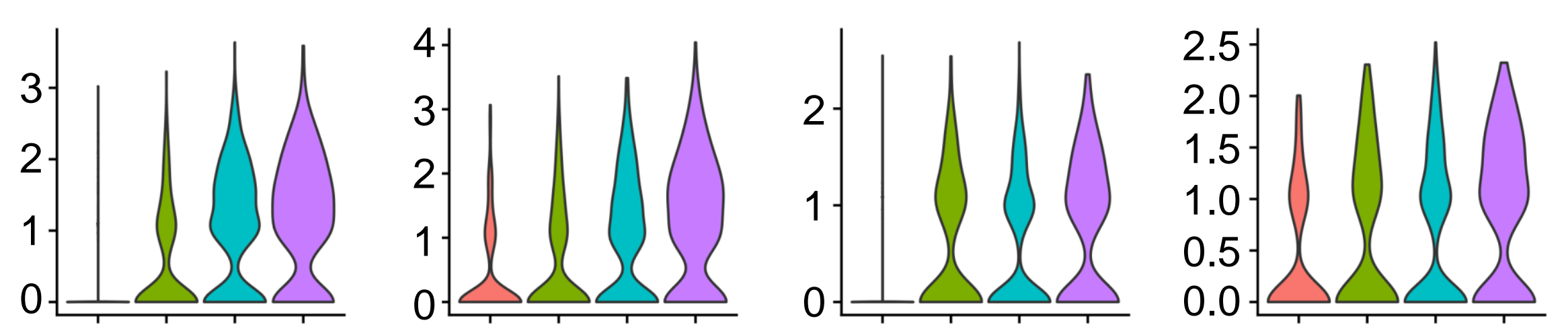

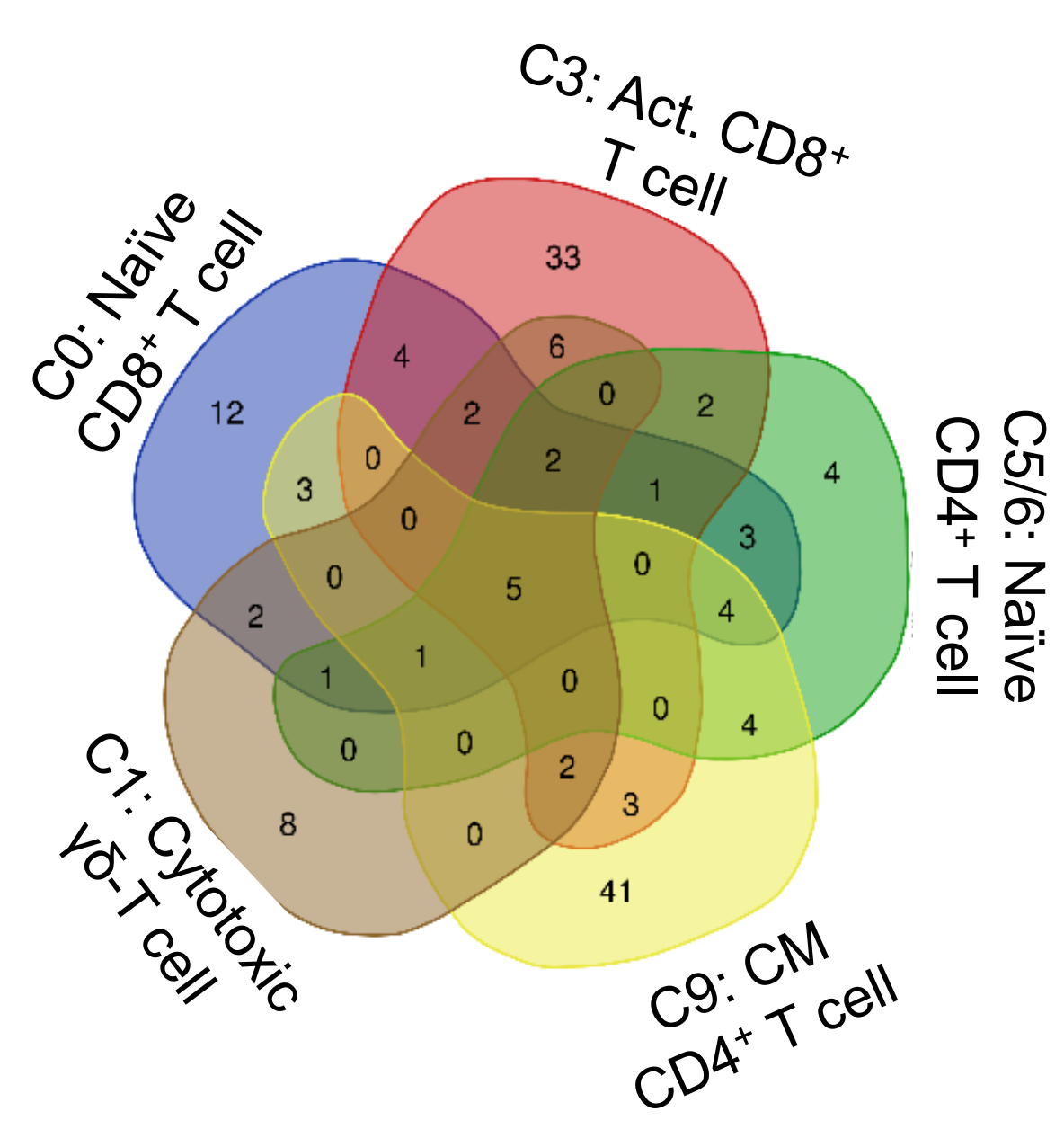



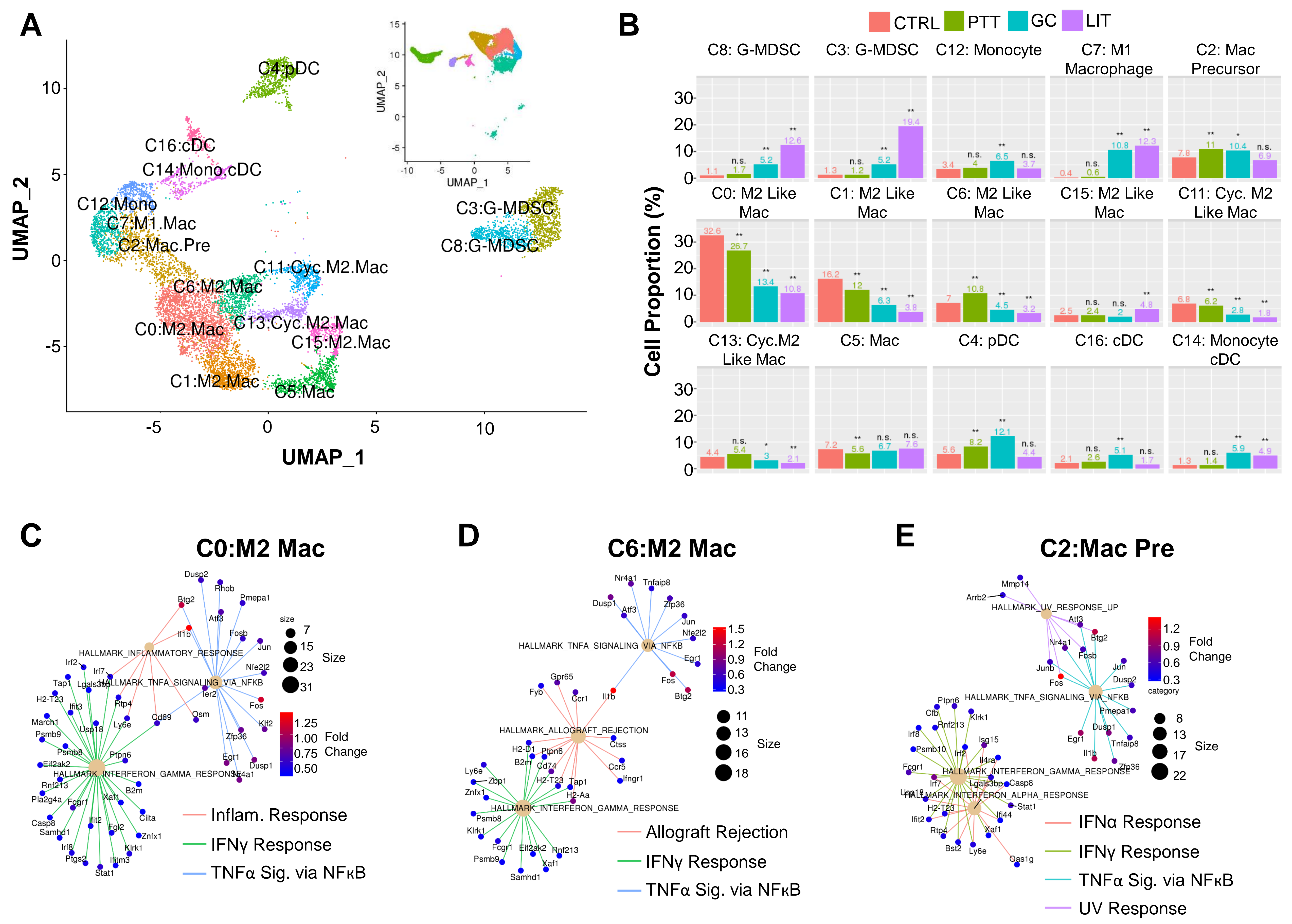

$\mathbf{F}$

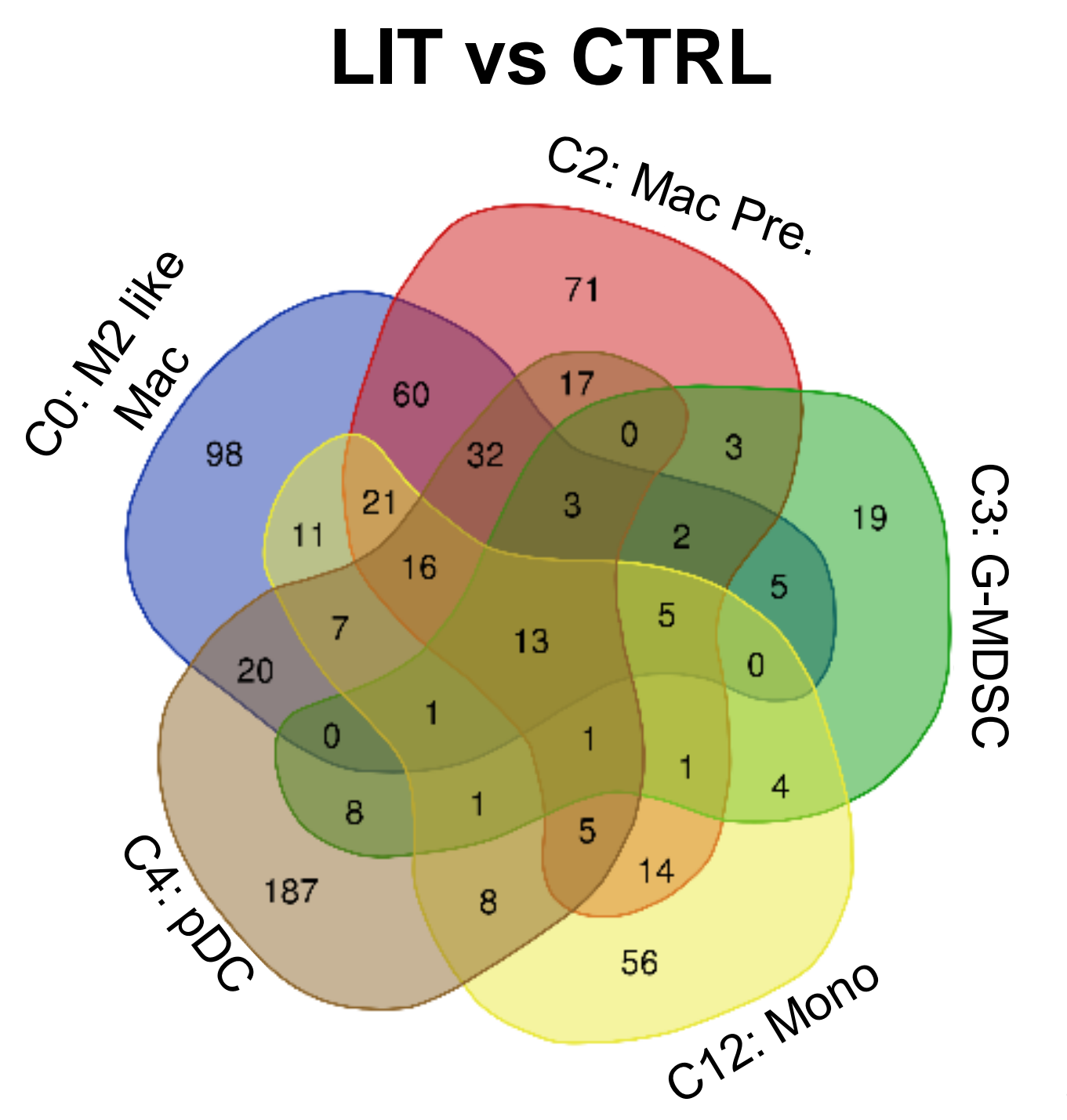

Figure 5.
G
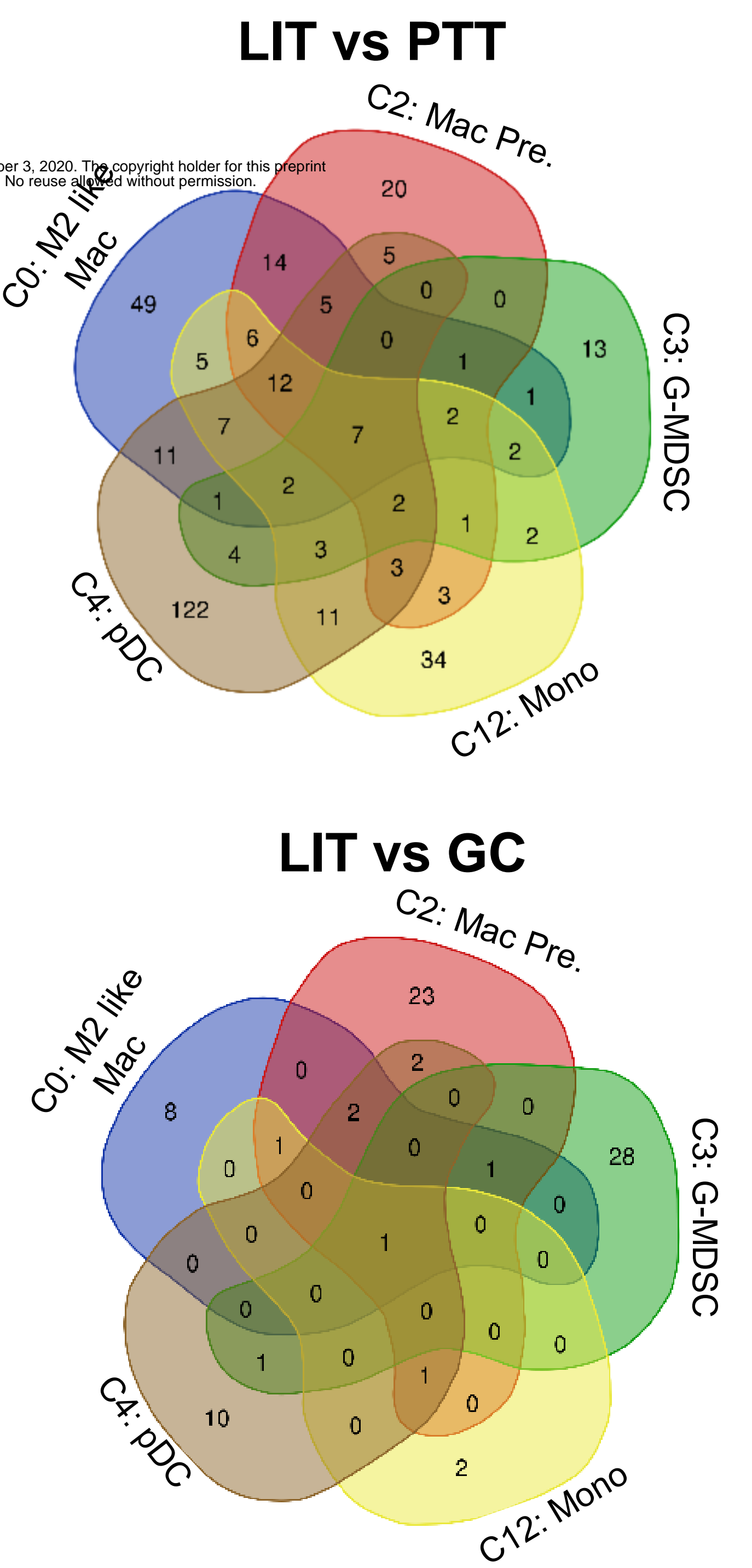

C2:Mac Pre

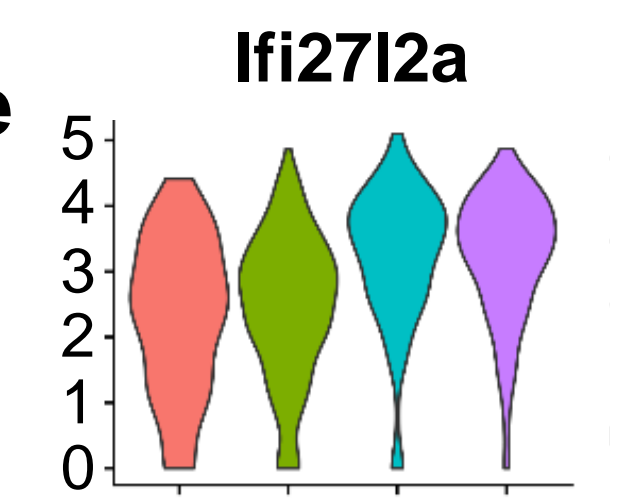

Irf5
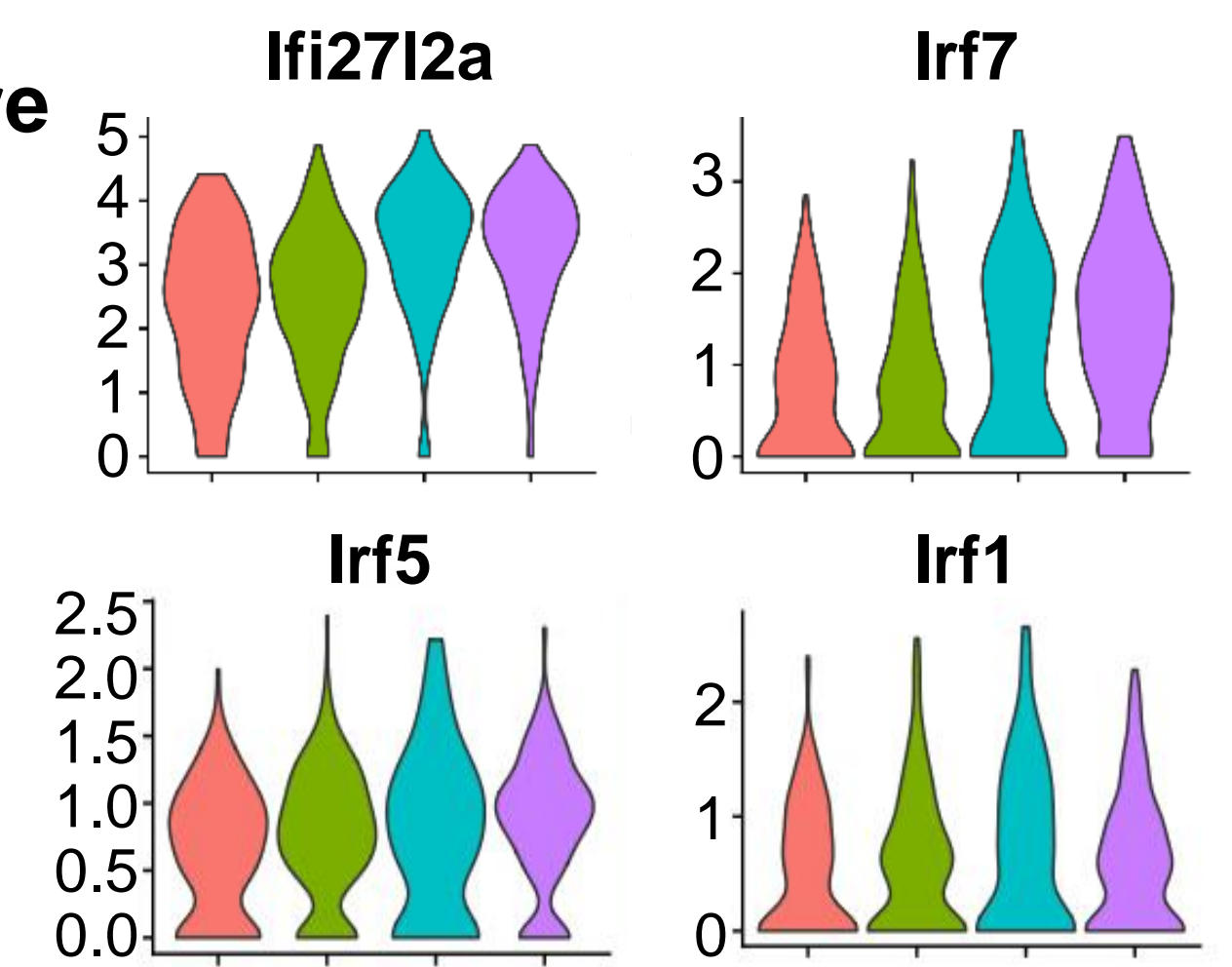

Irf1
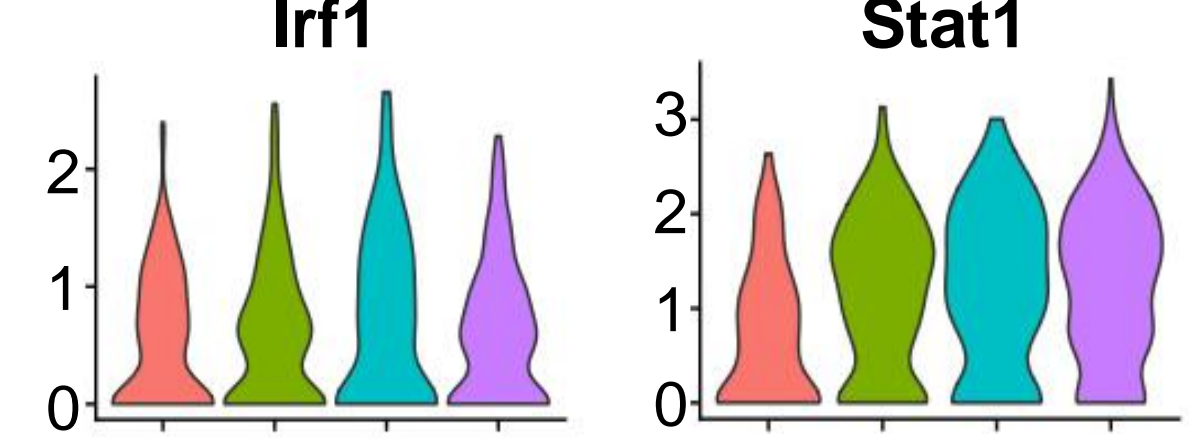

CTRL $\square$ PTT GC LIT

C12:Mono
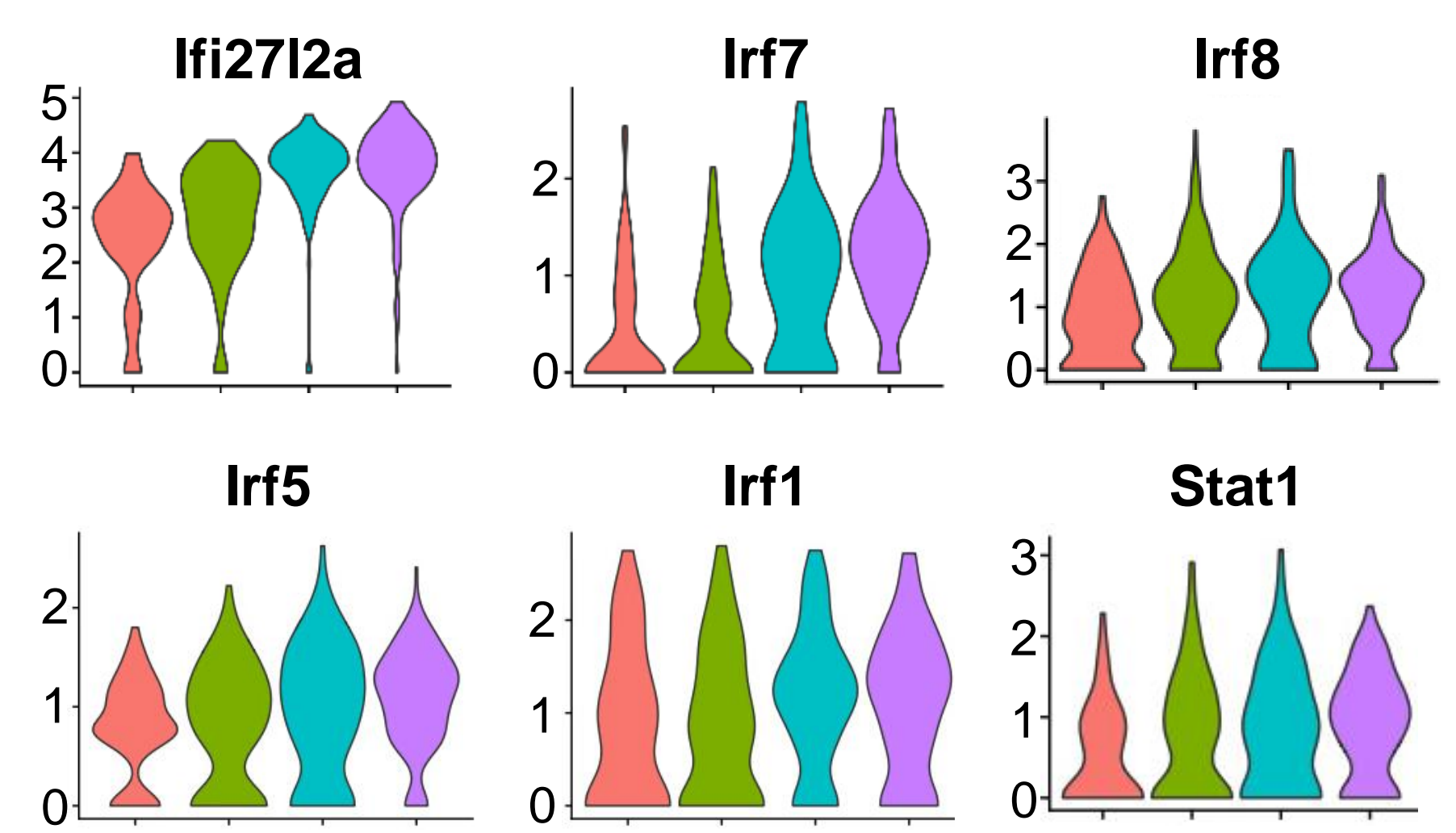

Irf1

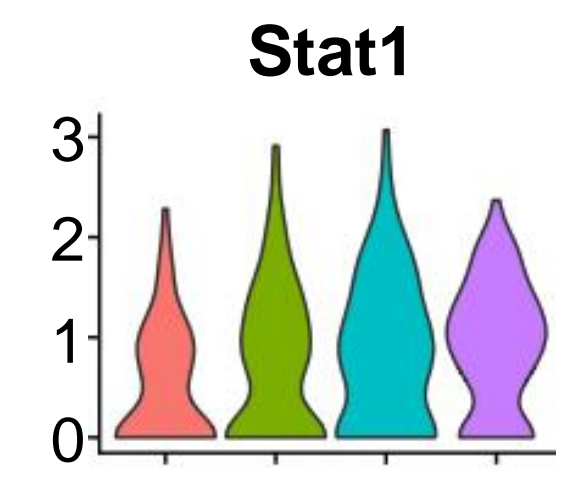


A
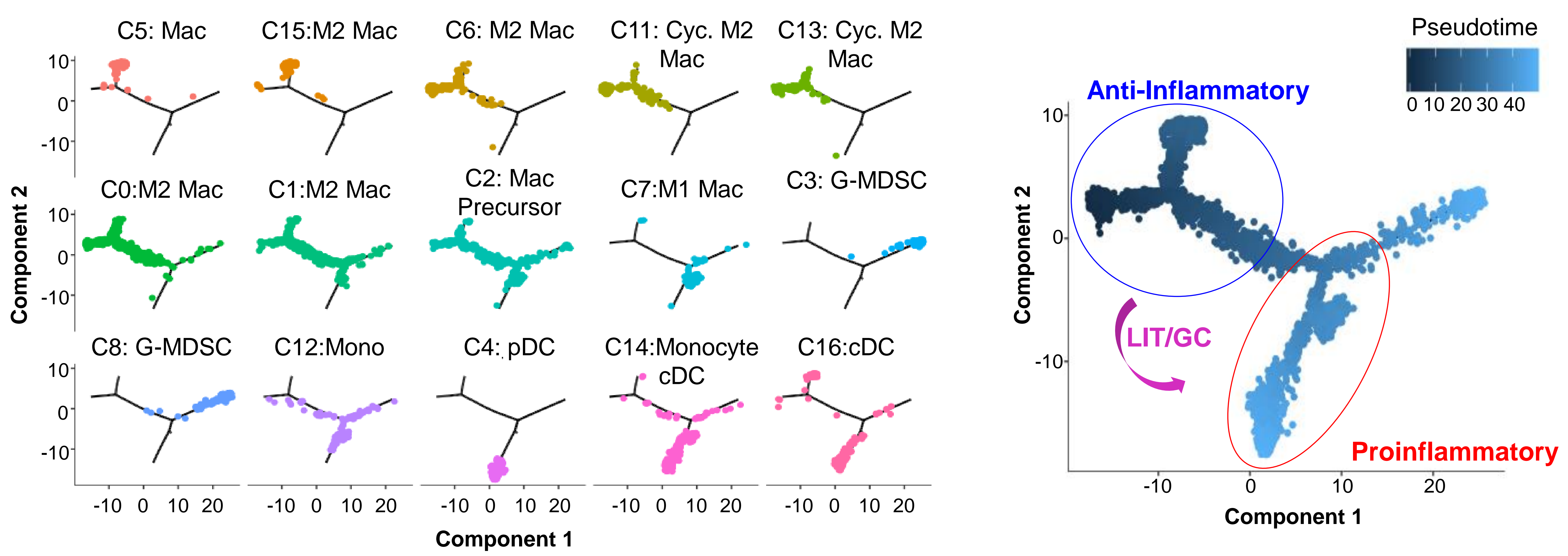

B

\section{Proinflammatory vs Anti-inflammatorv}

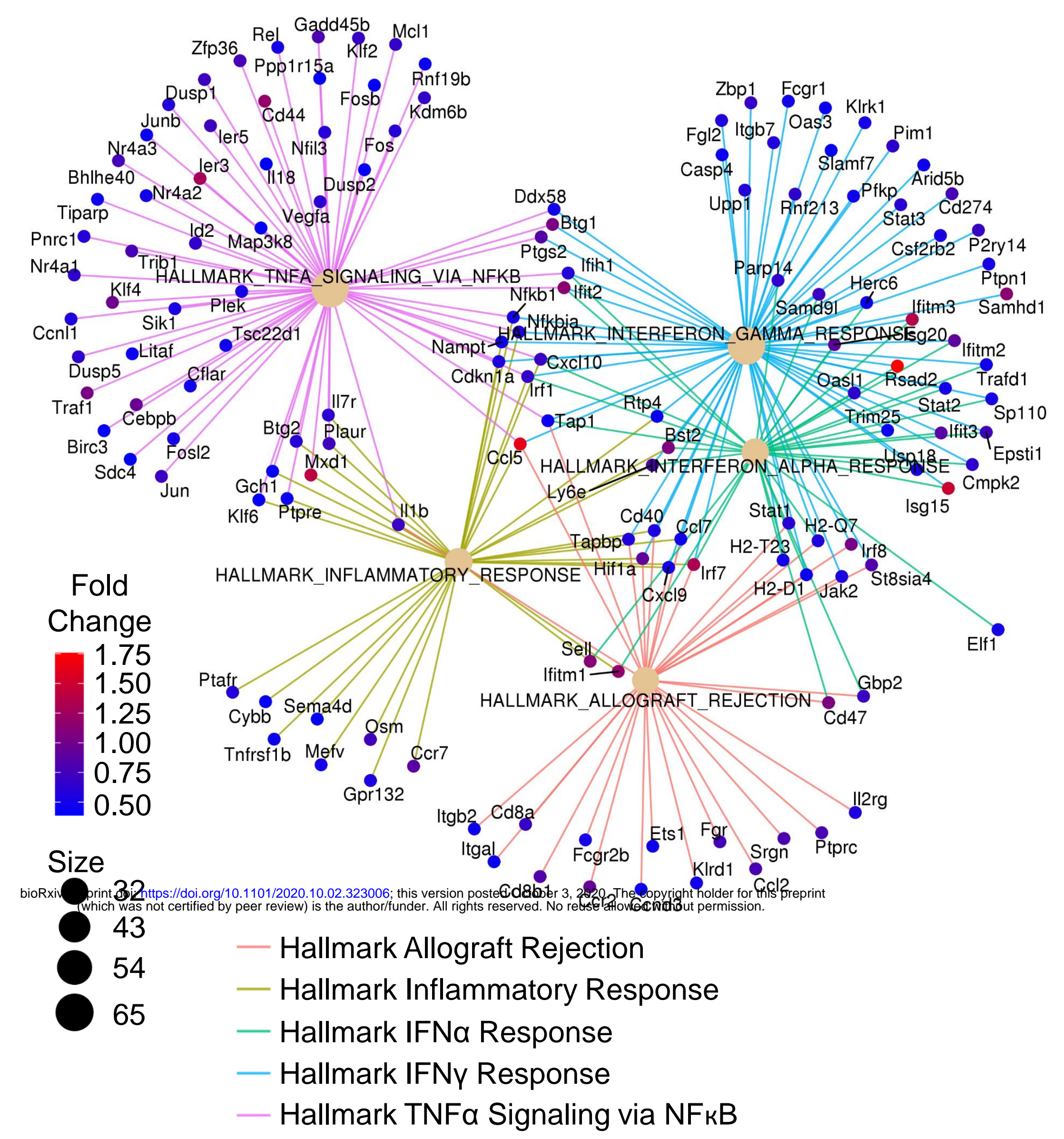

E

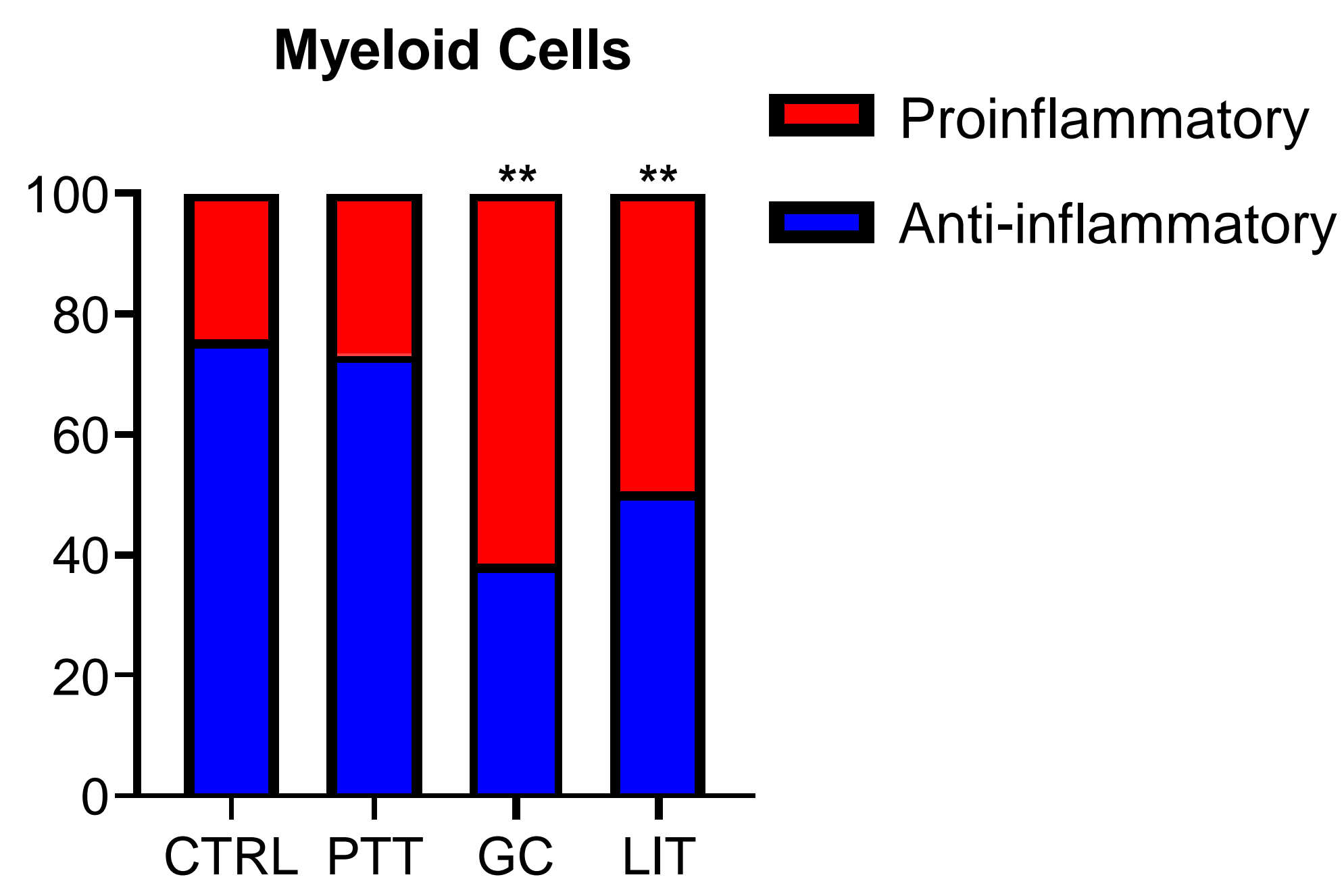

D

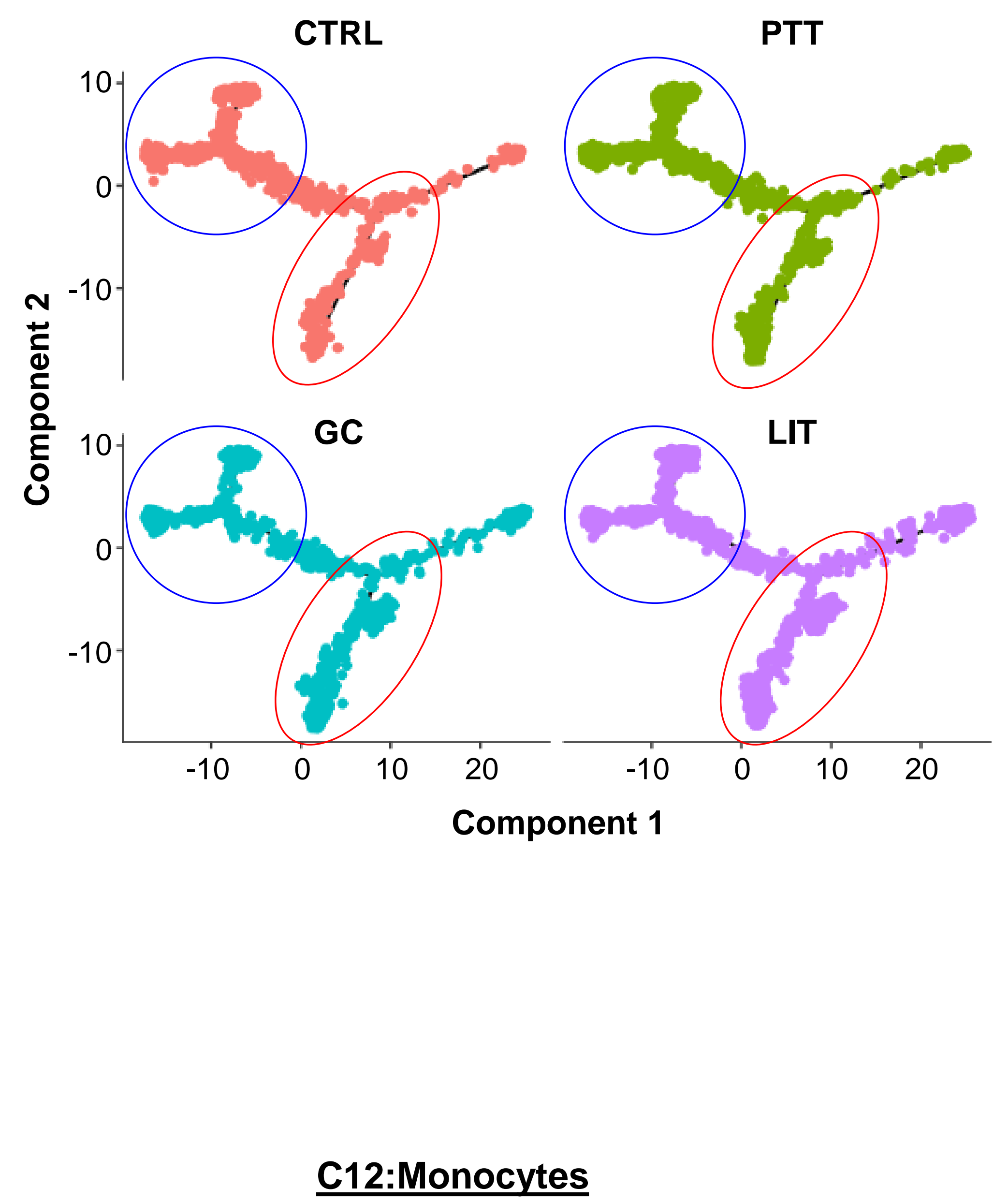

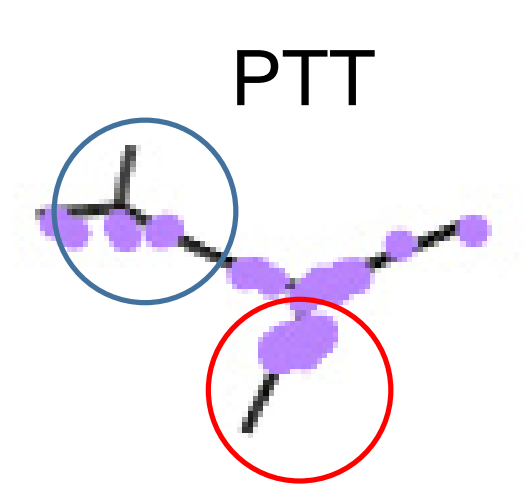
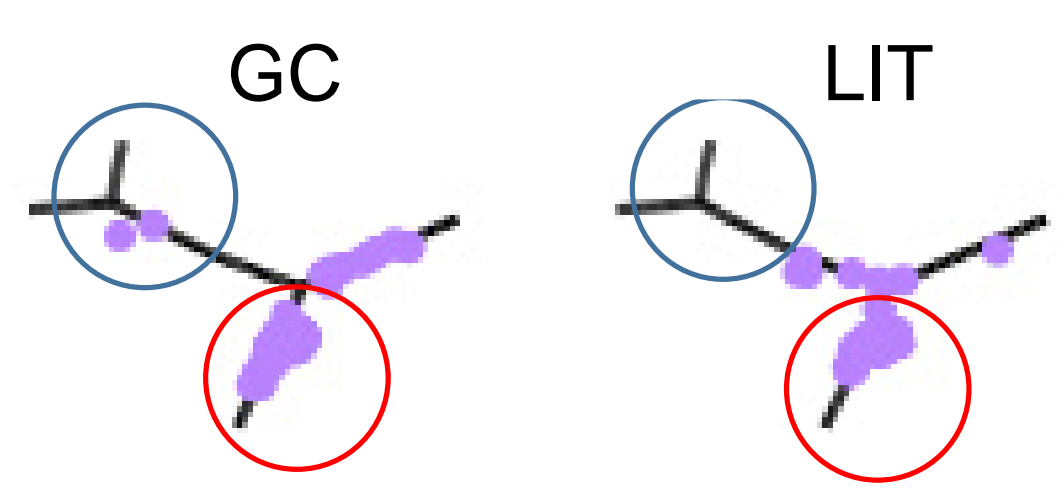

\section{C7:M1-like Macrophage}

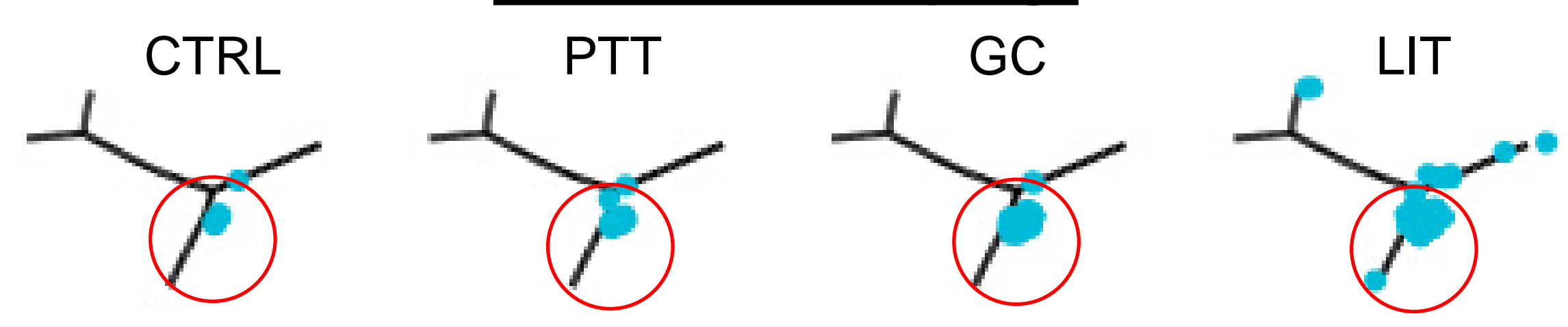

\section{C14:Monocyte derived cDC}

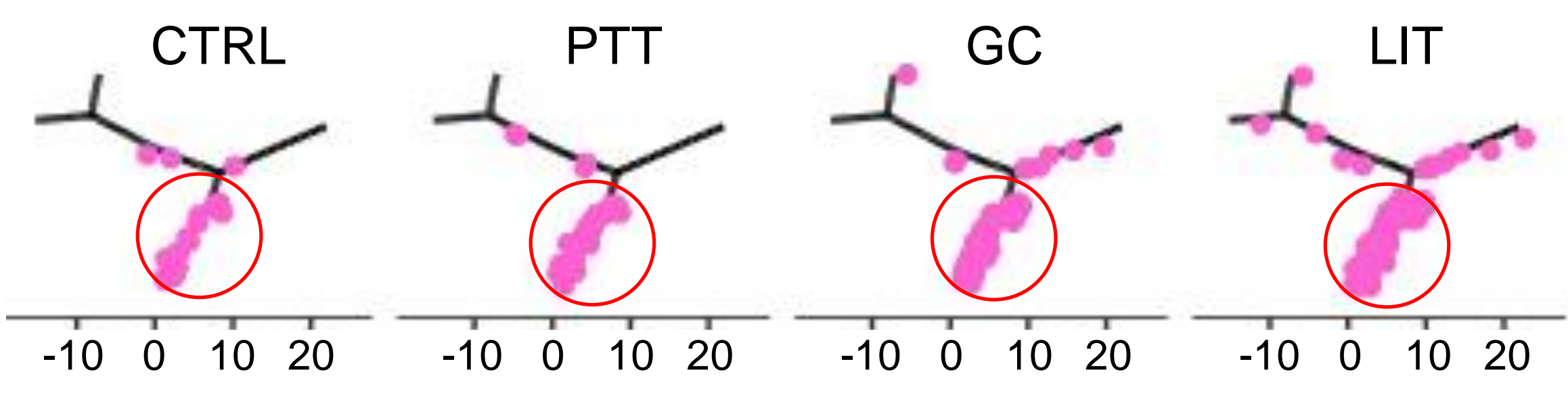

Figure 6. 